\title{
From Newton's method to exotic basins Part II: Bifurcation of the Mandelbrot-like sets
}

\author{
by \\ Krzysztof Barański (Warszawa)
}

\begin{abstract}
This is a continuation of the work [Ba] dealing with the family of all cubic rational maps with two supersinks. We prove the existence of the following parabolic bifurcation of Mandelbrot-like sets in the parameter space of this family. Starting from a Mandelbrot-like set in cubic Newton maps and changing parameters in a continuous way, we construct a path of Mandelbrot-like sets ending in the family of parabolic maps with a fixed point of multiplier 1. Then it bifurcates into two paths of Mandelbrot-like sets, contained respectively in the set of maps with exotic or non-exotic basins. The non-exotic path ends at a Mandelbrot-like set in cubic polynomials.
\end{abstract}

1. Introduction. This paper extends the work started in $[\mathrm{Ba}]$. We study the family $\mathcal{F}=\left\{f_{a, b}\right\}$, where

$$
f_{a, b}(z)=a z^{2} \frac{b z+1-2 b}{(2-b) z-1}, \quad a \in \mathbb{C} \backslash\{0\}, b \in \mathbb{C} \backslash\{0,1\} .
$$

This family consists of cubic rational maps with two supersinks: 0 and $\infty$ and a critical point at 1 . As one can easily check,

$$
\begin{aligned}
& \text { the critical points of } f_{a, b} \text { are } 0, \infty, 1, u=u_{a, b}=\frac{2 b-1}{b(2-b)} \\
& f_{a, b}(0)=0, f_{a, b}(\infty)=\infty, f_{a, b}(1)=a, f_{a, b}(u)=\frac{a(2 b-1)^{3}}{b(2-b)^{3}} .
\end{aligned}
$$

The paper [Ba] contains a general description of $\mathcal{F}$ and its moduli space together with a detailed study of the subfamily $\mathcal{F}_{2} \subset \mathcal{F}$ consisting of maps for which the critical point 1 is periodic of period 2 . In particular, a parabolic bifurcation in $\mathcal{F}_{2}$ from a Newton map to maps with exotic and non-exotic basins is described ([Ba], Theorem 4.20). This theorem says that there exists

2000 Mathematics Subject Classification: Primary 37F45.

Research supported by Polish KBN Grants No 2 P301 01307 and 2 P03A 025 12, Foundation for Polish Science and the Centre de Recerca Matemàtica in Bellaterra. 
a curve in the parameter plane of $\mathcal{F}_{2}$ joining a Newton map $N \in \mathcal{F}_{2}$ via maps with an attracting fixed point to a parabolic map $P \in \mathcal{F}_{2}$ with a fixed point of multiplier 1 . Then this curve bifurcates to two curves starting at $P$, contained respectively in the set of maps with exotic or non-exotic basins. Recall that an exotic basin (a notion introduced by F. Przytycki [P1]) is a non-simply connected completely invariant basin of an attracting fixed point, containing $k$ critical points counted with multiplicity, with $k$ less than the degree of the map. (For more information we refer to [Ba].)

This paper generalizes this result, proving the existence of a parabolic bifurcation of Mandelbrot-like sets in $\mathcal{F}$ (Theorem 4.1). It is known (see [CGS], [DH2]) that in the parameter plane of cubic Newton maps $\left\{N_{\sigma}\right\}_{\sigma \in \mathbb{C}}$ there exist Mandelbrot-like sets quasiconformally homeomorphic to the standard Mandelbrot set. These sets correspond to some regions of parameters $\sigma$ for which $N_{\sigma}^{k}$ is quadratic-like on some topological disc $U_{\sigma}$. Given such a Mandelbrot-like set $M_{N}$, we prove the existence of a continuous path of Mandelbrot-like sets joining $M_{N}$ via $M_{t}, t \in(0,1)$, contained in the family of maps with an attracting fixed point of multiplier $t$ to a parabolic $M_{1}$ contained in the family of maps with a fixed point of multiplier 1 . Then this path bifurcates into two paths of Mandelbrot-like sets, contained in the set of maps with exotic or non-exotic basins (see Figs. 1 and 2-5). Moreover, the non-exotic path can be extended in such a way that it terminates with a Mandelbrot-like set in the family of maps conformally conjugate to cubic polynomials with a supersink. Theorem 4.1 answers a question asked by F. Przytycki [P1] (see [Ba], Section 1).

The plan of the paper is as follows. In Section 2 we prove some technical lemmas which are used in the proof of the main theorem. They concern the limit behaviour of some invariant curves under the action of Riemann mappings. Section 3 contains definitions and properties of Mandelbrot-like families taken from [DH2]. The formulation and the proof of the main theorem (Theorem 4.1) are contained in Section 4. The proof, quite long and technical, is divided into several parts. In Subsection 4.1 we modify the initial Mandelbrot-like family in Newton maps to an equivalent family which has a nice combinatorial description. Then in Subsection 4.2 we construct the sets $M_{t}$ for $t \in(0,1)$ using quasiconformal surgery. The most delicate part is to prove that $M_{t}$ tends to a parabolic $M_{1}$ for $t \rightarrow 1^{-}$. This is done in Subsection 4.3. (The problem of obtaining a parabolic map as a limit of hyperbolic maps was studied in [Ma]. However, his theory is not applicable to our case.) In Subsection 4.4 we describe the bifurcation of $M_{1}$ into two paths by the use of the "tour de valse" method from [DH1]. In Subsection 4.5 we determine which path is exotic and in Subsection 4.6 we extend the non-exotic path in such a way that it ends in maps with a double supersink (i.e. conformally conjugate to cubic polynomials). 
Notation. The symbols cl, $\partial$ and int denote respectively the closure, boundary and interior of a set, $\widehat{\mathbb{C}}=\mathbb{C} \cup\{\infty\}$ is the Riemann sphere, $\mathbb{D}$ is the open unit disc in $\mathbb{C}, \mathbb{D}_{r}$ is the open disc centred at 0 of radius $r$ and $\mathbb{D}_{r}(x)$ is the open disc centred at $x$ of radius $r$. Define also $\mathbb{D}^{+}=\{z \in \mathbb{D}: \operatorname{Im}(z)>0\}$, $\mathbb{D}^{-}=\{z \in \mathbb{D}: \operatorname{Im}(z)<0\}$. By a simple arc we mean a curve homeomorphic to a line segment, and a topological disc is a set homeomorphic to $\mathbb{D}$. If $\gamma:[0, \infty) \rightarrow \widehat{\mathbb{C}}$ is a curve such that $\gamma(s) \rightarrow z$ as $s \rightarrow \infty$, then we say that $\gamma$ begins at $\gamma(0)$ and lands at $z$. If $\zeta$ is an attracting (resp. parabolic) point for a map $f$, then $B(\zeta)$ denotes the immediate basin of attraction to $\zeta$ (resp. invariant parabolic basin of $\zeta$ ).

REMARK. We often refer to the notions and results from [Ba], so it is advisable to read the two papers together. We also adopt some notational conventions from the first part of the work. Referring to [Ba], we write Theorem I.N.M for Theorem N.M of [Ba] etc.

Acknowledgements. The author would like to thank Prof. F. Przytycki for suggesting the problem and many fruitful discussions on the subject. $\mathrm{He}$ is grateful to the referee for carefully reading the manuscript, pointing out mistakes and proposing many improvements. He also thanks the Centre de Recerca Matemàtica in Bellaterra for support and hospitality.

\section{Convergence of Riemann mappings and invariant curves}

Definition 2.1. Denote by Sing the set of singular parameters $(a, b) \in$ $\widehat{\mathbb{C}} \times \widehat{\mathbb{C}}$ for the family $\mathcal{F}$, i.e.

$$
\text { Sing }=\{(a, b) \in \widehat{\mathbb{C}} \times \widehat{\mathbb{C}}: a \in\{0, \infty\} \text { or } b \in\{0,1, \infty\}\} .
$$

For $\varrho \in \mathbb{C}$ let $\operatorname{Fix}(\varrho)$ be the set of parameters $(a, b) \in(\widehat{\mathbb{C}} \times \widehat{\mathbb{C}}) \backslash$ Sing for which $f_{a, b} \in \mathcal{F}$ has a fixed point $\xi \neq 0, \infty$ of multiplier $\varrho$.

REMARK. There are four fixed points of $f_{a, b}$ (counted with multiplicity): $0, \infty$ and two others; denote them by $\xi_{1}, \xi_{2}$. Let $\varrho_{i}=f_{a, b}^{\prime}\left(\xi_{i}\right)$. By the holomorphic fixed point formula (see e.g. [Mi]), if $\varrho_{i} \neq 1$, then

$$
\frac{1}{1-\varrho_{1}}+\frac{1}{1-\varrho_{2}}=-1
$$

In other words, the value of one multiplier determines the other one and

$$
\operatorname{Fix}(\varrho)=\operatorname{Fix}\left(2+\frac{1}{\varrho-2}\right) .
$$

In this section we prove two technical lemmas, which will be used in the proof of the main theorem. The first one describes some standard facts on convergence of maps from $\mathcal{F}$ and suitable Riemann mappings. Recall first the definition of the convergence in the Carathéodory topology (see e.g. $[\mathrm{McM}])$. 
DeFinition 2.2. Let $\left(U_{n}, z_{n}\right), z_{n} \in U_{n} \subset \widehat{\mathbb{C}}$, be a sequence of pointed topological discs. We say that $\left(U_{n}, z_{n}\right)$ tends to a pointed topological disc $(U, z), z \in U \subset \widehat{\mathbb{C}}$, in the Carathéodory topology if $z_{n} \rightarrow z$, for every compact set $K \subset U$ we have $K \subset U_{n}$ for sufficiently large $n$ and for every open connected set $V$ containing $z$, if $V \subset U_{n}$ for infinitely many $n$, then $V \subset U$.

Lemma 2.3. Let $f_{n}=f_{a_{n}, b_{n}} \in \mathcal{F}$ such that $\left(a_{n}, b_{n}\right) \rightarrow(a, b)$ as $n \rightarrow \infty$ for some $a, b \in \widehat{\mathbb{C}}, a \neq 0, \infty$. Suppose that for every $n$ the map $f_{n}$ has a fixed point $\xi_{n} \neq 0, \infty$, either attracting with the immediate basin $B\left(\xi_{n}\right)$ or parabolic with an invariant parabolic basin $B\left(\xi_{n}\right)$. Let $\zeta_{n}$ be one of the points $0, \infty, \xi_{n}$ (the same choice for all $n$ ) and let $c_{n}$ be an $f_{n}$-critical point such that $c_{n} \in B\left(\zeta_{n}\right), c_{n} \rightarrow c \in \widehat{\mathbb{C}}$. Assume also that there exists $r>0$ such that for all $n$ the basin $B\left(\zeta_{n}\right)$ contains a disc of spherical radius $r$ centred at $c_{n}$ and $\widehat{\mathbb{C}} \backslash B\left(\zeta_{n}\right)$ contains a non-empty open set independent of $n$.

Then $B\left(\zeta_{n}\right)$ is simply connected for every $n$, the map $f=f_{a, b}$ has a fixed point $\zeta \in \widehat{\mathbb{C}}$, either attracting with the immediate basin $B(\zeta)$ or parabolic of multiplier 1 with exactly one invariant parabolic basin $B(\zeta)$ such that in both cases $B(\zeta)$ is simply connected, $c \in B(\zeta)$ and $\left(B\left(\zeta_{n}\right), c_{n}\right)$ tends to $(B(\zeta), c)$ in the Carathéodory topology. The sequence $\zeta_{n}$ does not always converge to $\zeta$, but if $\zeta_{n} \nrightarrow \zeta$, then $\zeta$ is parabolic and $\zeta_{n} \in\{0, \infty\}$ for infinitely many $n$.

Moreover, if $\psi_{n}: \mathbb{D} \rightarrow B\left(\zeta_{n}\right)$ is the unique Riemann mapping such that $\psi_{n}(0)=c_{n}$ and $\psi_{n}^{\prime}(0)>0$, then $\psi_{n} \rightarrow \psi$ almost uniformly on $\mathbb{D}$, where $\psi: \mathbb{D} \rightarrow B(\zeta)$ is the Riemann mapping such that $\psi(0)=c$ and $\psi^{\prime}(0)>0$. Furthermore, the Blaschke product $h_{n}=\psi_{n}^{-1} \circ f_{n} \circ \psi_{n}$ tends to $h=\psi^{-1} \circ f \circ \psi$ almost uniformly in the spherical metric on $\widehat{\mathbb{C}}$ outside at most one point $z_{0} \in \partial \mathbb{D}$.

REMARK. We allow here $(a, b)$ to be a singular parameter, which means that $f_{a, b}$ can be a map of degree two.

Proof. Observe first that by the Flower Theorem, if there exists an invariant parabolic basin, then the multiplier of the fixed point is equal to 1 . Moreover, if $f_{n}$ has an attracting fixed point, then by (3), the fourth fixed point is repelling and if $f_{n}$ has a parabolic fixed point of multiplier 1 , then there are only three fixed points. According to [Sh], if for a rational map only one fixed point is repelling or parabolic of multiplier 1, then each Fatou component is simply connected. Hence, $B\left(\zeta_{n}\right)$ is simply connected.

By assumption, $a \notin\{0, \infty\}$, so if $b \notin\{0,1, \infty\}$, then $(a, b) \notin$ Sing and $f_{n} \rightarrow f$ uniformly in the spherical metric on $\widehat{\mathbb{C}}$. Moreover, by Lemma I.2.2, if $b \in\{0,1, \infty\}$, then $f_{n} \rightarrow f$ almost uniformly in the spherical metric on $\widehat{\mathbb{C}} \backslash\{1 / b\}$ (with the convention $1 / 0=\infty, 1 / \infty=0$ ). Hence, we get:

(4) If $b \notin\{0,1, \infty\}$ or $z \in \widehat{\mathbb{C}} \backslash\{1 / b\}$, then $f_{n} \rightarrow f$ uniformly on $V_{z}$ 
for some neighbourhood $V_{z} \subset \widehat{\mathbb{C}}$ of $z$. Moreover, using Lemma I.2.2, it is easy to check that

If $b \in\{0,1, \infty\}$, then $\operatorname{dist}\left(1 / b, J\left(f_{n}\right)\right) \rightarrow 0$,

where $J\left(f_{n}\right)$ is the Julia set of $f_{n}$ and dist denotes the spherical distance.

By assumption, $\widehat{\mathbb{C}} \backslash B\left(\zeta_{n}\right)$ contains a non-empty open set independent of $n$, so the family $\left\{\psi_{n}\right\}_{n>0}$ is normal and $\psi_{n_{k}} \rightarrow \psi$ as $k \rightarrow \infty$ almost uniformly on $\mathbb{D}$ for some holomorphic map $\psi: \mathbb{D} \rightarrow \mathbb{C}$. Moreover, $\psi_{n_{k}}(\mathbb{D})$ contains the disc of spherical radius $r / 2$ centred at $c$ for large $k$. Hence, $\psi$ is univalent.

Note that $h_{n}$ is a quadratic or cubic Blaschke product with an attracting or parabolic fixed point and a critical point at 0. Passing to a subsequence, we can assume that $h_{n_{k}} \rightarrow h$ almost uniformly on $\mathbb{D}$ for some holomorphic map $h$, which is a Blaschke product of degree at most three or a constant. By definition, for $z \in \mathbb{D}$ we have

$$
f_{n_{k}}\left(\psi_{n_{k}}(z)\right)=\psi_{n_{k}}\left(h_{n_{k}}(z)\right) .
$$

Fix $z \in \mathbb{D}$. Then $\psi_{n_{k}}\left(h_{n_{k}}(z)\right) \rightarrow \psi(h(z))$. Moreover, $f_{n_{k}}\left(\psi_{n_{k}}(z)\right) \rightarrow f(\psi(z))$, provided $f_{n_{k}} \rightarrow f$ uniformly in a neighbourhood of $\psi(z)$. By (4), this holds if $b \notin\{0,1, \infty\}$ or $\psi(z)=1 / b$. Suppose now $b \in\{0,1, \infty\}, \psi(z)=1 / b$. Then $1 / b \in \psi(\mathbb{D})$, so by the almost uniform convergence $\psi_{n_{k}} \rightarrow \psi$, there exists a neighbourhood of $1 / b$ contained in $B\left(\zeta_{n_{k}}\right)$ for sufficiently large $k$. This contradicts (5), which shows that this case is impossible. We conclude that we can pass to the limit in (6), obtaining

$$
f \circ \psi=\psi \circ h,
$$

which means that $f$ on $\psi(\mathbb{D})$ is conformally conjugate to $h$ on $\mathbb{D}$. Note that since $\psi$ is univalent, $h$ is not constant and has a critical point at 0 , so it is a quadratic or cubic Blaschke product. Moreover, $f(\psi(\mathbb{D})) \subset \psi(\mathbb{D})$, so $\psi(\mathbb{D})$ is contained in an invariant Fatou component $F$. It cannot be a rotation domain, since it contains a critical point. Hence, either $F$ is the immediate basin $B(\zeta)$ of an attracting fixed point $\zeta$ or $F$ is an invariant parabolic basin $B(\zeta)$ for a parabolic fixed point $\zeta$ (by the Flower Theorem, in this case the multiplier must be equal to 1$)$. To prove that in the latter case $B(\zeta)$ is the unique invariant parabolic basin of $\zeta$, we show that for all maps $f_{a, b}$ for $a, b \in \widehat{\mathbb{C}}$, if there exists a fixed point of multiplier 1 , then it has only one invariant basin. This is obvious for $(a, b) \in$ Sing. To check the case $(a, b) \notin$ Sing, change conformally the coordinates fixing $0, \infty$ and sending the parabolic point to 1 . It is easy to check that such a map in these coordinates has the form

$$
\widetilde{f}(z)=z^{2} \frac{z+\beta-2}{\beta z-1}, \quad \beta \in \mathbb{C} \backslash\{1\},
$$


and $\tilde{f}(1)=\widetilde{f}^{\prime}(1)=1, \tilde{f}^{\prime \prime}(1)=2 /(b-1) \neq 0$. By the Flower Theorem, the fixed point of multiplier 1 has only one petal. Hence, $B(\zeta)$ is unique.

Now we return to the general case. By (7) and the dynamics of $f$, it is easy to check that no point $z \in \partial \psi(\mathbb{D})$ can be in $B(\zeta)$, so in fact we have $\psi(\mathbb{D})=B(\zeta)$. Hence, $B(\zeta)$ is simply connected and contains the critical point $c=\psi(0)$. It is clear that $\psi$ is the unique Riemann mapping from $\mathbb{D}$ onto $B(\zeta)$ such that $\psi(0)=c$ and $\psi^{\prime}(0)>0$.

By normality, for any subsequence $n_{j}$ we can choose a subsubsequence $n_{j_{k}}$ and repeat the above arguments, showing that $\psi_{n_{j_{k}}} \rightarrow \psi$ and $h_{n_{j_{k}}} \rightarrow h$ as $k \rightarrow \infty$ for the same $\psi, h$. It follows that in fact $\psi_{n}$ converges to $\psi$ and $h_{n}$ converges to $h$ almost uniformly on $\mathbb{D}$. Then the sequence of pointed topological discs $\left(\psi_{n}(\mathbb{D}), \psi_{n}(0)\right)=\left(B\left(\zeta_{n}\right), c_{n}\right)$ tends to $(\psi(\mathbb{D}), \psi(0))=(B(\zeta), c)$ in the Carathéodory topology (see e.g. $[\mathrm{McM}])$.

Now we check when $\zeta_{n} \rightarrow \zeta$. Suppose first that $\zeta$ is attracting. Then by the Carathéodory convergence of $\left(B\left(\zeta_{n}\right), c_{n}\right)$ to $(B(\zeta), c)$ and by $(5)$, we have $b \notin\{0,1, \infty\}$ or $\zeta \neq 1 / b$. Hence, by $(4), f_{n} \rightarrow f$ uniformly in some neighbourhood of $\zeta$, which easily implies that for large $n$ there exists an attracting $f_{n}$-fixed point $\zeta_{n}^{\prime}$ such that $\zeta_{n}^{\prime} \rightarrow \zeta$. But by $(3)$, there can be at most one attracting fixed point for $f_{n}$, so $\zeta_{n}=\zeta_{n}^{\prime} \rightarrow \zeta$.

Assume now that $\zeta$ is parabolic. As previously, if $b \notin\{0,1, \infty\}$ or $\zeta \neq 1 / b$, then $f_{n} \rightarrow f$ uniformly in a neighbourhood of $\zeta$. Hence, in this case there exist $f_{n}$-fixed points $\zeta_{n}^{\prime}, \zeta_{n}^{\prime \prime}$ such that $\zeta_{n}^{\prime}, \zeta_{n}^{\prime \prime} \rightarrow \zeta$ and $\zeta_{n}^{\prime}=\zeta_{n}^{\prime \prime}$ if and only if they have multiplier 1 . But since $f_{n}$ has only four (resp. three) fixed points in the attracting (resp. parabolic case), it follows that $\zeta_{n} \in\left\{\zeta_{n}^{\prime}, \zeta_{n}^{\prime \prime}, 0, \infty\right\}$. Hence, if $\zeta_{n} \nrightarrow \zeta$, then $\zeta_{n}=0$ or $\infty$ for infinitely many $n$. We are left with the case $b \in\{0,1, \infty\}, \zeta=1 / b$. Suppose $\zeta_{n} \nrightarrow \zeta$ and take a convergent subsequence $\zeta_{n_{j}} \rightarrow \widetilde{\zeta} \neq \zeta$. By (4), $f_{n} \rightarrow f$ uniformly in some neighbourhood of $\widetilde{\zeta}$. Therefore, $\widetilde{\zeta}$ is an $f$-fixed point, so it must be 0 or $\infty$. Moreover, by the uniform convergence $f_{n} \rightarrow f$ near $\widetilde{\zeta}$, we have $\zeta_{n_{j}} \in B(\widetilde{\zeta})$ for the map $f_{n_{j}}$ with large $j$, so $\zeta_{n_{j}}=\widetilde{\zeta}$ for large $j$. Hence, $\zeta_{n}=0$ or $\infty$ for infinitely many $n$.

It is clear that since $h_{n} \rightarrow h$ and $h_{n}, h$ are quadratic or cubic Blaschke products, the convergence is uniform in the spherical metric on $\widehat{\mathbb{C}}$ unless $\operatorname{deg} h_{n}=3$ and $\operatorname{deg} h=2$. In the latter case,

$$
h_{n}(z)=e^{i \theta_{n}} \frac{z-\alpha_{n}}{1-\bar{\alpha}_{n} z} \cdot \frac{z-\beta_{n}}{1-\bar{\beta}_{n} z} \cdot \frac{z-\gamma_{n}}{1-\bar{\gamma}_{n} z}
$$

with $\alpha_{n} \rightarrow z_{0} \in \partial \mathbb{D}, \beta_{n} \rightarrow z_{1} \in \mathbb{D}, \gamma_{n} \rightarrow z_{2} \in \mathbb{D}$ and it is easy to check that

$$
\frac{z-\alpha_{n}}{1-\bar{\alpha}_{n} z} \rightarrow-z_{0}
$$

almost uniformly in the spherical metric on $\widehat{\mathbb{C}} \backslash\left\{z_{0}\right\}$. This easily implies that $h_{n} \rightarrow h$ almost uniformly in the spherical metric on $\widehat{\mathbb{C}} \backslash\left\{z_{0}\right\}$. 
The second lemma asserts that in the situation of Lemma 2.3, a suitable converging sequence of $f_{n}$-invariant curves converges in the Hausdorff metric to a suitable $f$-invariant curve. We consider two cases. In the case (a) we have a sequence of backward $f_{n}$-invariant curves landing at a repelling or parabolic point and in the case (b) we consider a sequence of forward $f_{n^{-}}$ invariant curves containing an attracting point, landing at a repelling point and converging to a forward $f$-invariant curve landing at a parabolic point. The advantage of the lemma is that we do not assume that the sequence $f_{n}$ is uniformly convergent in a neighbourhood of the fixed point of $f$.

LEMmA 2.4. Under the assumptions of the previous lemma, assume additionally that for every $n$ the basin $B\left(\xi_{n}\right)$ contains a disc centred at an $f_{n}$-critical point of radius independent of $n$. Let $\gamma_{n}, \gamma$ be simple arcs in $\mathbb{D}$ parameterized by $s \in[0, \infty)$ such that $\gamma_{n}(s) \rightarrow \gamma(s)$ as $n \rightarrow \infty$ almost uniformly on $[0, \infty)$. Suppose one of the following two possibilities is satisfied:

(a) $h_{n}\left(\gamma_{n}(s)\right)=\gamma_{n}(s-1)$ for $s \geq 1$ and $\gamma_{n}$ (resp. $\left.\gamma\right)$ is disjoint from the closure of the postcritical set for $h_{n}$ (resp. $\left.h\right)$.

(b) $h_{n}\left(\gamma_{n}(s)\right)=\gamma_{n}(s+(n-s) / n)$ for $n>1, s \geq 0, \gamma_{n}((n, \infty))$ is disjoint from the closure of the $h_{n}$-postcritical set and diam $\gamma_{n}([n, \infty)) \rightarrow 0$.

Then $\gamma_{n}$ (resp. $\gamma$ ) lands at some $h_{n}$-fixed point $q_{n}$ (resp. $h$-fixed point $\left.q\right)$ in $\partial \mathbb{D}$ and $\psi_{n} \circ \gamma_{n}(r e s p . \psi \circ \gamma)$ lands at some $f_{n}$-fixed point $p_{n}$ (resp. $f$-fixed point $p)$ in $\widehat{\mathbb{C}}$. In the case (a) the points $q_{n}, p_{n}, q, p$ are either repelling or parabolic of multiplier 1 and in the case (b) the points $q_{n}, p_{n}$ are repelling and $q, p$ are parabolic of multiplier 1 .

Moreover, in both cases, for any sequences $n_{k}, s_{k}$,

$$
\text { if } n_{k}, s_{k} \underset{k \rightarrow \infty}{\longrightarrow} \infty \text {, then } \gamma_{n_{k}}\left(s_{k}\right) \underset{k \rightarrow \infty}{\longrightarrow} q \text { and } \psi_{n_{k}}\left(\gamma_{n_{k}}\left(s_{k}\right)\right) \underset{k \rightarrow \infty}{\longrightarrow} p \text {. }
$$

This implies that $q_{n} \rightarrow q, p_{n} \rightarrow p$ and $\gamma_{n} \cup\left\{q_{n}\right\}$ (resp. $\left.\psi_{n}\left(\gamma_{n}\right) \cup\left\{p_{n}\right\}\right)$ tends to $\gamma \cup\{q\}$ (resp. $\psi(\gamma) \cup\{p\})$ in the Hausdorff metric as compact subsets of the Riemann sphere.

Proof. The case (a). It is a general fact (the Landing Theorem) that under the assumptions of the lemma, $\gamma_{n}$ (resp. $\psi_{n} \circ \gamma_{n}$ ) lands at an $h_{n^{-}}$ fixed point $q_{n} \in \partial \mathbb{D}$ (resp. $f_{n}$-fixed point $p_{n} \in \widehat{\mathbb{C}}$ ), repelling or parabolic of multiplier 1 (see [TY]). Since $\gamma_{n}(s) \rightarrow \gamma(s)$ almost uniformly on $[0, \infty)$, passing to the limit we obtain

$$
h(\gamma(s))=\gamma(s-1), \quad f(\psi(\gamma(s)))=\psi(h(\gamma(s)))=\psi(\gamma(s-1)),
$$

so the Landing Theorem implies that $\gamma$ lands at an $h$-fixed point $q \in \partial \mathbb{D}$ and $\psi(\gamma)$ lands at an $f$-fixed point $p \in \widehat{\mathbb{C}}$.

Proof of $\gamma_{n_{k}}\left(s_{k}\right) \rightarrow q$. Let $n_{k}, s_{k} \rightarrow \infty$ and suppose $\gamma_{n_{k}}\left(s_{k}\right) \not \rightarrow$. Passing to a subsequence, we can assume $\gamma_{n_{k}}\left(s_{k}\right) \notin D$, where $D=\mathbb{D}_{\varepsilon}(q)$ for 
a small $\varepsilon>0$. By Lemma 2.3, $h_{n} \rightarrow h$ almost uniformly in the spherical metric on $\widehat{\mathbb{C}}$ outside at most one point in $\partial \mathbb{D}$. Hence, changing $\varepsilon$ if necessary, we can assume $h_{n} \rightarrow h$ uniformly on $\partial D$. Since $\gamma$ lands at $q$, we have $\gamma(s) \in \mathbb{D}_{\varepsilon / 2}(q)$ for large $s$, so by the almost uniform convergence $\gamma_{n} \rightarrow \gamma$, we can find $s_{0}>1$ such that for sufficiently large $k$,

$$
\gamma_{n_{k}}\left(\left[s_{0}-1, s_{0}\right]\right) \subset D \text {. }
$$

Let

$$
s_{k}^{\prime}=\inf \left\{s \in\left(s_{0}, s_{k}\right): \gamma_{n_{k}}(s) \in \partial D\right\} .
$$

Then (8) and (9) give

$$
h_{n_{k}}\left(\gamma_{n_{k}}\left(s_{k}^{\prime}\right)\right)=\gamma_{n_{k}}\left(s_{k}^{\prime}-1\right) \in D .
$$

If $q$ is repelling for $h$, then $h(\partial D) \cap \mathrm{cl} D=\emptyset$ (if we take $\varepsilon$ small enough), so by the uniform convergence $h_{n} \rightarrow h$ on $\partial D$, we have $h_{n_{k}}(\partial D) \cap \operatorname{cl} D=\emptyset$ for large $k$, which contradicts (10).

We are left with the case when $q$ is parabolic for $h$ of multiplier 1 . Then by the local study of a holomorphic map near such a point, the uniform convergence $h_{n} \rightarrow h$ on $\partial D$ and (10) we obtain

$$
\gamma_{n_{k}}\left(s_{k}^{\prime}\right) \in K \subset \mathbb{D},
$$

where $K$ is a compact set independent of $k$. Note that in the case when $q$ is parabolic we have $\zeta_{n}=\xi_{n}$, because if $\zeta_{n} \in\{0, \infty\}$, then $h_{n}$ (and hence $h$ ) would have a supersink at 0 , which is impossible, because $h$ is a Blaschke product and $\mathbb{D} \subset B(q)$.

By (11), $\gamma_{n_{k}}\left(s_{k}^{\prime}\right) \in \mathbb{D}_{r_{0}}$ for some $r_{0}<1$ independent of $k$. Since the segment $[0, q]$ forms the attracting axis of the parabolic point $q$, by the almost uniform convergence $h_{n} \rightarrow h$ on $\mathbb{D}$ we can find a positive integer $j$ and a small $\varepsilon_{1}>0$ such that for $s_{k}^{\prime \prime}=s_{k}^{\prime}-j>1$ we have

$$
\left|\gamma_{n_{k}}\left(s_{k}^{\prime \prime}\right)-q\right|<\varepsilon_{1} \quad \text { and } \operatorname{dist}\left(\gamma_{n_{k}}\left(s_{k}^{\prime \prime}\right),[0, q]\right)<\left|\gamma_{n_{k}}\left(s_{k}^{\prime \prime}\right)-q\right| / 10
$$

for large $k$.

Now we prove

$$
\psi_{n}^{-1}\left(\xi_{n}\right) \rightarrow q .
$$

To do it, suppose (13) does not hold and (passing to a subsequence) assume $\psi_{n}^{-1}\left(\xi_{n}\right) \rightarrow \widetilde{q} \neq q$. Recall that $h_{n} \rightarrow h$ almost uniformly in the spherical metric outside at most one point $z_{0} \in \partial \mathbb{D}$. If $\widetilde{q} \neq z_{0}$, then by the uniform convergence in a neighbourhood of $\widetilde{q}$, the map $h$ has two fixed non-repelling points $\widetilde{q} \neq q$ in $\mathrm{cl} \mathbb{D}$, which is clearly impossible for a quadratic or cubic Blaschke product. If $\widetilde{q}=z_{0}$, then by the uniform convergence in a neighbourhood of $q$, it is easy to check that for large $n$ we have two repelling $h_{n}$-fixed points $q^{\prime}, q^{\prime \prime} \in \partial \mathbb{D}$ in a small neighbourhood of $q$. But by uniform 
convergence, the small arc $\left(q^{\prime}, q^{\prime \prime}\right)$ in $\partial \mathbb{D}$ is $h_{n}$-invariant, so there exists another $h_{n}$-fixed point in this arc, which is impossible. This proves (13).

Due to (13), we can assume

$$
\left|\psi_{n_{k}}^{-1}\left(\xi_{n_{k}}\right)-q\right|<\varepsilon_{1} / 10 \text {. }
$$

Let $D_{k} \subset \mathbb{D}$ be the open hyperbolic disc (i.e. the disc with respect to the hyperbolic metric in $\mathbb{D}$ ) centred at $\psi_{n_{k}}^{-1}\left(\xi_{n_{k}}\right)$ such that $\gamma_{n_{k}}\left(s_{k}^{\prime \prime}\right) \in \partial D_{k}$ (in the case when $\psi_{n_{k}}^{-1}\left(\xi_{n_{k}}\right)$ is attracting) or the horodisc tangent to $\partial \mathbb{D}$ at $\psi_{n_{k}}^{-1}\left(\xi_{n_{k}}\right)$ such that $\gamma_{n_{k}}\left(s_{k}^{\prime \prime}\right) \in \partial D_{k}$ (in the case when $\psi_{n_{k}}^{-1}\left(\xi_{n_{k}}\right)$ is parabolic). Then in both cases we have

$$
\gamma_{n_{k}}\left(s_{k}^{\prime \prime}-1\right) \in D_{k} \quad \text { and } \quad h_{n_{k}}\left(D_{k}\right) \subset D_{k}
$$

(see e.g. [St]), so $D_{k}$ contains a point from $\gamma_{n_{k}}([0,1])$. By the almost uniform convergence $\gamma_{n} \rightarrow \gamma$, this point lies in some fixed compact set in $\mathbb{D}$. On the other hand, it is easy to check by elementary geometry that (12) and (14) imply the Euclidean diameter of $D_{k}$ is less than $2 \varepsilon_{1}$, so $D_{k} \subset \mathbb{D}_{3 \varepsilon_{1}}(q)$. For sufficiently small $\varepsilon_{1}$ we get a contradiction.

Proof of $\psi_{n_{k}}\left(\gamma_{n_{k}}\left(s_{k}\right)\right) \rightarrow p$. Define $\varepsilon, D, s_{k}, s_{0}, s_{k}^{\prime}$ as previously, replacing $h_{n_{k}}, h, q, \gamma_{n_{k}}$ respectively by $f_{n_{k}}, f, p, \psi_{n_{k}} \circ \gamma_{n_{k}}$. If $p$ is repelling, then the proof is the same. Consider the case when $p$ is parabolic. Then repeating the proof, instead of (11) we obtain

$$
\psi_{n_{k}}\left(\gamma_{n_{k}}\left(s_{k}^{\prime}\right)\right) \in K
$$

where $K$ is a compact set independent of $k$ contained in the unique $f$ invariant parabolic basin $B(p)$ of $p$ (recall that by Lemma $2.3, p$ has only one invariant basin). By assumption, $B\left(\xi_{n}\right)$ contains a disc of a fixed radius centred at some $f_{n}$-critical point $\widetilde{c}_{n}$. It is easy to see that $B(0) \cup B(\infty)$ also contains a disc of a spherical radius independent of $n$. Hence, the assumptions of Lemma 2.3 are fulfilled for $B\left(\xi_{n}\right)$, so $\left(B\left(\xi_{n}\right), \widetilde{c}_{n}\right)$ tends in the Carathéodory topology to $\left(\widetilde{B}(\zeta), \lim _{n} \widetilde{c}_{n}\right)$ for an $f$-invariant parabolic basin $\widetilde{B}(\zeta)$ of $\zeta$. It is clear that $\zeta=p$ and $\widetilde{B}(\zeta)=B(p)$. By the Carathéodory convergence, $K \subset B\left(\xi_{n_{k}}\right)$ for large $k$. Hence, $\zeta_{n_{k}}=\xi_{n_{k}}$ and $\psi(\mathbb{D})=B(p)$, so

$$
K \subset \psi(\mathbb{D}) .
$$

Thus, $\psi^{-1}(K) \subset \mathbb{D}_{r_{1}}$ for some $r_{1}<1$. By the uniform convergence $\psi_{n_{k}} \rightarrow \psi$ on $\mathbb{D}_{r_{1}}$, we have $K \subset \psi_{n_{k}}\left(\mathbb{D}_{r_{1}}\right)$ for large $k$. But by $(15), \psi_{n_{k}}^{-1}(K) \subset \mathbb{D}_{r_{1}}$ contains $\gamma_{n_{k}}\left(s_{k}^{\prime}\right)$, which contradicts $\gamma_{n_{k}}\left(s_{k}^{\prime}\right) \rightarrow q \in \partial \mathbb{D}$.

The case (b). Note first that the point $\gamma_{n}(n)$ is an attracting $h_{n}$-fixed point for every $n$. Hence, $\psi_{n}\left(\gamma_{n}(n)\right)=\xi_{n}=\zeta_{n}$ is an attracting $f_{n}$-fixed point. Moreover, the curve $\left.\gamma_{n}\right|_{[n+1, \infty)}$ fulfills (after rescaling) the conditions of the case (a). Therefore, $\gamma_{n}$ lands at an $h_{n}$-fixed point $q_{n} \in \partial \mathbb{D}$ and $\psi_{n}\left(\gamma_{n}\right)$ 
lands at an $f_{n}$-fixed point $p_{n} \in \widehat{\mathbb{C}}$. The points $q_{n}, p_{n}$ are repelling, since $h_{n}$ and $f_{n}$ have an attracting fixed point. By the almost uniform convergence $\gamma_{n}(s) \rightarrow \gamma(s)$, after passing to the limit we get

$$
h(\gamma(s))=\gamma(s+1), \quad f(\psi(\gamma(s)))=\psi(\gamma(s+1)) .
$$

By assumption, diam $\gamma_{n}([n, \infty)) \rightarrow 0$, so $\gamma_{n}(n) \rightarrow \partial \mathbb{D}$, which implies that $h$ (and hence $f$ ) must be parabolic. Thus, $\gamma(n)=h^{n}(\gamma(0))$ converges to a parabolic $h$-fixed point $q \in \partial \mathbb{D}$ of multiplier 1 and $\psi(\gamma(n))$ converges to a parabolic $f$-fixed point $p \in \widehat{\mathbb{C}}$ of multiplier 1 . Since the hyperbolic distance between any point from $\gamma$ and the set $\{\gamma(n)\}_{n>0}$ (resp. $\psi(\gamma)$ and $\left.\{\psi(\gamma(n))\}_{n>0}\right)$ is bounded by a fixed constant, we conclude that $\gamma$ lands at $q$ and $\psi(\gamma)$ lands at $p$.

Proof of $\gamma_{n_{k}}\left(s_{k}\right) \rightarrow q$. Note that since $q$ is parabolic, by (13) we have $\gamma_{n_{k}}\left(n_{k}\right)=\psi_{n_{k}}^{-1}\left(\xi_{n_{k}}\right) \rightarrow q$. Hence, using the assumption diam $\gamma_{n}([n, \infty)) \rightarrow 0$, we can assume $s_{k}<n_{k}$. Take a small $\varepsilon_{2}>0$. As $[0, q]$ forms the attracting axis of the parabolic point $q$, by the almost uniform convergence $h_{n} \rightarrow h$ we can find $s_{0}$ such that for every $s \in\left[s_{0}, s_{0}+1\right]$,

$$
\left|\gamma_{n_{k}}(s)-q\right|<\varepsilon_{2} \quad \text { and } \operatorname{dist}\left(\gamma_{n_{k}}(s),[0, q]\right)<\left|\gamma_{n_{k}}(s)-q\right| / 10
$$

for $k$ larger than some $k(s)$ (cf. (12)). As in the proof of the case (a), we find an open disc $D_{s, k}$ of Euclidean diameter less than $2 \varepsilon_{2}$ such that $h_{n_{k}}\left(D_{s, k}\right) \subset D_{s, k}$ and $\xi_{n_{k}}, \gamma_{n_{k}}(s) \in D_{s, k}$ for $k>k(s)$. By the compactness of $\left[s_{0}, s_{0}+1\right]$, there exists $k_{0}$ such that for every $k>k_{0}$ and every $s \geq s_{0}$ we have $\xi_{n_{k}}, \gamma_{n_{k}}(s) \in D_{s, k}$. Hence, $\left|\gamma_{n_{k}}(s)-\xi_{n_{k}}\right|<2 \varepsilon_{2}$, so $\left|\gamma_{n_{k}}(s)-q\right|<3 \varepsilon_{2}$ for every $s \geq s_{0}$. This proves $\gamma_{n_{k}}\left(s_{k}\right) \rightarrow q$.

Proof of $\psi_{n_{k}}\left(\gamma_{n_{k}}\left(s_{k}\right)\right) \rightarrow p$. Suppose this is not true and define $\varepsilon$ and $D$ as in the proof of the case (a). Note that $\psi_{n}\left(\gamma_{n}(n)\right)=\xi_{n} \rightarrow p$. (To see this, it is sufficient to notice that by Lemma I.2.2, $f_{n} \rightarrow f$ almost uniformly on $\widehat{\mathbb{C}} \backslash\{p\}$ and use similar arguments to those for (13).) Hence, $\psi_{n_{k}}\left(\gamma_{n_{k}}\left(n_{k}\right)\right) \in D$ for large $k$. Let

$$
s_{k}^{\prime}= \begin{cases}\sup \left\{s \in\left(s_{k}, n_{k}\right): \psi_{n_{k}}\left(\gamma_{n_{k}}(s)\right) \in \partial D\right\} & \text { if } s_{k}<n_{k}, \\ \inf \left\{s \in\left(n_{k}, s_{k}\right): \psi_{n_{k}}\left(\gamma_{n_{k}}(s)\right) \in \partial D\right\} & \text { if } s_{k}>n_{k} .\end{cases}
$$

Then $f_{n_{k}}\left(\psi_{n_{k}}\left(\gamma_{n_{k}}\left(s_{k}^{\prime}\right)\right)\right) \in D$. In the same way as in the case (a) we show that there exists a compact set $K$ and $r_{1}<1$ such that

$$
\psi_{n_{k}}\left(\gamma_{n_{k}}\left(s_{k}^{\prime}\right)\right) \in K \subset \psi_{n_{k}}\left(\mathbb{D}_{r_{1}}\right)
$$

for sufficiently large $k$. This contradicts $\gamma_{n_{k}}\left(s_{k}^{\prime}\right) \rightarrow q \in \partial \mathbb{D}$. Hence, $\psi_{n_{k}}\left(\gamma_{n_{k}}\left(s_{k}\right)\right) \rightarrow p$.

Note that the facts proven above easily imply $q_{n} \rightarrow q, p_{n} \rightarrow p$. Moreover, it is easy to see that they show (together with the almost uniform 
convergence $\left.\gamma_{n} \rightarrow \gamma, \psi_{n} \circ \gamma_{n} \rightarrow \psi \circ \gamma\right)$ that $\gamma_{n} \cup\left\{q_{n}\right\}$ tends to $\gamma \cup\{q\}$ and $\psi_{n}\left(\gamma_{n}\right) \cup\left\{p_{n}\right\}$ tends to $\psi(\gamma) \cup\{p\}$ in the Hausdorff metric.

3. Mandelbrot-like families. It is known (see [CGS], [DH2]) that in the parameter plane of cubic Newton maps $N$ there exist Mandelbrotlike sets (quasiconformally homeomorphic to the standard Mandelbrot set) corresponding to certain sets of parameters for which $\left.N^{k}\right|_{U}$ is quadraticlike on some topological disc $U$ with some $k \geq 2$. More precisely, consider the family $\mathcal{N}_{(0, u, \infty)} \subset \mathcal{F}$ of Newton maps with supersinks $0, u, \infty$. This family consists of maps $f_{a, b}$ with $a=\left(\frac{b-2}{2 b-1}\right)^{2}$ (see Section I.3). To avoid confusion in notation, we parameterize these maps by a new parameter $\sigma \in \Sigma=\mathbb{C} \backslash\{0,1 / 2,1,2\}$ setting

$$
N_{\sigma}=f_{a, b} \quad \text { for } \quad a=\left(\frac{\sigma-2}{2 \sigma-1}\right)^{2}, b=\sigma .
$$

Take $\sigma_{0}$ such that for $N_{\sigma_{0}}$ the critical point 1 is periodic with some period $k \geq 2$. According to Lemma I.4.19, $N_{\sigma_{0}}^{k}$ is quadratic-like on some topological disc $U_{\sigma_{0}}$ containing the critical point 1. Perturbing $\sigma$ within some open set $\mathcal{U} \subset \Sigma$ we obtain a family $\left\{N_{\sigma}\right\}_{\sigma \in \mathcal{U}}$ of maps such that $N_{\sigma}^{k}$ is quadratic-like on some topological disc $U_{\sigma}$ (see Subsection 4.1).

The general theory of such analytic families of polynomial-like maps was developed in [DH2]. For convenience, we recall here the definitions and some results from this work, which will be used in what follows.

Definition 3.1. A polynomial-like map of degree $d$ is a proper holomorphic mapping of degree $d$ from a topological disc $U \subset \widehat{\mathbb{C}}$ onto a topological disc $V \subset \widehat{\mathbb{C}}$ such that $\operatorname{cl} U \subset V$. The filled-in Julia set $K_{f}$ of $f$ is the set of points $z \in U$ such that the entire forward trajectory of $z$ is contained in $U$. A polynomial-like map of degree two is called a quadratic-like map.

Definition 3.2. Let $\left\{f_{\sigma}\right\}_{\sigma \in \mathcal{U}}$ for some complex manifold $\mathcal{U}$ be a family of quadratic-like maps $f_{\sigma}: U_{\sigma} \rightarrow f_{\sigma}\left(U_{\sigma}\right)$. Let $\mathcal{V}=\left\{(\sigma, z): z \in U_{\sigma}\right\}$, $\mathcal{W}=\left\{(\sigma, z): z \in f_{\sigma}\left(U_{\sigma}\right)\right\}, f(\sigma, z)=\left(\sigma, f_{\sigma}(z)\right)$. Then this family is an analytic family of quadratic-like maps if:

(i) $\mathcal{V}$ and $\mathcal{W}$ are homeomorphic over $\mathcal{U}$ to $\mathcal{U} \times \mathbb{D}$,

(ii) the projection $\operatorname{cl}_{\mathcal{W}} \mathcal{V} \rightarrow \mathcal{U}$ is a proper map,

(iii) $f: \mathcal{V} \rightarrow \mathcal{W}$ is a proper holomorphic map.

REMark. The condition (ii) of the above definition is fulfilled if $\operatorname{cl} U_{\sigma}$ depends continuously on $\sigma$ in the Hausdorff metric. Indeed, in this case for every $\sigma_{n} \in \mathcal{U}$ and $z_{n} \in \operatorname{cl} U_{\sigma_{n}}$, if $z_{n} \rightarrow z \in \widehat{\mathbb{C}}$ and $\sigma_{n} \rightarrow \sigma \in \mathcal{U}$, then $z \in U_{\sigma}$, which easily implies the condition (ii). 
The Straightening Theorem says that every quadratic-like map $f$ is hybrid equivalent to some polynomial $z^{2}+c$, i.e. $f$ is conjugate to $z^{2}+c$ by a quasiconformal homeomorphism $h$ which is conformal almost everywhere on the filled-in Julia set $K_{f}$ of $f$. Moreover, if $K_{f}$ is connected, then $c$ is uniquely determined. Hence, for a family $\left\{f_{\sigma}\right\}_{\sigma \in \mathcal{U}}$ we can define a function $\chi$ setting $\chi(\sigma)=c$ for $\sigma$ such that $K_{f_{\sigma}}$ is connected. Let $M$ be the standard Mandelbrot set for the family $z^{2}+c$. The following facts were proved in [DH2]:

THEOREM 3.3. For any analytic family $\left\{f_{\sigma}\right\}_{\sigma \in \mathcal{U}}$ of quadratic-like mappings, if $\mathcal{U}$ is a contractible complex manifold, then the mapping $\chi$ can be extended continuously to $\mathcal{U}$ and $\chi$ is holomorphic on $\operatorname{int} \chi^{-1}(M)$. Moreover, for every $c \in M$ the set $\chi^{-1}(c)$ is analytic. However, $\chi$ need not to be holomorphic at the boundary of $\chi^{-1}(M)$.

TheOREM 3.4. For any analytic family $\mathbf{f}=\left\{f_{\sigma}\right\}_{\sigma \in \mathcal{U}}$ of quadratic-like mappings with $\mathcal{U}$ connected of complex dimension one, if $\chi$ is not constant, then it is topologically holomorphic over $M$, i.e. for all $\sigma \in \chi^{-1}(M), \sigma$ is isolated in its fibre and the index of $\chi$ at $\sigma$ is strictly positive.

Proposition 3.5. Suppose that, in addition, $\mathcal{U}$ is a topological disc and $\chi^{-1}(M)$ is compact. Let $\omega_{\sigma}$ be the critical point of $f_{\sigma}$ and let $A$ be a closed topological disc in $\mathcal{U}$ such that $\chi^{-1}(M) \in \operatorname{int} A$. Then $\chi$ has degree $d$ on $\chi^{-1}(M)$, where $d$ is the number of times $f_{\sigma}\left(\omega_{\sigma}\right)-\omega_{\sigma}$ turns around 0 as $\sigma$ describes $\partial A$. In particular, if $d=1$, then $\chi$ is a homeomorphism on $\chi^{-1}(M)$ and we call the family $\left\{f_{\sigma}\right\}_{\sigma \in \mathcal{U}}$ a Mandelbrot-like family and $M_{\mathbf{f}}=$ $\chi^{-1}(M)$ a Mandelbrot-like set.

Definition 3.6. Let $\mathcal{U}$ be a Riemann surface homeomorphic to a disc and let

$$
\mathbf{f}=\left\{f_{\sigma, t}: U_{\sigma, t} \rightarrow f_{\sigma, t}\left(U_{\sigma, t}\right)\right\}_{(\sigma, t) \in \mathcal{U} \times[0,1]}
$$

be a family of quadratic-like maps. Suppose that the conditions (i) and (ii) from Definition 3.2 hold for $\mathcal{U}$ replaced by $\mathcal{U} \times[0,1]$ and the mapping $(\sigma, t, z) \mapsto\left(\sigma, t, f_{\sigma, t}(z)\right)$ is continuous and proper in $(\sigma, t, z)$ and holomorphic in $(\sigma, z)$. Assume also that for every $t \in[0,1]$ the family $\mathbf{f}_{t}=\left\{f_{\sigma, t}\right\}_{\sigma \in \mathcal{U}}$ is Mandelbrot-like and all sets $M_{\mathbf{f}_{t}}$ are contained in a common compact set $A \subset \mathcal{U}$. Then we say that $\mathbf{f}_{0}$ and $\mathbf{f}_{1}$ can be connected by a continuous path of Mandelbrot-like families.

Remark. As previously, the condition (ii) is satisfied if $\mathrm{cl} U_{\sigma, t}$ depends continuously on $\sigma, t$ in the Hausdorff metric.

Let $\mathcal{U}$ and $\mathcal{U}_{t}$ for $t \in[0,1]$ be disc-equivalent Riemann surfaces such that there exists a homeomorphism $\mathcal{H}: \mathcal{U} \times[0,1] \rightarrow \bigcup_{t \in[0,1]} \mathcal{U}_{t}$ which maps holomorphically $\mathcal{U} \times\{t\}$ onto $\mathcal{U}_{t}$ for every $t$. If $\left\{f_{\mathcal{H}(\sigma, t)}\right\}_{(\sigma, t) \in \mathcal{U} \times[0,1]}$ is a continuous 
path of Mandelbrot-like families, we will also say that $\left\{f_{\tau}\right\}_{\tau \in \mathcal{U}_{t}, t \in[0,1]}$ is a continuous path of Mandelbrot-like families.

Proposition 3.7. Let $\mathbf{f}=\left\{f_{\sigma}\right\}_{\sigma \in \mathcal{U}}$ and $\mathbf{g}=\left\{g_{\sigma}\right\}_{\sigma \in \mathcal{U}}$ be two Mandelbrotlike families parameterized by the same Riemann surface $\mathcal{U}$. If $\mathbf{f}$ and $\mathbf{g}$ can be connected by a continuous path of Mandelbrot-like families, then the homeomorphism $\chi_{\mathbf{g}}^{-1} \circ \chi_{\mathbf{f}}: M_{\mathbf{f}} \rightarrow M_{\mathbf{g}}$ is quasiconformal.

Moreover, [DH2] gives examples of topological $\operatorname{discs} \mathcal{U}$ in the parameter plane of cubic Newton maps such that (in our parameterization) $\mathcal{U} \subset \Sigma$, the family $\left\{\left.N_{\sigma}^{k}\right|_{U_{\sigma}}\right\}_{\sigma \in \mathcal{U}}$ is Mandelbrot-like for some topological discs $U_{\sigma}$ containing the critical point 1 and $\chi$ is quasiconformal on $\chi^{-1}(M)$.

Definition 3.8. We will say that two Mandelbrot-like families

$$
\mathbf{f}=\left\{f_{\sigma}: U_{\sigma} \rightarrow f_{\sigma}\left(U_{\sigma}\right)\right\}_{\sigma \in \mathcal{U}_{\mathbf{f}}} \text { and } \mathbf{g}=\left\{g_{\sigma}: V_{\sigma} \rightarrow g_{\sigma}\left(V_{\sigma}\right)\right\}_{\sigma \in \mathcal{U}_{\mathbf{g}}}
$$

with $\mathcal{U}_{\mathbf{f}}, \mathcal{U}_{\mathrm{g}} \subset \mathcal{U}$ for some Riemann surface $\mathcal{U}$ are equivalent if

(i) $M_{\mathbf{f}}=M_{\mathbf{g}} \subset \mathcal{U}_{\mathbf{f}} \cap \mathcal{U}_{\mathrm{g}}$,

(ii) $K_{f_{\sigma}}=K_{g_{\sigma}} \subset U_{\sigma} \cap V_{\sigma}$ and $\left.f_{\sigma}\right|_{U_{\sigma} \cap V_{\sigma}}=\left.g_{\sigma}\right|_{U_{\sigma} \cap V_{\sigma}}$ for every $\sigma \in \mathcal{U}_{\mathbf{f}} \cap \mathcal{U}_{\mathbf{g}}$.

4. The bifurcation theorem. In this section we formulate and prove the main theorem.

TheOREM 4.1 (The bifurcation theorem). Let $\mathbf{N}=\left\{\left.N_{\sigma}^{k}\right|_{U_{\sigma}}\right\}_{\sigma \in \mathcal{U}}$ for a topological disc $\mathcal{U} \subset \Sigma$ be a Mandelbrot-like family in $\mathcal{N}_{(0, u, \infty)}$ and let $M_{N}=\chi^{-1}(M)$ be the Mandelbrot-like set in $\mathcal{U}$. Set $\sigma_{0}=\chi^{-1}(0)$ and assume that for $N_{\sigma_{0}}$. Head's angle $\alpha$ is periodic mod 1 with period $k$. Then there exist continuous paths of Mandelbrot-like families $\left\{\mathbf{f}_{t}\right\}_{t \in[0,1]},\left\{\mathbf{f}_{t}^{0}\right\}_{t \in[1,1+\varepsilon]}$, $\left\{\mathbf{f}_{t}^{\infty}\right\}_{t \in[1,1+\varepsilon]}$ for some $0<\varepsilon<1$, with Mandelbrot-like sets $M_{t}, M_{t}^{0}, M_{t}^{\infty}$ respectively, such that:

- $\mathbf{f}_{0}=\left\{\left.N_{\sigma}^{k}\right|_{U_{\sigma}^{\prime}}\right\}_{\sigma \in \mathcal{U}^{\prime}}$ for some topological disc $\mathcal{U}^{\prime} \subset \Sigma$ and $\mathbf{f}_{0}$ is equivalent to $\mathbf{N}$, in particular $M_{0}=M_{N}$.

- For every $t \in(0,1)$ we have $\mathbf{f}_{t}=\left\{\left.f_{a, b}^{k}\right|_{U_{a, b}}\right\}_{(a, b) \in \mathcal{U}_{t}}$ for some topological disc $\mathcal{U}_{t} \subset \operatorname{Fix}(t)$ and for every $(a, b) \in \mathcal{U}_{t}$ the $f_{a, b}$-critical point $u_{a, b}$ is in the immediate basin of an attracting $f_{a, b}$-fixed point of multiplier $t$.

- $\mathbf{f}_{1}=\mathbf{f}_{1}^{0}=\mathbf{f}_{1}^{\infty}=\left\{\left.f_{a, b}^{k}\right|_{U_{a, b}}\right\}_{(a, b) \in \mathcal{U}_{1}}$ for some topological disc $\mathcal{U}_{1} \subset$ $\operatorname{Fix}(1)$ and for every $(a, b) \in \mathcal{U}_{1}$ the $f_{a, b}$-critical point $u_{a, b}$ is in the unique invariant parabolic basin of an $f_{a, b}$-fixed point of multiplier 1.

- For $\zeta \in\{0, \infty\}$ and every $t \in(1,1+\varepsilon]$ we have $\mathbf{f}_{t}^{\zeta}=\left\{\left.f_{a, b}^{k}\right|_{U_{a, b}}\right\}_{(a, b) \in \mathcal{U}_{t}^{\zeta}}$ for some topological disc $\mathcal{U}_{t}^{\zeta} \subset \widehat{\mathbb{C}} \times \widehat{\mathbb{C}} \backslash$ Sing such that $u_{a, b} \in B(\zeta)$ for every $(a, b) \in \mathcal{U}_{t}^{\zeta}$ and $\Phi_{a, b}^{\zeta}\left(f_{a, b}\left(u_{a, b}\right)\right)=2-t$ for every $(a, b) \in M_{t}^{\zeta}$, where $\Phi_{a, b}^{\zeta}$ denotes the Böttcher coordinates defined on the maximal subset of $B(\zeta)$ for the map $f_{a, b}$. 
- For $\zeta \in\{0, \infty\}$, if the set $\partial B(u) \cap \partial B(\zeta)$ for the map $N_{\sigma_{0}}$ in the initial family is infinite, then for $t \in(1,1+\varepsilon]$ the basin $B(\zeta)$ is not simply connected for every $(a, b) \in \mathcal{U}_{t}^{\zeta}$ and is exotic for every $(a, b) \in M_{t}^{\zeta}$; otherwise $B(\zeta)$ is simply connected for every $(a, b) \in M_{t}^{\zeta}$ and is not exotic for every $(a, b) \in \mathcal{U}_{t}^{\zeta}$.

- For $\zeta \in\{0, \infty\}$, if $B(\zeta)$ is not exotic for $(a, b) \in \mathcal{U}_{t}^{\zeta}, t \in(1,1+\varepsilon]$, then the path $\left\{\mathbf{f}_{t}^{\zeta}\right\}_{t \in[1,1+\varepsilon]}$ can be extended to a continuous path $\left\{\mathbf{f}_{t}^{\zeta}\right\}_{t \in[1,2]}$ for $\mathbf{f}_{t}^{\zeta}=\left\{\left.f_{a, b}^{k}\right|_{U_{a, b}}\right\}_{(a, b) \in \mathcal{U}_{t}^{\zeta}}$ such that for every $(a, b) \in \mathcal{U}_{t}^{\zeta}$ we have $u_{a, b} \in B(\zeta)$ for $t \in(1,2]$ and $u_{a, b}=\zeta$ for $t=2$. In particular, if $B(\infty)$ is not exotic for $(a, b) \in \mathcal{U}_{t}^{\infty}$, then the maps $f_{a, b}$ for $(a, b) \in \mathcal{U}_{2}^{\infty}$ are cubic polynomials with a supersink at 0 .

Moreover, if $\chi: M_{N} \rightarrow M$ is quasiconformal, then all the Mandelbrotlike sets $M_{t}, M_{t}^{0}, M_{t}^{\infty}$ are quasiconformally homeomorphic to $M$.

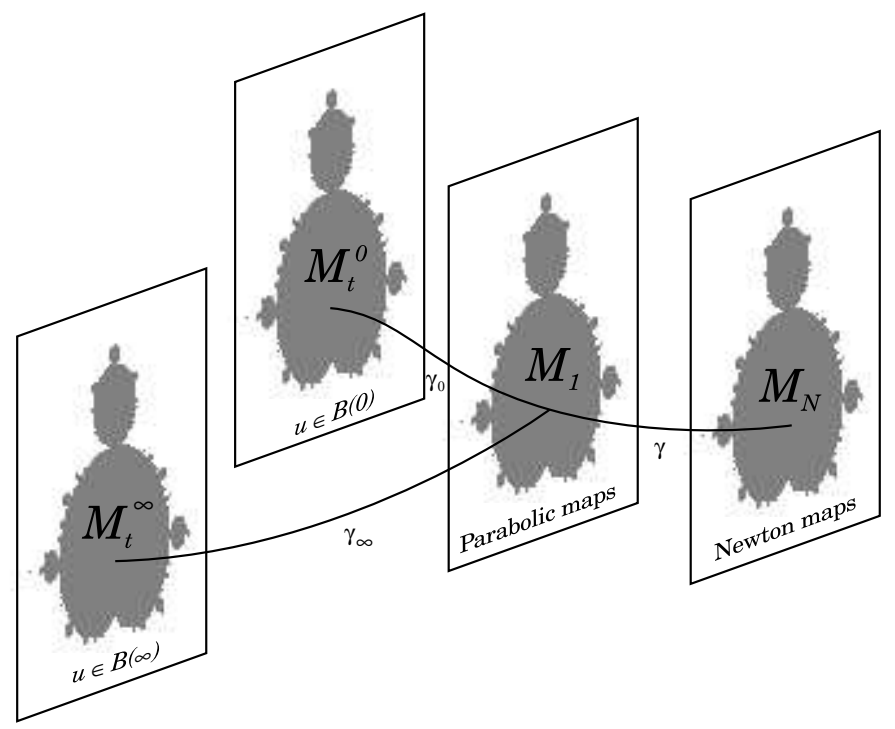

Fig. 1. Bifurcation of the Mandelbrot-like sets

Remark. It is not possible to extend the exotic path $\left\{\mathbf{f}_{t}^{\zeta}\right\}_{t \in[1,1+\varepsilon]}$ to $\left\{\mathbf{f}_{t}^{\zeta}\right\}_{t \in[1,2]}$ as in the non-exotic case, because for $t=2$ the map $f_{a, b}$ can degenerate (see Section I.2).

The parabolic bifurcation is demonstrated in Fig. 1, and Figs. 2-5 present computer pictures of the Mandelbrot-like sets. In all of them white (resp. dark grey) colour corresponds to parameters for which the critical point 1 is in the basin of $\infty$ (resp. 0). Note the remarkable similarity between the parameter space and the dynamical space in Fig. I.7. 


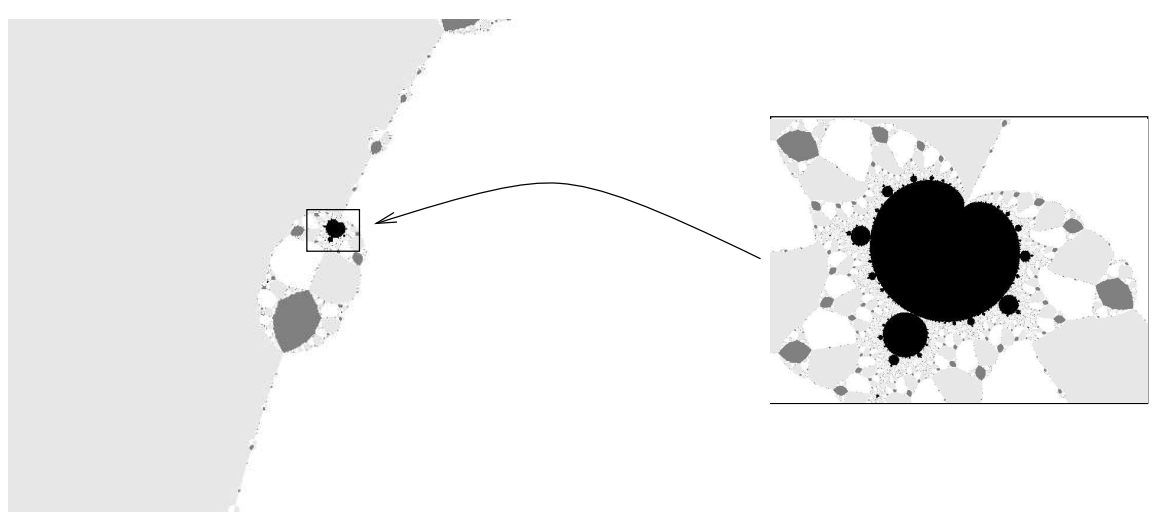

Fig. 2. A fragment of the parameter space of Newton maps with supersinks $0, \infty, u$. Light grey colour corresponds to parameters for which the critical point 1 is in the basin of $u$. The enlargement shows the Mandelbrot-like set $M_{N}$.
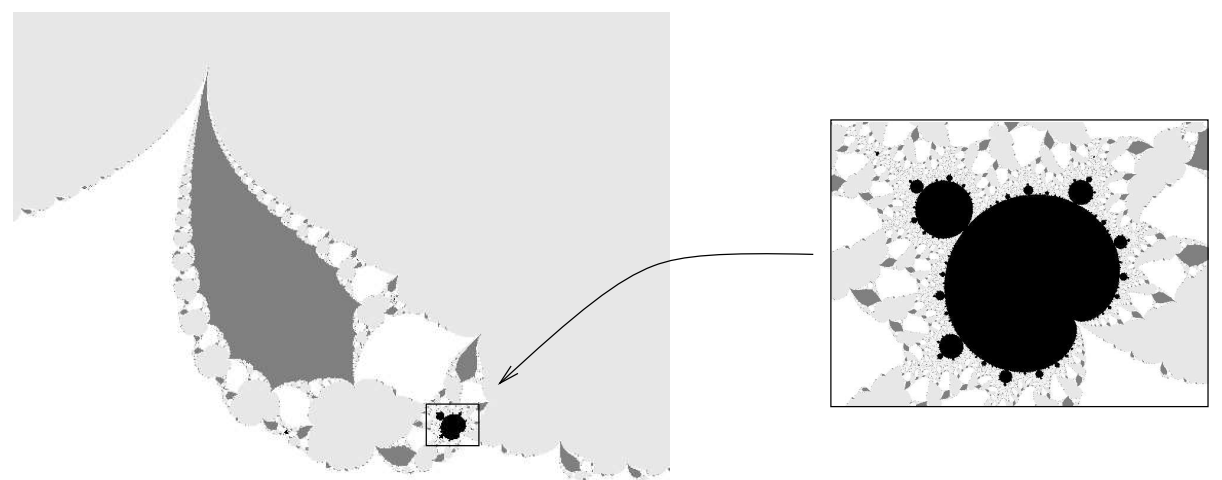

Fig. 3. A fragment of the parameter space of parabolic maps with supersinks $0, \infty$, and a fixed point of multiplier 1. Light grey colour corresponds to parameters for which the critical point 1 is in the parabolic basin. The enlargement shows the Mandelbrot-like set $M_{1}$.

Let us remark that compared to the general case, it is much easier to prove the existence of the above bifurcation for the "centre" of the main cardioid in the Mandelbrot-like sets, i.e. the bifurcation on the surface

$$
\operatorname{Per}(k)=\left\{(a, b) \in \widehat{\mathbb{C}} \times \widehat{\mathbb{C}} \backslash \text { Sing }: 1 \text { is periodic with period } k \text { for } f_{a, b}\right\} .
$$

(For $k=2$, this was done in Theorem I.4.20.) One can show that for some open set $\mathcal{V} \subset \mathbb{C} \times \mathbb{C}$ containing $\bigcup_{\varrho \in \mathbb{D} \cup\{1\}} \operatorname{Fix}(\varrho)$, the set $\operatorname{Per}(k) \cap \mathcal{V}$ is a one-dimensional complex manifold, transversal to the set of Newton maps with supersinks $0, u, \infty$. (The proof is similar to the proof of Theorem I.4.14.) Moreover, one can show there are no singular parameters in $\operatorname{cl} \operatorname{Per}(k) \cap \mathcal{V}$. Let $\xi_{a, b} \neq 0, \infty$ be the $f_{a, b}$-attracting fixed point for $(a, b) \in \operatorname{Per}(k) \cap \bigcup_{\varrho \in \mathbb{D}} \operatorname{Fix}(\varrho)$. Then the function $(a, b) \mapsto \xi_{a, b}$ is holomor- 


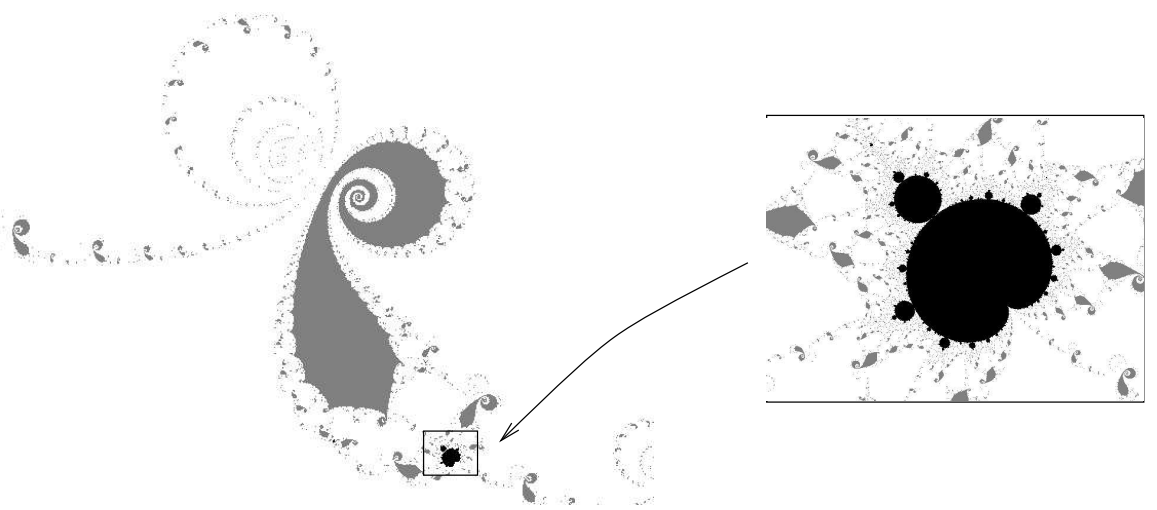

Fig. 4. A fragment of the parameter space of maps with supersinks $0, \infty$ such that $u$ is in the exotic basin of $\infty$. The enlargement shows the Mandelbrot-like set $M_{t}^{\infty}$.

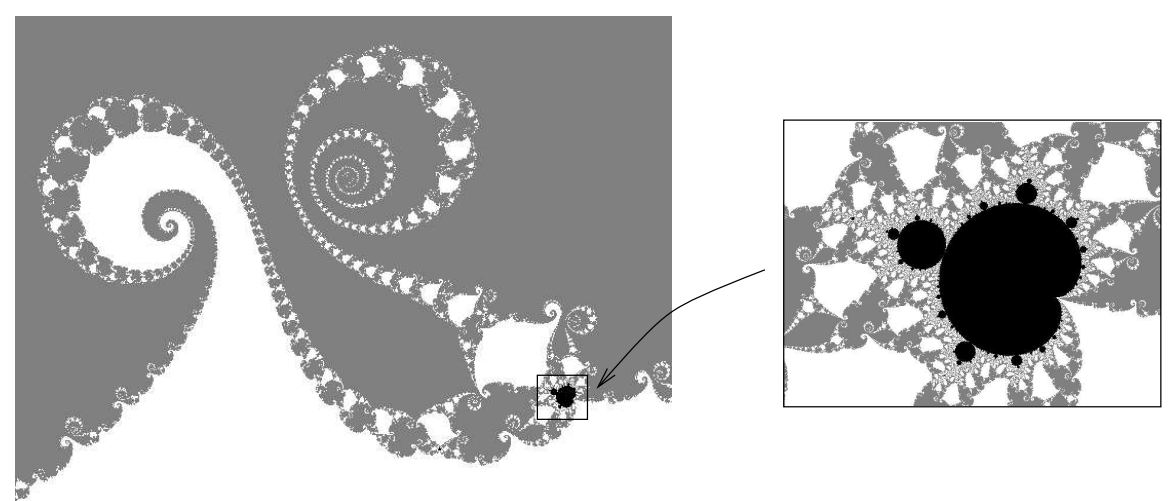

Fig. 5. A fragment of the parameter space of maps with supersinks $0, \infty$ such that $u$ is in the non-exotic basin of 0 . The enlargement shows the Mandelbrot-like set $M_{t}^{0}$.

phic and the boundary of $\operatorname{Per}(k) \cap \mathcal{V} \cap \bigcup_{\varrho \in \mathbb{D}} \operatorname{Fix}(\varrho)$ is a piecewise analytic curve in $\bigcup_{\varrho \in \partial \mathbb{D}} \operatorname{Fix}(\varrho)$. Hence, one can connect the map $N_{\sigma_{0}}$ to a parabolic map in $\operatorname{Per}(k) \cap \operatorname{Fix}(1)$ by a suitable curve $\gamma$ in $\operatorname{Per}(k) \cap \bigcup_{o \in \mathbb{D}} \operatorname{Fix}(\varrho)$. Then using the Douady-Sentenac theorem on the parabolic bifurcation (Theorem I.4.21) one can show the existence of two curves $\gamma_{0}, \gamma_{\infty}$ leading to maps for which the critical point $u$ is respectively in the (exotic or not) basin of 0 or $\infty$.

The proof of Theorem 4.1 in the general case is longer and consists of several parts. First, in Subsection 4.1 we show that we can modify the initial Mandelbrot-like family $\mathbf{N}$ to an equivalent family $\mathbf{f}_{0}=\left\{\left.N_{\sigma}^{k}\right|_{U_{\sigma}^{\prime}}\right\}_{\sigma \in \mathcal{U}^{\prime}}$ such that the topological discs $U_{\sigma}^{\prime}$ have a nice combinatorial description. In Subsection 4.2 we construct the family $\mathbf{f}_{t}$ for $t \in(0,1)$ using quasiconformal surgery. In Subsection 4.3 we define the family $\mathbf{f}_{1}$ and prove that $\left\{\mathbf{f}_{t}\right\}_{t \in[0,1]}$ is a continuous path of Mandelbrot-like families. This is the most technical 
part of the proof, which makes use of Lemmas 2.3 and 2.4. Subsection 4.4 contains the proof of the existence of the families $\mathbf{f}_{t}^{0}$ and $\mathbf{f}_{t}^{\infty}$, similar to the proof of Theorem I.4.20. In Subsection 4.5 we determine which path is exotic. Finally, in Subsection 4.6 we extend the non-exotic path so that it ends in the family of maps with a double critical point (conformally conjugate to cubic polynomials with a supersink), using quasiconformal surgery once more.

Consider the initial family $\mathbf{N}$ and the map $N_{\sigma_{0}}$. Then the boundaries of two of the three immediate basins $B(0), B(u), B(\infty)$ have an infinite intersection and the boundary of the third one has a one-point intersection with the others (see Subsection I.4.4). Hence, we have three possibilities: $\partial B(u) \cap \partial B(\infty)$ is infinite, $\partial B(u) \cap \partial B(0)$ is infinite or $\partial B(0) \cap \partial B(\infty)$ is infinite. We will consider only the first case (then, according to Theorem 4.1, the path $\left\{\mathbf{f}_{t}^{\infty}\right\}$ is exotic and $\left\{\mathbf{f}_{t}^{0}\right\}$ is non-exotic). The second case is symmetric to the first (it differs only by the exchange of 0 and $\infty$ ) and the third can be proved in the same way (in fact, it is technically much easier).

4.1. Modifying the sets $U_{\sigma}$. Recall that for $\sigma \in \mathcal{U}$ the set $U_{\sigma}$ is a topological disc and $N_{\sigma}^{k}$ is quadratic-like on $U_{\sigma}$. It is obvious that $U_{\sigma}$ contains the critical point 1 . Let $K_{\sigma}$ be the filled-in Julia set of $\left.N_{\sigma}^{k}\right|_{U_{\sigma}}$. We construct a topological disc $\mathcal{U}^{\prime} \subset \mathcal{U}$ containing $M_{N}$ and topological discs $U_{\sigma}^{\prime}$ for $\sigma \in \mathcal{U}^{\prime}$ such that $U_{\sigma}^{\prime}$ contains the critical point $1, K_{\sigma} \subset U_{\sigma}^{\prime}$ and $\left.N_{\sigma}^{k}\right|_{U_{\sigma}^{\prime}}$ is quadratic-like. The boundary of the sets $U_{\sigma}^{\prime}$ will consist of suitable parts of some equipotential curves and preperiodic internal rays in the basins of $0, u, \infty$.

Recall that for any Newton map $N_{\sigma}$ the three immediate basins $B_{\sigma}(\zeta)=$ $B(\zeta)$ for $\zeta \in\{0, u, \infty\}$ are simply connected and $N_{\sigma}$ has a unique repelling fixed point $p_{\sigma}=\partial B_{\sigma}(0) \cap \partial B_{\sigma}(u) \cap \partial B_{\sigma}(\infty)$ (see [P2]).

Definition 4.2. For $\zeta \in\{0, u, \infty\}$ and $\sigma \in \Sigma$ let $\psi_{\sigma}^{\zeta}$ be the unique Riemann mapping from $\mathbb{D}$ onto $B_{\sigma}(\zeta)$ such that $\psi_{\sigma}^{\zeta}(0)=\zeta$ and $\left(\psi_{\sigma}^{\zeta}\right)^{\prime}(0)>0$.

It is clear that $\psi_{\sigma}^{\zeta}$ conjugates $N_{\sigma}$ to

$$
\widetilde{h}_{\sigma}^{\zeta}(z)= \begin{cases}e^{2 \pi i \theta_{\sigma}^{\zeta}} z^{2} & \text { if } 1 \notin B_{\sigma}(\zeta), \\ e^{2 \pi i \theta_{\sigma}^{\zeta}} z^{2} \frac{z-\widetilde{\varrho}_{\sigma}^{\zeta}}{1-\widetilde{\varrho}_{\sigma}^{\zeta}} z & \text { if } 1 \in B_{\sigma}(\zeta),\end{cases}
$$

for some $\theta_{\sigma}^{\zeta} \in \mathbb{R} / \mathbb{Z}, \widetilde{\varrho}_{\sigma}^{\zeta} \in \mathbb{D}$.

Note that by [Ro], the boundaries of the immediate basins are locally connected. Hence, $\psi_{\sigma}^{\zeta}$ extends continuously to $\mathrm{cl} \mathbb{D}$. Moreover, by $[\mathrm{Ta}]$, if $1 \notin B_{\sigma}(\zeta)$, then $\partial B_{\sigma}(\zeta)$ is a Jordan curve, so by the Carathéodory theorem, the extension is a homeomorphism. If $1 \in B_{\sigma}(\zeta)$, then there are two $\widetilde{h}_{\sigma}^{\zeta}$-fixed points in $\partial \mathbb{D}$ and only one $N_{\sigma}$-fixed point $p_{\sigma} \in \partial B_{\sigma}(\zeta)$, so the extension is not a homeomorphism. 
It is easy to see that for $\zeta \in\{0, u, \infty\}$ and every sequence $\sigma_{n} \rightarrow \sigma$, $\sigma_{n}, \sigma \in \Sigma$ the maps $N_{\sigma_{n}}$ satisfy the assumptions of Lemma 2.3 with $\zeta_{n}=\zeta$. Hence, the maps $\psi_{\sigma}^{\zeta}$ and $\widetilde{h}_{\sigma}^{\zeta}$ depend continuously on $\sigma \in \Sigma$ in the almost uniform convergence topology, so $\theta_{\sigma}^{\zeta}, \widetilde{\varrho}_{\sigma}^{\zeta}$ are continuous functions of $\sigma \in \Sigma$.

Definition 4.3. For $\zeta \in\{0, u, \infty\}$ and $\sigma \in \Sigma$ let $\varphi_{\sigma}^{\zeta}$ be a Riemann mapping from $\mathbb{D}$ onto $B_{\sigma}(\zeta)$ such that $\varphi_{\sigma}^{\zeta}(0)=\zeta$ and $\varphi_{\sigma}^{\zeta}(1)=p_{\sigma}$.

The map $\varphi_{\sigma}^{\zeta}$ is a composition of $\psi_{\sigma}^{\zeta}$ with a suitable rotation in $\mathbb{D}$. If $1 \notin B_{\sigma}(\zeta)$, then $\varphi_{\sigma}^{\zeta}$ is uniquely determined and coincides with the inverse of the Böttcher coordinates (for the definition of the Böttcher coordinates see Subsection I.4.3). If $1 \in B_{\sigma}(\zeta)$, then there are two $\widetilde{h}_{\sigma}^{\zeta}$-fixed points in $\partial \mathbb{D}$, so there are two maps $\varphi_{\sigma}^{\zeta}$ satisfying Definition 4.3. Note that $\varphi_{\sigma}^{\zeta}$ conjugates $N_{\sigma}$ to

$$
h_{\sigma}^{\zeta}(z)= \begin{cases}z^{2} & \text { if } 1 \notin B_{\sigma}(\zeta), \\ \frac{1-\overline{\varrho_{\sigma}^{\zeta}}}{1-\varrho_{\sigma}^{\zeta}} z^{2} \frac{z-\varrho_{\sigma}^{\zeta}}{1-\varrho_{\sigma}^{\zeta} z} & \text { if } 1 \in B_{\sigma}(\zeta),\end{cases}
$$

for some $\varrho_{\sigma}^{\zeta} \in \mathbb{D}$.

Definition 4.4. For $\zeta \in\{0, u, \infty\}$ and $\sigma \in \Sigma$ such that $1 \notin B_{\sigma}(\zeta)$ let $R_{\zeta}(\theta)=\varphi_{\sigma}^{\zeta}\left(\left\{r e^{2 \pi i \theta}: r \in[0,1)\right\}\right)$ be the internal ray in $B_{\sigma}(\zeta)$ of angle $\theta \in$ $\mathbb{R} / \mathbb{Z}$ and let $l_{\zeta}(\theta)$ be its landing point. Set also $\widetilde{B}_{\sigma}(\zeta)=N_{\sigma}^{-1}\left(B_{\sigma}(\zeta)\right) \backslash B_{\sigma}(\zeta)$, $\widetilde{R}_{\zeta}(\theta)=N_{\sigma}^{-1}\left(R_{\zeta}(\theta)\right) \cap \widetilde{B}_{\sigma}(\zeta)$ and let $\widetilde{l}_{\zeta}(\theta)$ be the landing point of $\widetilde{R}_{\zeta}(\theta)$ (cf. Subsection I.4.4).

The case $\sigma \in M_{N}$. Consider first the map $N_{\sigma_{0}}$. Then $1 \notin B_{\sigma_{0}}(0) \cup$ $B_{\sigma_{0}}(u) \cup B_{\sigma_{0}}(\infty)$, so for $\zeta \in\{0, u, \infty\}$ the map $\varphi_{\sigma}^{\zeta}$ conjugates $N_{\sigma}$ on $B_{\sigma}(\zeta)$ to $z \mapsto z^{2}$. As was remarked, we assume that the set $\partial B_{\sigma_{0}}(u) \cap \partial B_{\sigma_{0}}(\infty)$ is infinite. The positions of the three basins are presented in Fig. I.6 and Fig. 7.

By assumption, Head's angle $\alpha$ (see Subsection I.4.4 for the definition) is periodic with period $k \bmod 1$, so $\alpha=m /\left(2^{k}-1\right)$ for some $m$. The angle $\beta$ defined in Subsection I.4.4 is equal to $m / 2^{k}$. Define also another angle $\delta=m / 2^{k}+1 / 2^{k+1}$. Since $\alpha<1 / 2$, we have $\beta<\alpha<\delta$. Using the definitions of $\alpha, \beta, \delta$ and the combinatorics of Newton maps described in Subsection I.4.4, it is easy to show that $l_{u}(2 \beta)=l_{\infty}(1-2 \beta), 2^{k-1} \beta=$ $1 / 2 \bmod 1, l_{u}(\delta)=l_{\infty}(1-\delta)$ and $2^{k} \delta=1 / 2 \bmod 1$. This implies that if we set $\tau(z)=2 z \bmod 1$, then

$$
\tau^{k}([\beta, \delta])=[0,1 / 2] \supset(0,1 / 2) \supset[\beta, \delta]
$$

and $\tau^{k}$ is injective on $[\beta, \delta]$. 
Definition 4.5. For $\sigma \in M_{N}$ let

$$
\begin{aligned}
& L_{\sigma}=\operatorname{cl}\left(R_{u}(\delta) \cup R_{\infty}(1-\delta) \cup R_{\infty}(1-\beta) \cup \widetilde{R}_{u}(2 \beta)\right. \\
&\left.\cup \widetilde{R}_{u}(2 \delta) \cup \widetilde{R}_{\infty}(1-2 \delta) \cup \widetilde{R}_{\infty}(1-2 \beta) \cup R_{u}(\beta)\right) .
\end{aligned}
$$

(See Fig. 6, where $L_{\sigma}$ is the dashed line.) The definitions of $\beta$ and $\delta$ easily imply that $L_{\sigma_{0}}$ is a Jordan curve.

Lemma 4.6. The set $L_{\sigma}$ is a Jordan curve for every $\sigma \in M_{N}$.

Proof. For $\sigma \in M_{N}$ the critical point 1 is contained in the filled-in Julia set $K_{\sigma}$, so it cannot be in the basin of a supersink. Hence, the rays in $B_{\sigma}(u), B_{\sigma}(\infty), \widetilde{B}_{\sigma}(u)$ and $\widetilde{B}_{\sigma}(\infty)$ are defined (and have well defined landing points). We need to prove that for $\sigma \in M_{N}$ we have $l_{u}(\delta)=l_{\infty}(1-\delta)$, $l_{\infty}(1-\beta)=\widetilde{l}_{u}(2 \beta), \widetilde{l}_{u}(2 \delta)=\widetilde{l}_{\infty}(1-2 \delta)$ and $\widetilde{l}_{\infty}(1-2 \beta)=l_{u}(\beta)$. First we show that for every $\sigma \in M_{N}$ the forward orbit of 1 does not hit the landing points of any of these rays. Since all these points are preimages of $p_{\sigma}=l_{0}(0)=l_{u}(0)=l_{\infty}(0)$ and $1 \in K_{\sigma}$, it is sufficient to prove that

$$
p_{\sigma} \notin K_{\sigma} \quad \text { for all } \sigma \in \mathcal{U} \text {. }
$$

To show (19), suppose that $p_{\sigma}$ is a fixed point of the quadratic-like map $\left.N_{\sigma}^{k}\right|_{U_{\sigma}}$ for some $\sigma \in \mathcal{U}$. Such a point moves continuously when we perturb $\sigma$ and for $\sigma=\chi^{-1}(1 / 4)$ becomes a parabolic point. This is not possible, since $p_{\sigma}$ is a repelling fixed point for all Newton maps. In this way we have shown (19), which implies that the forward orbit of 1 is disjoint from the landing points of the above rays.

Now we can prove the four equalities. We consider only the first oneothers can be shown in the same way. Let

$$
M^{\prime}=\left\{\sigma \in M_{N}: l_{u}(\delta)=l_{\infty}(1-\delta) \text { for } N_{\sigma}\right\} .
$$

We will show that $M^{\prime}$ is open and closed in $M_{N}$. As $\sigma_{0} \in M^{\prime}$ and $M_{N}$ is connected, this will imply $M^{\prime}=M_{N}$.

Take $\sigma \in M^{\prime}$. We have shown that the forward orbit of 1 is disjoint from $l_{u}(1 / 2)$. Hence, there exists a branch $\nu_{\sigma}$ of $N_{\sigma}^{-k}$ defined in some open neighbourhood of $R_{u}(1 / 2) \cup l_{u}(1 / 2) \cup R_{\infty}(1 / 2)$ such that

$$
\nu_{\sigma}\left(R_{u}(1 / 2) \cup l_{u}(1 / 2) \cup R_{\infty}(1 / 2)\right)=R_{u}(\delta) \cup l_{u}(\delta) \cup R_{\infty}(1-\delta) .
$$

As we perturb $\sigma$ within $M_{N}$, these rays are defined and move in a continuous way (this follows e.g. from Lemma 2.4). Therefore, for a small perturbation of $\sigma$ within $M_{N}$, the branch $\nu_{\sigma}$ exists and (20) holds. This shows that $M^{\prime}$ is open in $M_{N}$.

To see that $M^{\prime}$ is closed in $M_{N}$, suppose that we have a sequence $\sigma_{n} \in M^{\prime}$ such that $\sigma_{n} \rightarrow \sigma \in M_{N}$ and $\sigma \notin M^{\prime}$. Then $l_{u}(\delta)$ for $N_{\sigma}$ is in the boundary of some preimage of $B_{\sigma}(\infty)$ different from $B_{\sigma}(\infty)$. Hence, there exists $0 \leq j<k$ such that $l_{u}\left(2^{j} \delta\right) \in \widetilde{B}_{\sigma}(\infty)$, so $l_{u}\left(2^{j} \delta\right)=\widetilde{l}_{\infty}\left(1-2^{j+1} \delta\right)$. Therefore, there 
exists a branch $\widetilde{\nu}_{\sigma}$ of $N_{\sigma}^{-(k-j)}$ defined in some neighbourhood of $R_{u}(1 / 2) \cup$ $l_{u}(1 / 2) \cup R_{\infty}(1 / 2)$ such that

$$
\widetilde{\nu}_{\sigma}\left(R_{u}(1 / 2) \cup l_{u}(1 / 2) \cup R_{\infty}(1 / 2)\right)=R_{u}\left(2^{j} \delta\right) \cup l_{u}\left(2^{j} \delta\right) \cup \widetilde{R}_{\infty}\left(1-2^{j+1} \delta\right) .
$$

For a small perturbation of $\sigma$ in $M_{N}$ such a branch still exists. Thus, if we take sufficiently large $n$, then for the map $N_{\sigma_{n}}$ we have

$$
l_{u}\left(2^{j} \delta\right)=\widetilde{l}_{\infty}\left(1-2^{j+1} \delta\right) .
$$

But $\sigma_{n} \in M^{\prime}$, so for $N_{\sigma_{n}}$ we must have $l_{u}\left(2^{j} \delta\right)=l_{\infty}\left(1-2^{j} \delta\right)$. This is a contradiction. Therefore $\sigma \in M^{\prime}$, so $M^{\prime}$ is closed in $M_{N}$. We conclude that $M^{\prime}=M_{N}$.

By Lemma 4.6, we can state the following definition.

Definition 4.7. For $\sigma \in M_{N}$ let $E_{\sigma}$ be the component of $\widehat{\mathbb{C}} \backslash L_{\sigma}$ containing the critical point 1 and let

$$
U_{\sigma}^{\prime}=E_{\sigma} \backslash \operatorname{cl} N_{\sigma}^{-1}\left(\varphi_{\sigma}^{u}\left(\mathbb{D}_{\widehat{r}^{2}-k+1}\right) \cup \varphi_{\sigma}^{\infty}\left(\mathbb{D}_{\widehat{r}^{2}-k+1}\right)\right)
$$

for a fixed $\widehat{r}<1$ close to 1 .

By the definitions of $\beta, \delta$ and by (18), $U_{\sigma}^{\prime}$ is a topological disc containing 1 and $\left.N_{\sigma}^{k}\right|_{U_{\sigma}^{\prime}}$ is quadratic-like. Moreover,

$$
\begin{aligned}
& \partial N_{\sigma}\left(U_{\sigma}^{\prime}\right)=\varphi_{\sigma}^{u}(\left\{r e^{2 \pi i 2 \beta}: r \in\left[\widehat{r}^{2^{-k+1}}, 1\right]\right\} \\
& \cup\left\{\widehat{r}^{2^{-k+1}} e^{2 \pi i \theta}: \theta \in[2 \beta, 2 \delta]\right\} \\
& \cup\left.\left\{r e^{2 \pi i 2 \delta}: r \in\left[\widehat{r}^{2^{-k+1}}, 1\right]\right\}\right) \\
& \cup \varphi_{\sigma}^{\infty}\left(\left\{r e^{2 \pi i(1-2 \delta)}: r \in\left[\widehat{r}^{2^{-k+1}}, 1\right]\right\}\right. \\
& \cup\left\{\widehat{r}^{2^{-k+1}} e^{2 \pi i \theta}: \theta \in[1-2 \delta, 1-2 \beta]\right\} \\
&\left.\cup\left\{r e^{2 \pi i(1-2 \beta)}: r \in\left[\widehat{r}^{-k+1}, 1\right]\right\}\right), \\
& \partial N_{\sigma}^{k}\left(U_{\sigma}^{\prime}\right)=\varphi_{\sigma}^{u}\left([\widehat{r}, 1] \cup\left\{\widehat{r} e^{2 \pi i \theta}: \theta \in[0,1 / 2]\right\} \cup[-1,-\widehat{r}]\right) \\
& \cup \varphi_{\sigma}^{\infty}\left([\widehat{r}, 1] \cup\left\{\widehat{r} e^{2 \pi i \theta}: \theta \in[1 / 2,1]\right\} \cup[-1,-\widehat{r}]\right) .
\end{aligned}
$$

See Figs. 6 and 7.

Now we show

$$
K_{\sigma} \subset U_{\sigma}^{\prime} \quad \text { for all } \sigma \in M_{N} \text {. }
$$

The boundary of $U_{\sigma}^{\prime}$ consists of points from the basins of attraction to $u$ and $\infty$ and the landing points of some rays. It is obvious that $K_{\sigma}$ cannot contain points from the basins of $u$ and $\infty$. Since $1 \in K_{\sigma}$ and $K_{\sigma}$ is connected for $\sigma \in M_{N}$, either $K_{\sigma} \subset U_{\sigma}^{\prime}$ or $K_{\sigma}$ contains the landing point of some ray from $\partial U_{\sigma}^{\prime}$. But the second case is impossible by (19). Hence, (21) holds.

We have constructed a family $\left\{U_{\sigma}^{\prime}\right\}_{\sigma \in M_{N}}$ of topological discs such that $\left.N_{\sigma}^{k}\right|_{U_{\sigma}^{\prime}}$ is quadratic-like and $K_{\sigma} \subset U_{\sigma}^{\prime}$. This implies (see e.g. [McM]) that for $\sigma \in M_{N}$ the filled-in Julia sets $K_{\sigma}$ of $\left.N_{\sigma}^{k}\right|_{U_{\sigma}}$ and $K_{\sigma}^{\prime}$ of $\left.N_{\sigma}^{k}\right|_{U_{\sigma}^{\prime}}$ coincide. 


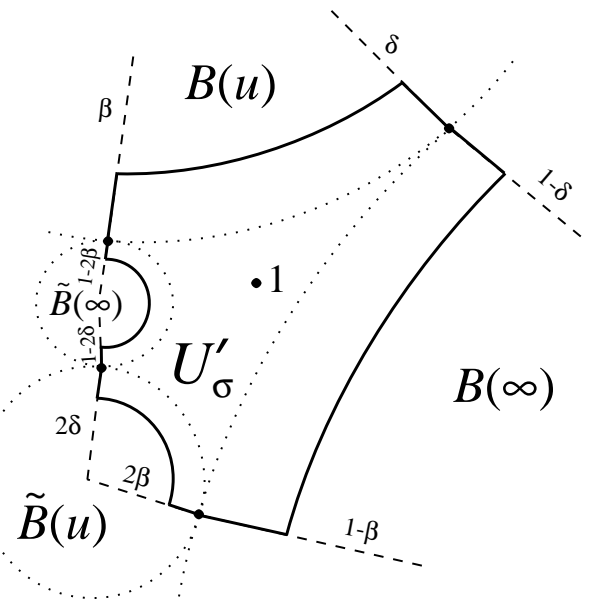

Fig. 6. The set $U_{\sigma}^{\prime}$

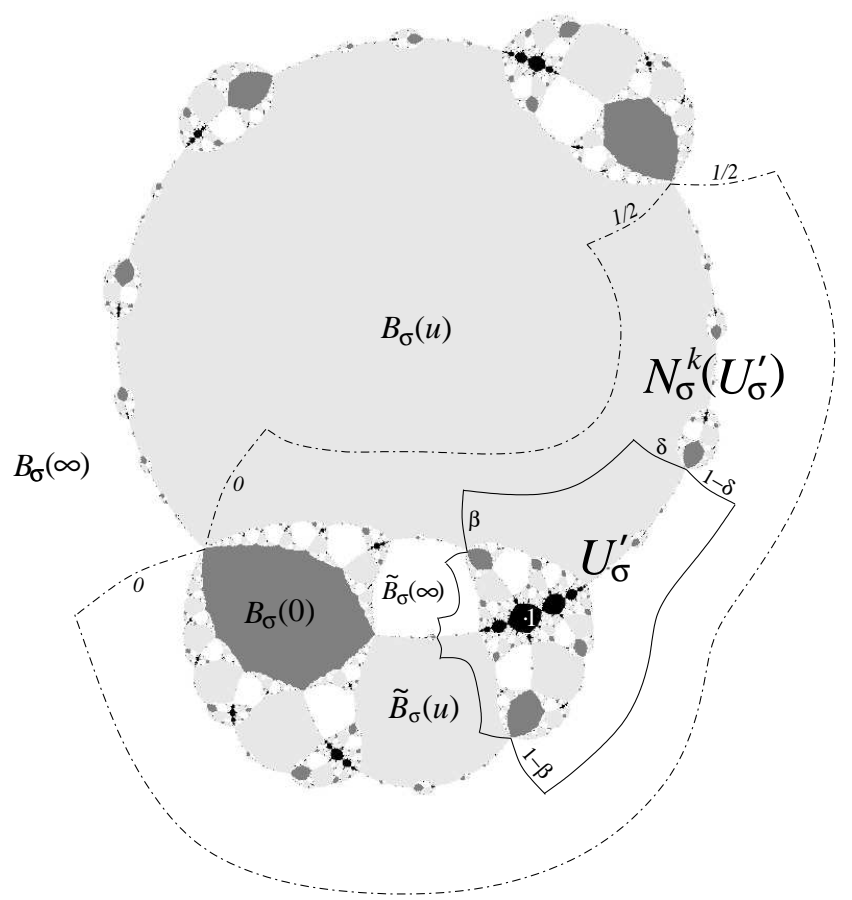

Fig. 7. The set $U_{\sigma}^{\prime}$ in the dynamical plane

The case $\sigma \notin M_{N}$. Now we extend the definition of $U_{\sigma}^{\prime}$ for $\sigma$ in some topological disc $\mathcal{U}^{\prime}$ such that $M_{N} \subset \mathcal{U}^{\prime} \subset \mathcal{U}$. First we show that for $\zeta \in\{u, \infty\}$ and $\sigma$ in some neighbourhood of $M_{N}$, if $1 \in B_{\sigma}(\zeta)$, then we 
can choose one of two Riemann maps $\varphi_{\sigma}^{\zeta}$ from Definition 4.3 such that $\varphi_{\sigma}^{\zeta}$ depends continuously on $\sigma$. To do it, we prove the following technical lemma:

LEMmA 4.8. There exist $\varepsilon_{0}>0$ and an open set $\widetilde{\mathcal{U}} \subset \Sigma$ containing $M_{N}$ such that for every $\sigma \in \widetilde{\mathcal{U}}$ and $\zeta \in\{u, \infty\}$ there exists an $\widetilde{h}_{\sigma}^{\zeta}$-fixed point $q_{\sigma}^{\zeta} \in \partial \mathbb{D}$ such that

$$
\text { if } 1 \in B_{\sigma}(\zeta) \text {, then }\left|\widetilde{v}_{\sigma}^{\zeta}-q_{\sigma}^{\zeta}\right|>\varepsilon_{0},
$$

where $\widetilde{h}_{\sigma}^{\zeta}$ is the map from (16) and $\widetilde{v}_{\sigma}^{\zeta}=\left(\psi_{\sigma}^{\zeta}\right)^{-1}(1)$ is the unique non-zero $\tilde{h}_{\sigma}^{\zeta}$-critical point in $\mathbb{D}$.

Proof. To simplify notation, consider only the case $\zeta=u$. Suppose the lemma is not true. Then there exists a sequence $\sigma_{n} \in \Sigma$ such that $\sigma_{n} \rightarrow$ $\sigma \in M_{N}$, for every $n$ we have $1 \in B_{\sigma_{n}}(u)$ and

$$
\left|\widetilde{v}_{\sigma_{n}}^{u}-q_{\sigma_{n}}\right|,\left|\widetilde{v}_{\sigma_{n}}^{u}-\widetilde{q}_{\sigma_{n}}\right| \rightarrow 0 \quad \text { as } n \rightarrow \infty,
$$

where $q_{\sigma_{n}}, \widetilde{q}_{\sigma_{n}}$ are $\widetilde{h}_{\sigma_{n}}^{u}$-fixed points in $\partial \mathbb{D}$. For simplicity, set $h_{n}=\widetilde{h}_{\sigma_{n}}^{u}$, $v_{n}=\widetilde{v}_{\sigma_{n}}^{u}, q_{n}=q_{\sigma_{n}}$ and $\widetilde{q}_{n}=\widetilde{q}_{\sigma_{n}}$. By Lemma $2.3, h_{n}$ tends to $\widetilde{h}_{\sigma}(z)=$ $e^{2 \pi i \theta_{\sigma}} z^{2}$ and $\psi_{\sigma_{n}}^{u}$ tends to $\psi_{\sigma}^{u}$. Changing $\psi_{\sigma_{n}}^{u}, \psi_{\sigma}^{u}$ by the rotation by $\theta_{\sigma}$, assume $\psi_{\sigma}^{u}=\varphi_{\sigma}^{u}$ so that $h_{n}(z) \rightarrow h(z)=z^{2}$. By Lemma $2.3, h_{n} \rightarrow h$ almost uniformly in the spherical metric on $\widehat{\mathbb{C}} \backslash\left\{z_{0}\right\}$ for some $z_{0} \in \partial D$. As 1 is the only $h$-fixed point in $\partial \mathbb{D}$, this easily implies that $q_{n}, \widetilde{q}_{n} \rightarrow 1$ and $z_{0}=1$. Thus, we have

$$
v_{n} \rightarrow 1
$$

Let $V$ be a small neighbourhood of the repelling $N_{\sigma}$-fixed point $p=p_{\sigma}$. Then there exists a branch $\nu$ of $N_{\sigma}^{-1}$ defined on $V$ such that $\nu(p)=p$ and $\operatorname{cl} \nu(V) \subset V$. Moreover, since $N_{\sigma_{n}} \rightarrow N_{\sigma}$ uniformly in the spherical metric on $\widehat{\mathbb{C}}$, for sufficiently large $n$ there exists a branch $\nu_{n}$ of $N_{\sigma_{n}}^{-1}$ defined on $V$ such that $\nu_{n}\left(p_{\sigma_{n}}\right)=p_{\sigma_{n}}$ and $\operatorname{cl} \nu_{n}(V) \subset V$.

Let $\Phi_{n}$ be the Böttcher coordinates for $h_{n}$ on $\mathbb{D}$. Note that for sufficiently large $n$ the map $\Phi_{n}^{-1}$ is defined on $\operatorname{cl} \mathbb{D}_{\widehat{r}^{2}-k}$ for $\widehat{r}$ from Definition 4.7 and $\Phi_{n} \rightarrow$ id uniformly on this set. Let

$$
I_{n}=\Phi_{n}^{-1}\left(\left[\widehat{r}^{2}, \widehat{r}\right]\right) .
$$

By Lemma 2.3, we can assume $\psi_{\sigma_{n}}^{u}\left(I_{n}\right) \subset V$ for large $n$ provided $\widehat{r}$ is fixed sufficiently close to 1 . Define a curve $\gamma_{n}:[0, \infty) \rightarrow \mathbb{D}$ setting $\gamma_{n}(s)=$ $\Phi_{n}^{-1}\left((1-s) \widehat{r}^{2}+s \widehat{r}\right)$ for $s \in[0,1]$ and $\gamma_{n}(s+1)=\left(\psi_{\sigma_{n}}^{u}\right)^{-1}\left(\nu_{n}\left(\psi_{\sigma_{n}}^{u}\left(\gamma_{n}(s)\right)\right)\right)$ for $s>1$. Define also a curve $\gamma:[0, \infty) \rightarrow \mathbb{D}$ parameterizing the segment $\left[\widehat{r}^{2}, 1\right)$ such that $\gamma(0)=\widehat{r}^{2}, \gamma(1)=\widehat{r}, \gamma$ is affine on $[0,1]$ and $\gamma(s+1)=\sqrt{\gamma(s)}$. Since $1 \notin V$, we have $v_{n}=\left(\psi_{\sigma_{n}}^{u}\right)^{-1}(1) \notin \gamma_{n}$, so there exist two branches $\nu_{n}^{\prime}, \nu_{n}^{\prime \prime}$ of $h_{n}^{-1}$ defined in a neighbourhood of $\gamma_{n}$ such that $\nu_{n}^{\prime}\left(\gamma_{n}(0)\right)=\Phi_{n}^{-1}(\widehat{r})$ 
and $\nu_{n}^{\prime \prime}\left(\gamma_{n}(0)\right)=\Phi_{n}^{-1}(-\widehat{r})$. Let

$$
\gamma_{n}^{\prime}=\nu_{n}^{\prime}\left(\gamma_{n}\right), \quad \gamma_{n}^{\prime \prime}=\nu_{n}^{\prime \prime}\left(\gamma_{n}\right)
$$

and define

$$
\begin{aligned}
\Gamma_{n}^{u} & =\gamma_{n}^{\prime} \cup \Phi_{n}^{-1}\left(\partial \mathbb{D}_{\widehat{r}} \cap \mathbb{D}^{+}\right) \cup \gamma_{n}^{\prime \prime}, \\
\Gamma^{u} & =[\widehat{r}, 1) \cup\left(\partial \mathbb{D}_{\widehat{r}} \cap \mathbb{D}^{+}\right) \cup(-1,-\widehat{r}], \\
\Gamma^{\infty} & =[\widehat{r}, 1) \cup\left(\partial \mathbb{D}_{\widehat{r}} \cap \mathbb{D}^{-}\right) \cup(-1,-\widehat{r}] .
\end{aligned}
$$

Note that since $1 \notin B_{\sigma_{n}}(\infty), B_{\sigma}(\infty)$, the maps $\varphi_{\sigma_{n}}^{\infty}, \varphi_{\sigma}^{\infty}$ are uniquely determined and coincide with the inverse of the Böttcher coordinates. Now we show that for sufficiently large $n$,

$\operatorname{cl}\left(\psi_{\sigma_{n}}^{u}\left(\Gamma_{n}^{u}\right) \cup \varphi_{\sigma_{n}}^{\infty}\left(\Gamma^{\infty}\right)\right)$ is a Jordan curve converging to $\partial N_{\sigma}^{k}\left(U_{\sigma}^{\prime}\right)$ in the Hausdorff metric.

Note that the curves $\psi_{\sigma_{n}}^{u}\left(\gamma_{n}^{\prime}\right)$ and $\varphi_{\sigma_{n}}^{\infty}([\widehat{r}, 1))$ land at the same point $p_{\sigma_{n}}$ and $\varphi_{\sigma_{n}}^{\infty}((-1,-\widehat{r}])$ lands at $l_{\infty}(1 / 2)$ for the map $N_{\sigma_{n}}$. Hence, to prove that $\operatorname{cl}\left(\psi_{\sigma_{n}}^{u}\left(\Gamma_{n}^{u}\right) \cup \psi_{\sigma_{n}}^{\infty}\left(\Gamma^{\infty}\right)\right)$ is a Jordan curve, we only need to show that for sufficiently large $n$ the curve $\psi_{\sigma_{n}}^{u}\left(\gamma_{n}^{\prime \prime}\right)$ lands at $l_{\infty}(1 / 2)$ for the map $N_{\sigma_{n}}$.

By Lemma 2.3, $\gamma_{n}(s)$ tends to $\gamma(s)$ almost uniformly on $[0, \infty)$ and $\gamma_{n}, \gamma$ satisfy the assumptions of the case (a) of Lemma 2.4. By this lemma, for arbitrarily small $\varepsilon>0$ there exists $s_{0}>0$ such that $\psi_{\sigma_{n}}^{u}\left(\gamma_{n}\left(\left[s_{0}, \infty\right)\right)\right) \subset \mathbb{D}_{\varepsilon}(p)$ for large $n$. By (19), $N_{\sigma}$ maps biholomorphically a small neighbourhood of $x=l_{u}(1 / 2)=l_{\infty}(1 / 2)$ onto a small neighbourhood of $p$. Moreover, there exists a small $\varepsilon^{\prime}>0$ such that $N_{\sigma_{n}}$ for large $n$ maps biholomorphically $\mathbb{D}_{\varepsilon^{\prime}}(x)$ onto a set containing $\mathbb{D}_{\varepsilon}(p)$. Since $x$ is the landing point of $\psi_{\sigma}^{u}((-1,-\widehat{r}])$ and $\varphi_{\sigma}^{\infty}((-1,-\widehat{r}])$, by almost uniform convergence, $\mathbb{D}_{\varepsilon^{\prime}}(x)$ contains points from $\psi_{\sigma_{n}}^{u}\left(\gamma_{n}^{\prime \prime}\right)$ and $\varphi_{\sigma_{n}}^{\infty}((-1,-\widehat{r}])$ for large $n$. Therefore, $\psi_{\sigma_{n}}^{u}\left(\gamma_{n}^{\prime \prime}\right)$ and $\varphi_{\sigma_{n}}^{\infty}((-1,-\widehat{r}])$ must land at the same point near $x$. In this way we have proved that $\operatorname{cl}\left(\psi_{\sigma_{n}}^{u}\left(\Gamma_{n}\right) \cup \varphi_{\sigma_{n}}^{\infty}(\Gamma)\right)$ is a Jordan curve for large $n$. Moreover, Lemma 2.4 implies that $\mathrm{cl} \gamma_{n}$ tends to $\mathrm{cl} \gamma$ and $\operatorname{cl} \psi_{\sigma_{n}}^{u}\left(\gamma_{n}\right)$ tends to $\operatorname{cl} \psi_{\sigma}^{u}(\gamma)$ in the Hausdorff metric. Since $\Phi_{n} \rightarrow$ id uniformly on $\mathrm{cl} \mathbb{D}_{\widehat{r}}, h_{n} \rightarrow h$ uniformly on $\mathrm{cl} \mathbb{D} \backslash\{1\}$ and $\psi_{\sigma_{n}}^{u} \rightarrow \psi_{\sigma}^{u}$ almost uniformly on $\mathbb{D}$, we conclude that $\mathrm{cl} \Gamma_{n}^{u}$ tends to $\mathrm{cl} \Gamma^{u}$ in the Hausdorff metric.

It is easy to check that the case (a) of Lemma 2.4 holds for $\gamma_{n}^{\infty}=\gamma^{\infty}=$ $[\widehat{r}, 1)$ with a suitable parameterization and $\varphi_{\sigma_{n}}^{\infty}, \varphi_{\sigma}^{\infty}$ instead of $\psi_{n}, \psi$. Repeating the above arguments we show that $\operatorname{cl} \varphi_{\sigma_{n}}^{\infty}\left(\Gamma^{\infty}\right)$ tends to $\operatorname{cl} \varphi_{\sigma}^{\infty}\left(\Gamma^{\infty}\right)$ in the Hausdorff metric. Hence, $\operatorname{cl}\left(\psi_{\sigma_{n}}^{u}\left(\Gamma_{n}^{u}\right) \cup \varphi_{\sigma_{n}}^{\infty}\left(\Gamma^{\infty}\right)\right)$ tends to $\operatorname{cl}\left(\psi_{\sigma}^{u}\left(\Gamma^{u}\right) \cup\right.$ $\left.\varphi_{\sigma}^{\infty}\left(\Gamma^{\infty}\right)\right)=\partial N_{\sigma}^{k}\left(U_{\sigma}^{\prime}\right)$ in the Hausdorff metric, which gives (23).

By (23), we can define $A_{n}$ for large $n$ to be the component of $\widehat{\mathbb{C}}$ । $\operatorname{cl}\left(\psi_{\sigma_{n}}^{u}\left(\Gamma_{n}^{u}\right) \cup \varphi_{\sigma_{n}}^{\infty}\left(\Gamma^{\infty}\right)\right)$ containing $N_{\sigma_{n}}^{k}(1)$ and $U_{n}$ to be the component of $N_{\sigma_{n}}^{-k}\left(A_{n}\right)$ containing 1 . Then $U_{n}$ is a topological disc and $\operatorname{cl} U_{n}$ tends to $\operatorname{cl} U_{\sigma}^{\prime}$ in the Hausdorff metric. Moreover, $\Gamma_{n}^{u}=\left(\psi_{\sigma_{n}}^{u}\right)^{-1}\left(\partial N_{\sigma_{n}}^{k}\left(U_{n}\right) \cap B_{\sigma_{n}}(u)\right)$. 
It is obvious that $h_{n}^{j}(0) \notin \Gamma_{n}^{u}$ for $j=0, \ldots, k$. Moreover, for large $n$ we have $N_{\sigma_{n}}^{j}(1) \in U_{n}$ for $j=0, \ldots, k$, so $h_{n}^{j}\left(v_{n}\right) \notin \Gamma_{n}^{u}$. Hence, there exists a branch $\widetilde{\nu}_{n}$ of $h_{n}^{-k}$ defined in a neighbourhood of $\Gamma_{n}^{u}$ such that

$$
\widetilde{\nu}_{n}\left(\Phi_{n}^{-1}\left(\partial \mathbb{D}_{\widehat{r}} \cap \mathbb{D}^{+}\right)\right)=\Phi_{n}^{-1}\left(\left\{\widehat{r}^{2^{-k}} e^{2 \pi i \theta}: \theta \in(\beta, \delta)\right\}\right) .
$$

Then $\widetilde{\nu}_{n}\left(\Gamma_{n}^{u}\right)$ is a simple arc in $\mathbb{D}$ with endpoints landing at two points in $\partial \mathbb{D}$. Let $Y_{n}$ be the component of $\mathbb{D} \backslash \widetilde{\nu}_{n}\left(\Gamma_{n}^{u}\right)$ which does not contain 0 . Then

$$
Y_{n} \supset\left(\psi_{\sigma_{n}}^{u}\right)^{-1}\left(U_{n} \cap B_{\sigma_{n}}(u)\right) .
$$

Let

$$
Y=\left\{r e^{2 \pi i \theta}: r \in\left[\widehat{r}^{2^{-k}}, 1\right), \theta \in(\beta, \delta)\right\} .
$$

By Definition 4.7,

$$
Y=\left(\psi_{\sigma}^{u}\right)^{-1}\left(U_{\sigma}^{\prime} \cap B_{\sigma}(u)\right), \quad h^{k}(\partial Y \cap \mathbb{D})=\Gamma^{u} .
$$

Recall that $\mathrm{cl} \Gamma_{n}^{u}$ tends to $\mathrm{cl} \Gamma^{u}$ in the Hausdorff metric, $\Phi_{n} \rightarrow$ id uniformly on $\mathrm{cl} \mathbb{D}_{\widehat{r}^{2}-k}$ and $h_{n} \rightarrow h$ uniformly on $\mathrm{cl} \mathbb{D} \backslash\{1\}$. This easily implies that $\partial Y_{n}$ tends to $\partial Y$ in the Hausdorff metric. Hence, $\operatorname{dist}\left(\operatorname{cl} Y_{n}, 1\right)>c$ for a constant $c>0$ independent of $n$. But since $1 \in U_{n} \cap B_{\sigma_{n}}(u)$, we have $v_{n}=\left(\psi_{\sigma_{n}}^{u}\right)^{-1}(1) \in Y_{n}$, so $\left|v_{n}-1\right|>c$, which contradicts $(22)$.

REMARK. In fact, one can show in the same way that if $\zeta \in\{u, \infty\}$ and $\sigma_{n} \rightarrow \sigma \in M_{N}$ with $1 \in B_{\sigma_{n}}(\zeta)$, then $\widetilde{v}_{\sigma_{n}}^{\zeta} \rightarrow e^{2 \pi i\left(\alpha-\theta_{\sigma}^{\zeta}\right)}$ for Head's angle $\alpha$ and $\theta_{\sigma}^{\zeta}$ from (16).

Lemma 4.8 implies that for every $\sigma \in \widetilde{\mathcal{U}}$ and $\zeta \in\{u, \infty\}$ there exists an $\widetilde{h}_{\sigma}^{\zeta}$-fixed point $q_{\sigma}^{\zeta} \in \partial \mathbb{D}$ depending continuously on $\sigma$, such that if $1 \in B_{\sigma}(\zeta)$, then for the other $\widetilde{h}_{\sigma}^{\zeta}$-fixed point $\widetilde{q}_{\sigma}^{\zeta} \in \partial \mathbb{D}$ we have $\left|q_{\sigma}^{\zeta}-\widetilde{q}_{\sigma}^{\zeta}\right|>\varepsilon_{0}$. This easily gives

COROLlary 4.9. There exists a topological disc $\mathcal{U}^{\prime} \subset \mathcal{U}$ containing $M_{N}$ such that for each $\sigma \in \mathcal{U}^{\prime}$ and $\zeta \in\{u, \infty\}$, if $1 \in B_{\sigma}(\zeta)$, then we can choose a Riemann map $\varphi_{\sigma}^{\zeta}$ from Definition 4.3 such that $\varphi_{\sigma}^{\zeta}$ depends continuously on $\sigma \in \mathcal{U}^{\prime}$ in the almost uniform convergence topology. In particular, Lemmas 2.3 and 2.4 hold for $N_{\sigma}, \sigma \in \mathcal{U}^{\prime}$ and $\varphi_{\sigma}^{\zeta}$ instead of $\psi_{\sigma}^{\zeta}$. Moreover, there exists $\varepsilon_{0}>0$ such that if $1 \in B_{\sigma}(\zeta)$, then

$$
\left|v_{\sigma}^{\zeta}-1\right|>\varepsilon_{0},
$$

where $v_{\sigma}^{\zeta}$ is the unique non-zero $h_{\sigma}^{\zeta}$-critical point in $\mathbb{D}$ for $h_{\sigma}^{\zeta}$ from (17).

From now on, by $\varphi_{\sigma}^{\zeta}$ we will always understand the map from the above corollary.

Let $\mathcal{U}^{\prime}$ be the topological disc from Corollary 4.9. Now we define $U_{\sigma}^{\prime}$ for $\sigma \in \mathcal{U}^{\prime}$. For $\zeta \in\{u, \infty\}$ let $\Phi_{\sigma}^{\zeta}$ be the Böttcher coordinates for $h_{\sigma}^{\zeta}$ on $\mathbb{D}$. If 
we take $\mathcal{U}^{\prime}$ sufficiently close to $M_{N}$, then $\left(\Phi_{\sigma}^{\zeta}\right)^{-1}$ is defined on $\operatorname{cl} \mathbb{D}_{\widehat{r}}$ for $\widehat{r}$ from Definition 4.7. Let

$$
I_{\sigma}^{\zeta}=\left(\Phi_{\sigma}^{\zeta}\right)^{-1}\left(\left[\widehat{r}^{2}, \widehat{r}\right]\right) .
$$

By Lemma 2.3 and Corollary 4.9, if we take $\mathcal{U}^{\prime}$ sufficiently close to $M_{N}$ and choose $\widehat{r}$ sufficiently close to 1 , then for every $\sigma \in \mathcal{U}^{\prime}$ there exist two branches $\nu_{\sigma}^{\prime \zeta}, \nu_{\sigma}^{\prime \prime \zeta}$ of $\left(h_{\sigma}^{\zeta}\right)^{-1}$ defined in a neighbourhood $V$ of 1 such that $I_{\sigma}^{\zeta} \subset V, \operatorname{cl} \nu_{\sigma}^{\prime \zeta}(V) \subset V, \nu_{\sigma}^{\prime \zeta}\left(\left(\Phi_{\sigma}^{\zeta}\right)^{-1}\left(\widehat{r}^{2}\right)\right)=\left(\Phi_{\sigma}^{\zeta}\right)^{-1}(\widehat{r}), \nu_{\sigma}^{\prime \prime \zeta}\left(\left(\Phi_{\sigma}^{\zeta}\right)^{-1}\left(\widehat{r}^{2}\right)\right)=$ $\left(\Phi_{\sigma}^{\zeta}\right)^{-1}(-\widehat{r})$. Connect $I_{\sigma}^{\zeta}$ to 1 by the curve $\gamma_{\sigma}^{\zeta}=\bigcup_{n=0}^{\infty}\left(\nu_{\sigma}^{\zeta}\right)^{n}\left(I_{\sigma}^{\zeta}\right)$ and let

$$
\gamma_{\sigma}^{\prime \zeta}=\nu_{\sigma}^{\prime \zeta}\left(\gamma_{\sigma}^{\zeta}\right), \quad \gamma_{\sigma}^{\prime \prime \zeta}=\nu_{\sigma}^{\prime \prime \zeta}\left(\gamma_{\sigma}^{\zeta}\right) \text {. }
$$

Then $\gamma_{\sigma}^{\prime \zeta}$ begins at $\left(\Phi_{\sigma}^{\zeta}\right)^{-1}(\widehat{r})$ and $\gamma_{\sigma}^{\prime \prime \zeta}$ begins at $\left(\Phi_{\sigma}^{\zeta}\right)^{-1}(-\widehat{r})$. Define

$$
\Gamma_{\sigma}^{\zeta}=\gamma_{\sigma}^{\prime \zeta} \cup\left(\Phi_{\sigma}^{\zeta}\right)^{-1}\left(\partial \mathbb{D}_{\widehat{r}} \cap \mathbb{D}^{ \pm}\right) \cup \gamma_{\sigma}^{\prime \prime \zeta}
$$

where we take $\mathbb{D}^{+}$for $\zeta=u$ and $\mathbb{D}^{-}$for $\zeta=\infty$. Then $\Gamma_{\sigma}^{\zeta}$ is a simple arc in $\mathbb{D}$ connecting 1 to some point of $\left(h_{\sigma}^{\zeta}\right)^{-1}(1)$ (see Fig. 8).

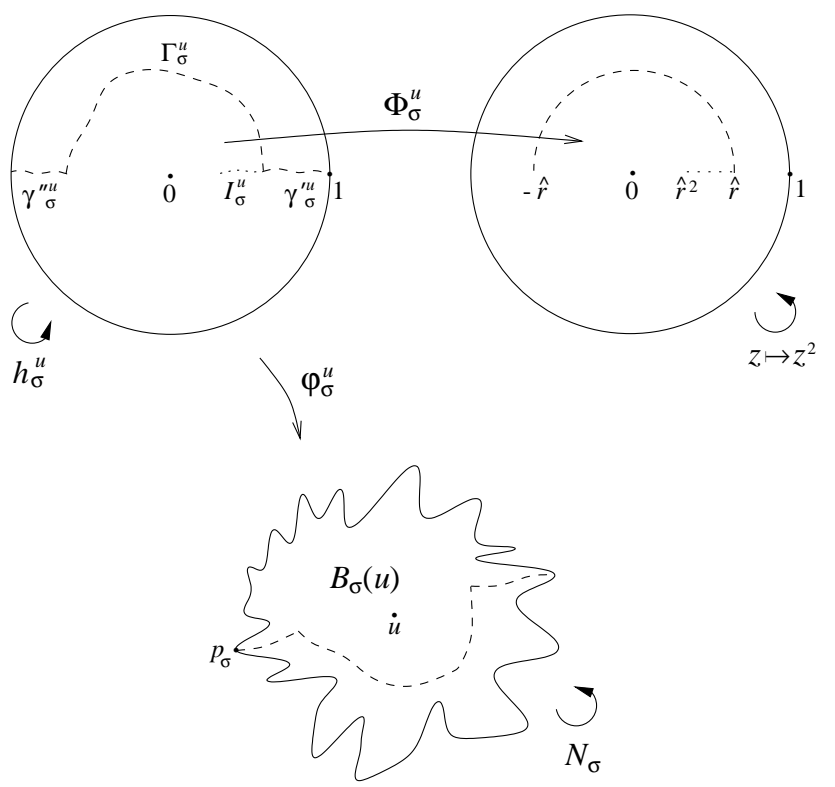

Fig. 8. The curves $\gamma_{\sigma}^{\prime u}, \gamma_{\sigma}^{\prime \prime}$ and $\Gamma_{\sigma}^{u}$

Now we show that if we take $\mathcal{U}^{\prime}$ sufficiently close to $M_{N}$, then

(25) for every $\sigma \in \mathcal{U}^{\prime}, \operatorname{cl}\left(\varphi_{\sigma}^{u}\left(\Gamma_{\sigma}^{u}\right) \cup \varphi_{\sigma}^{\infty}\left(\Gamma_{\sigma}^{\infty}\right)\right)$ is a Jordan curve depending continuously on $\sigma$ in the Hausdorff metric.

The proof of (25) is the same as for (23) and we leave it to the reader (by Corollary 4.9, we can use Lemmas 2.3 and 2.4 for $\varphi_{\sigma}^{\zeta}$ instead of $\psi_{\sigma}^{\zeta}$ ). Note 
that for $\sigma \in M_{N}$ we have $\varphi_{\sigma}^{\zeta}\left(\Gamma_{\sigma}^{\zeta}\right)=\partial N_{\sigma}^{k}\left(U_{\sigma}^{\prime}\right) \cap B_{\sigma}(\zeta)$ and $\operatorname{cl}\left(\varphi_{\sigma}^{u}\left(\Gamma_{\sigma}^{u}\right) \cup\right.$ $\left.\varphi_{\sigma}^{\infty}\left(\Gamma_{\sigma}^{\infty}\right)\right)=\partial N_{\sigma}^{k}\left(U_{\sigma}^{\prime}\right)$. By $(25)$, we can make the following definition.

Definition 4.10. For $\sigma \in \mathcal{U}^{\prime}$ let $A_{\sigma}$ be the component of $\widehat{\mathbb{C}} \backslash \operatorname{cl}\left(\varphi_{\sigma}^{u}\left(\Gamma_{\sigma}^{u}\right) \cup\right.$ $\left.\varphi_{\sigma}^{\infty}\left(\Gamma_{\sigma}^{\infty}\right)\right)$ containing $N_{\sigma}^{k}(1)$ and let $U_{\sigma}^{\prime}$ be the component of $N_{\sigma}^{-k}\left(A_{\sigma}\right)$ containing 1 .

Then (provided $\mathcal{U}^{\prime}$ is sufficiently close to $M_{N}$ ) the set $U_{\sigma}^{\prime}$ is a topological disc and $N_{\sigma}^{k}$ is quadratic-like on $U_{\sigma}^{\prime}$. Moreover, $\mathrm{cl} U_{\sigma}^{\prime}$ is continuous with respect to $\sigma \in \mathcal{U}^{\prime}$ in the Hausdorff metric and for $\sigma \in M_{N}$ the definition of $U_{\sigma}^{\prime}$ coincides with the previous one.

For $\sigma \in \mathcal{U}^{\prime}$ let $K_{\sigma}^{\prime}$ be the filled-in Julia set of $\left.N_{\sigma}^{k}\right|_{U_{\sigma}^{\prime}}$. Since diam $K_{\sigma}$ and diam $K_{\sigma}^{\prime}$ are continuous functions of $\sigma$ (see $[\mathrm{McM}]$ ), we can assume that $K_{\sigma} \subset U_{\sigma}^{\prime}$ for all $\sigma \in \mathcal{U}^{\prime}$. As previously, this gives $K_{\sigma}^{\prime}=K_{\sigma}$.

The above properties imply that $\left\{\left.N_{\sigma}^{k}\right|_{U_{\sigma}^{\prime}}\right\}_{\sigma \in \mathcal{U}^{\prime}}$ is a Mandelbrot-like family equivalent to $\mathbf{N}$ (see Section 3 ).

4.2. The construction of $\mathbf{f}_{t}$. In this subsection we define the families $\mathbf{f}_{t}$ for $0<t<1$ using quasiconformal surgery. Consider first a quadratic Blaschke product $h_{t}$ defined by

$$
h_{t}(z)=\frac{(t+2) z^{2}+t}{t z^{2}+t+2}, \quad t \in[0,1] .
$$

Then 0 is a $h_{t}$-critical point and $h_{t}(0)=t /(t+2)$. Moreover, for $t \in[0,1)$ the point

$$
w_{t}=\left(1-\sqrt{1-t^{2}}\right) / t
$$

is an attracting $h_{t}$-fixed point of multiplier $t$. Note that if $t \rightarrow 1^{-}$, then $h_{t} \rightarrow h_{1}$ uniformly in the spherical metric on $\widehat{\mathbb{C}}$.

For $t \in[0,1)$ let $D_{t} \subset \mathbb{D}$ be the open hyperbolic disc centred at $w_{t}$ such that $-\widehat{r} \in \partial D_{t}$ for $\widehat{r}$ from Definition 4.7. Note that $D_{t}$ is a Euclidean disc symmetric with respect to the real axis, containing the entire forward trajectory of the $h_{t}$-critical point 0 . Let

$$
W_{t}=h_{t}^{-1}\left(D_{t}\right) \cap \mathbb{D} \text {. }
$$

By definition, $W_{t}$ is a topological disc, $\partial W_{t}$ is a Jordan curve and $W_{t} \supset$ $\operatorname{cl} h_{t}\left(W_{t}\right)=\operatorname{cl} D_{t}$. Moreover, $h_{t}$ has degree two on $W_{t}$, is a degree two covering on the annulus $W_{t} \backslash \operatorname{cl} D_{t}$ and maps the segment $(0,1)$ into itself. See Fig. 9.

Consider now the map $N_{\sigma}$ for $\sigma \in \mathcal{U}^{\prime}$. Recall that the Riemann map $\varphi_{\sigma}^{u}$ from Corollary 4.9 conjugates $N_{\sigma}$ on $B_{\sigma}(u)$ to the Blaschke product $h_{\sigma}^{u}$ from (17) and $\Phi_{\sigma}^{u}$ are the Böttcher coordinates for $h_{\sigma}^{u}$ on $\mathbb{D}$ (see Subsection 4.1). Let

$$
D_{\sigma}=\left(\Phi_{\sigma}^{u}\right)^{-1}\left(\mathbb{D}_{\widehat{r}}\right), \quad W_{\sigma}=\left(\Phi_{\sigma}^{u}\right)^{-1}\left(\mathbb{D}_{\sqrt{\widehat{r}}}\right) .
$$




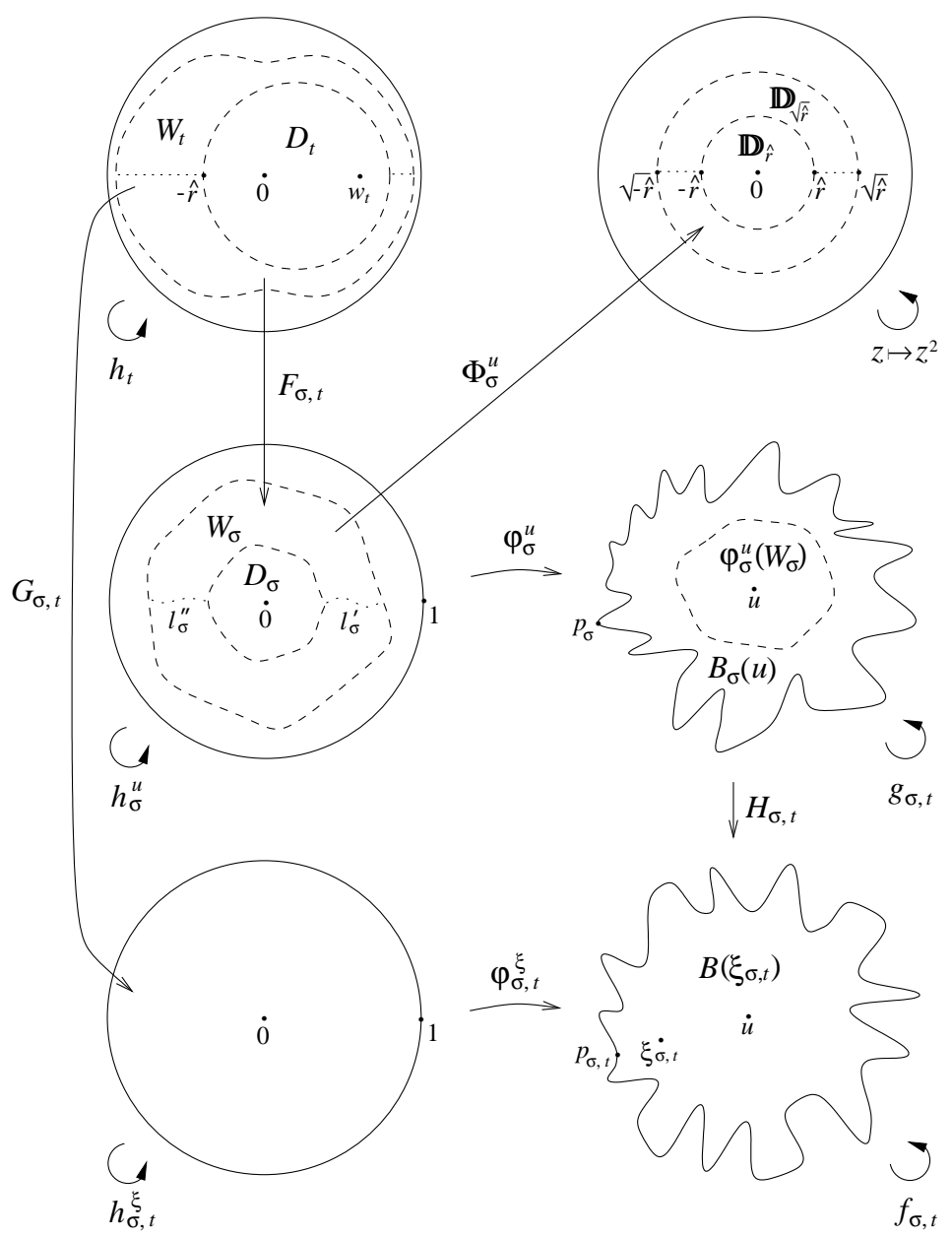

Fig. 9. The construction of $f_{\sigma, t}$

Then $W_{\sigma}$ is a component of $\left(h_{\sigma}^{u}\right)^{-1}\left(D_{\sigma}\right)$ such that $W_{\sigma}$ is a topological disc, $\partial W_{\sigma}$ is a Jordan curve, $W_{\sigma} \supset \operatorname{cl} D_{\sigma}, h_{\sigma}^{u}$ has degree two on $W_{\sigma}$ and is a degree two covering on the annulus $W_{\sigma} \backslash \operatorname{cl} D_{\sigma}$. Moreover, if $\sigma \in M_{N}$, then $D_{\sigma}=\mathbb{D}_{\widehat{r}}$ and $W_{\sigma}=\mathbb{D}_{\sqrt{\hat{r}}}$.

Let

$$
\ell_{\sigma}^{\prime}=\left(\Phi_{\sigma}^{u}\right)^{-1}([\widehat{r}, \sqrt{\widehat{r}}]), \quad \ell_{\sigma}^{\prime \prime}=\left(\Phi_{\sigma}^{u}\right)^{-1}([-\sqrt{\widehat{r}},-\widehat{r}])
$$

(see Fig. 9). Note that $\ell_{\sigma}^{\prime}=\gamma_{\sigma}^{\prime u} \cap \mathrm{cl} W_{\sigma}$ and $\ell_{\sigma}^{\prime \prime}=\gamma_{\sigma}^{\prime \prime u} \cap \mathrm{cl} W_{\sigma}$ for $\gamma_{\sigma}^{\prime u}, \gamma_{\sigma}^{\prime \prime u}$ from (24).

Now for $\sigma \in \mathcal{U}^{\prime}$ and $t \in[0,1)$ we define a $C^{1}$-diffeomorphism

$$
F_{\sigma, t}: \operatorname{cl} W_{t} \stackrel{\text { onto }}{\longrightarrow} \operatorname{cl} W_{\sigma}
$$


such that:

(a) $h_{\sigma}^{u} \circ F_{\sigma, t}=F_{\sigma, t} \circ h_{t}$ on $\partial W_{t}$,

(b) $F_{\sigma, t}$ maps biholomorphically $D_{t}$ onto $D_{\sigma}$ with $F_{\sigma, t}(0)=0$,

(c) $F_{\sigma, t}\left(\left(\operatorname{cl} W_{t} \backslash D_{t}\right) \cap(0,1)\right)=\ell_{\sigma}^{\prime}, F_{\sigma, t}\left(\left(\operatorname{cl} W_{t} \backslash D_{t}\right) \cap(-1,0)\right)=\ell_{\sigma}^{\prime \prime}$,

(d) $F_{\sigma, t}$ depends continuously on $\sigma, t$,

(e) $F_{\sigma, 0}$ is holomorphic for $\sigma \in \mathcal{U}^{\prime}$ and $F_{\sigma, 0}=$ id for $\sigma \in M_{N}$.

We define $F_{\sigma, t}$ as follows: on $\mathrm{cl} D_{t}$ let $F_{\sigma, t}$ be the Möbius map sending 0 and the two points from $\partial D_{t} \cap(-1,1)$ to $0,-\widehat{r}$ and $\widehat{r}$ respectively, composed with $\left(\Phi_{\sigma}^{u}\right)^{-1}$. Then the condition (b) is obviously satisfied. On each of the two segments of $\left(\operatorname{cl} W_{t} \backslash D_{t}\right) \cap(-1,1)$ let $F_{\sigma, t}$ be the composition of a suitable affine map with $\left(\Phi_{\sigma}^{u}\right)^{-1}$. Since both $h_{t}$ on $\partial W_{t}$ and $h_{\sigma}^{u}$ on $\partial W_{\sigma}$ are degree two coverings, the conditions (a) and (c) define $F_{\sigma, t}$ uniquely on $\partial W_{t}$. Then it is easy to extend $F_{\sigma, t} C^{1}$-diffeomorphically to both components of $\mathrm{cl} W_{t} \backslash$ $\left(D_{t} \cup(-1,1)\right)$ so that the conditions (d)-(e) are satisfied.

Let

$$
g_{\sigma, t}= \begin{cases}N_{\sigma} & \text { on } \widehat{\mathbb{C}} \backslash \varphi_{\sigma}^{u}\left(W_{\sigma}\right), \\ \varphi_{\sigma}^{u} \circ F_{\sigma, t} \circ h_{t} \circ F_{\sigma, t}^{-1} \circ\left(\varphi_{\sigma}^{u}\right)^{-1} & \text { on } \varphi_{\sigma}^{u}\left(W_{\sigma}\right) .\end{cases}
$$

By definition, $g_{\sigma, t}$ is a $C^{1}$ cubic branched cover of $\widehat{\mathbb{C}}$, holomorphic on $\widehat{\mathbb{C}} \backslash \operatorname{cl} \varphi_{\sigma}^{u}\left(W_{\sigma}\right)$ and $g_{\sigma, t}\left(\varphi_{\sigma}^{u}\left(W_{\sigma}\right)\right)$. Moreover, $\operatorname{cl} g_{\sigma, t}\left(\varphi_{\sigma}^{u}\left(W_{\sigma}\right)\right) \subset \varphi_{\sigma}^{u}\left(W_{\sigma}\right)$, the function $(\sigma, t) \mapsto g_{\sigma, t}$ is continuous, and by the condition (e) and the uniqueness of the Böttcher coordinates, we have $g_{\sigma, 0}=N_{\sigma}$.

Now we define a continuous family of $g_{\sigma, t}$-invariant conformal structures $\mu_{\sigma, t}$ on $\widehat{\mathbb{C}}$ setting

$$
\mu_{\sigma, t}= \begin{cases}\mu_{0} & \text { on } g_{\sigma, t}\left(\varphi_{\sigma}^{u}\left(W_{\sigma}\right)\right), \\ \left(g_{\sigma, t}^{n}\right)^{*}\left(\mu_{0}\right) & \text { on } g_{\sigma, t}^{-n}\left(g_{\sigma, t}\left(\varphi_{\sigma}^{u}\left(W_{\sigma}\right)\right)\right), \\ \mu_{0} & \text { else, }\end{cases}
$$

where $\mu_{0}$ is the standard structure. Note that $\mu_{\sigma, t} \in L^{\infty}$, because for every $z \in \widehat{\mathbb{C}}$ its forward trajectory under $g_{\sigma, t}$ hits at most twice the closed annulus $\operatorname{cl} \varphi_{\sigma}^{u}\left(W_{\sigma}\right) \backslash g_{\sigma, t}\left(\varphi_{\sigma}^{u}\left(W_{\sigma}\right)\right)$, where the map is not holomorphic. By the measurable Riemann theorem (see [DH2]), there exists a continuous family of quasiconformal homeomorphisms $H_{\sigma, t}$ of $\widehat{\mathbb{C}}$ such that $H_{\sigma, t}(\zeta)=\zeta$ for $\zeta \in\{0,1, \infty\}, H_{\sigma, t} \circ g_{\sigma, t} \circ H_{\sigma, t}^{-1}$ is rational and $H_{\sigma, 0}=$ id. Moreover, $H_{\sigma, t}$ is holomorphic on $\widehat{\mathbb{C}} \backslash \operatorname{cl} \bigcup_{n=0}^{\infty} g_{\sigma, t}^{-n}\left(\varphi_{\sigma}^{u}\left(W_{\sigma}\right)\right)$ and $g_{\sigma, t}\left(\varphi_{\sigma}^{u}\left(W_{\sigma}\right)\right)$. It is easy to check that $H_{\sigma, t} \circ g_{\sigma, t} \circ H_{\sigma, t}^{-1}=f_{a(\sigma, t), b(\sigma, t)}$ for some continuous functions $a(\sigma, t), b(\sigma, t)$. For simplicity, write $f_{\sigma, t}$ for $f_{a(\sigma, t), b(\sigma, t)}$. Then $f_{\sigma, 0}=N_{\sigma}$ because $H_{\sigma, 0}=$ id. Moreover, since $g_{\sigma, t}$ has an attracting fixed point of multiplier $t$ in $g_{\sigma, t}\left(\varphi_{\sigma}^{u}\left(W_{\sigma}\right)\right)$, we have $(a(\sigma, t), b(\sigma, t)) \in \operatorname{Fix}(t)$. Let

$$
U_{\sigma, t}=H_{\sigma, t}\left(U_{\sigma}^{\prime}\right) \text {. }
$$


Note that by the definitions of $U_{\sigma}^{\prime}$ and $g_{\sigma, t}$, we have $g_{\sigma, t}^{k}=N_{\sigma}^{k}$ on $U_{\sigma}^{\prime}$. Hence, $U_{\sigma, t}$ is a topological disc containing 1 and the map $\left.f_{\sigma, t}^{k}\right|_{U_{\sigma, t}}$ is quadratic-like and hybrid equivalent to $\left.N_{\sigma}^{k}\right|_{U_{\sigma}^{\prime}}$. Moreover,

$$
\partial f_{\sigma, t}^{k}\left(U_{\sigma, t}\right)=\operatorname{cl}\left(H_{\sigma, t}\left(\varphi_{\sigma}^{u}\left(\Gamma_{\sigma}^{u}\right)\right) \cup H_{\sigma, t}\left(\varphi_{\sigma}^{\infty}\left(\Gamma_{\sigma}^{\infty}\right)\right)\right)
$$

for $\Gamma_{\sigma}^{u}, \Gamma_{\sigma}^{\infty}$ from (24).

Let $\xi_{\sigma, t}$ be the attracting $f_{\sigma, t}$-fixed point. Denote by $B\left(\xi_{\sigma, t}\right)$ its immediate basin of attraction and let $p_{\sigma, t}$ be the unique repelling $f_{\sigma, t}$-fixed point. By construction, $u \in B\left(\xi_{\sigma, t}\right)$. Note that the boundary of $f_{\sigma, t}^{k}\left(U_{\sigma, t}\right)$ in $B\left(\xi_{\sigma, t}\right)$ near $p_{\sigma, t}$ forms a curve ending at $p_{\sigma, t}$. Let

$$
\varphi_{\sigma, t}^{\xi}: \mathbb{D} \rightarrow B\left(\xi_{\sigma, t}\right)
$$

be the unique Riemann mapping such that $\varphi_{\sigma, t}^{\xi}(0)=u$ and $\left(\varphi_{\sigma, t}^{\xi}\right)^{-1}$ maps this curve to a curve ending at 1 . This map extends to $\partial \mathbb{D}$, because $\partial B\left(\xi_{\sigma, t}\right)$ $=H_{\sigma, t}\left(\partial B_{\sigma}(u)\right)$ is locally connected. In particular, this implies $\varphi_{\sigma, t}^{\xi}(1)=p_{\sigma, t}$.

The map $\varphi_{\sigma, t}^{\xi}$ conjugates $f_{\sigma, t}$ on $B\left(\xi_{\sigma, t}\right)$ to some quadratic or cubic Blaschke product $h_{\sigma, t}^{\xi}$ with an attracting fixed point of multiplier $t$, a critical point at 0 and a fixed point at 1 . If $1 \notin B\left(\xi_{\sigma, t}\right)$, then $\operatorname{deg} h_{\sigma, t}^{\xi}=2$ and it is easy to check that in this case $h_{\sigma, t}^{\xi}=h_{t}$ for $h_{t}$ from (26). If $1 \in B\left(\xi_{\sigma, t}\right)$, then $\operatorname{deg} h_{\sigma, t}^{\xi}=3$.

Let

$$
\varphi_{\sigma, t}^{\infty}=H_{\sigma, t} \circ \varphi_{\sigma}^{\infty} .
$$

Since $H_{\sigma, t}$ is holomorphic on $B(\infty)$ for $N_{\sigma}$, the map $\varphi_{\sigma, t}^{\infty}$ is a Riemann mapping from $\mathbb{D}$ onto $B(\infty)$ for $f_{\sigma, t}$ such that $\varphi_{\sigma, t}^{\infty}(0)=\infty$ and $\varphi_{\sigma, t}^{\infty}(1)=$ $p_{\sigma, t}$. Moreover, $\varphi_{\sigma, t}^{\infty}$ conjugates $f_{\sigma, t}$ on $B(\infty)$ to $h_{\sigma}^{\infty}$ from (17).

By Lemma 2.3 and Corollary 4.9, it is easy to see that for $\zeta \in\{\xi, \infty\}$ the map $\varphi_{\sigma, t}^{\zeta}$ depends continuously on $\sigma, t$ in the almost uniform convergence topology.

Let

$$
G_{\sigma, t}=\left(\varphi_{\sigma, t}^{\xi}\right)^{-1} \circ H_{\sigma, t} \circ \varphi_{\sigma}^{u} \circ F_{\sigma, t} .
$$

Then $G_{\sigma, t}$ is a quasiconformal homeomorphism on $W_{t}$ and conjugates $h_{t}$ to $h_{\sigma, t}^{\xi}$ (see Fig. 9). By definition, $G_{\sigma, t}$ is holomorphic on $D_{t}$ and since it conjugates two holomorphic maps, it is easy to see that in fact it is holomorphic on $W_{t}$.

Now we show that if $\left(a\left(\sigma_{1}, t_{1}\right), b\left(\sigma_{1}, t_{1}\right)\right)=\left(a\left(\sigma_{2}, t_{2}\right), b\left(\sigma_{2}, t_{2}\right)\right)$, then $U_{\sigma_{1}, t_{1}}=U_{\sigma_{2}, t_{2}}$. It is obvious that we must have $t_{1}=t_{2}=t$. Hence, $G_{\sigma_{1}, t}^{-1} \circ G_{\sigma_{2}, t}$ conjugates holomorphically $h_{t}$ to $h_{t}$ on $W_{t}$. Since $W_{t}$ contains the infinite forward orbit of the $h_{t}$-critical point 0 , it follows that 
$G_{\sigma_{1}, t}^{-1} \circ G_{\sigma_{2}, t}=$ id on $W_{t}$. This together with the definition of $U_{\sigma, t}$ easily implies $U_{\sigma_{1}, t}=U_{\sigma_{2}, t}$.

Note that if $1 \notin B\left(\xi_{\sigma, t}\right)$ (which is equivalent to $1 \notin B_{\sigma}(u)$ ), then $G_{\sigma, t}$ conjugates $h_{t}$ to $h_{t}$, so $G_{\sigma, t}=\mathrm{id}$. Moreover, $N_{\sigma}^{k}\left(U_{\sigma}^{\prime}\right) \cap B_{\sigma}(u)=\varphi_{\sigma}^{u}\left(\mathbb{D}^{+} \backslash \mathrm{cl} \mathbb{D}_{\widehat{r}}\right)$ in this case. Hence, by the definition of $F_{\sigma, t}$,

$$
\text { If } 1 \notin B\left(\xi_{\sigma, t}\right) \text {, then } f_{\sigma, t}^{k}\left(U_{\sigma, t}\right) \cap B\left(\xi_{\sigma, t}\right)=\varphi_{\sigma, t}^{\xi}\left(\mathbb{D}^{+} \backslash \operatorname{cl} D_{t}\right) .
$$

Let

$$
Q_{t}(\sigma)=(a(\sigma, t), b(\sigma, t)), \quad \sigma \in \mathcal{U}^{\prime}, t \in[0,1) .
$$

Lemma 4.11. For every $t \in[0,1)$ and $\left(a_{0}, b_{0}\right) \in Q_{t}\left(\mathcal{U}^{\prime}\right)$ there exists an open neighbourhood $\mathcal{V}$ of $\left(a_{0}, b_{0}\right)$ in $\mathbb{C} \times \mathbb{C}$ such that $\operatorname{Fix}(t) \cap \mathcal{V}$ is a onedimensional complex manifold.

Proof. Note that for $(a, b)$ near $\left(a_{0}, b_{0}\right)$ all the critical and fixed points for the map $f_{a, b}$ are simple. By the implicit function theorem, for $(a, b)$ close to $\left(a_{0}, b_{0}\right)$ there exists a holomorphic map $(a, b) \mapsto \xi(a, b) \neq 0, \infty$ such that $\xi(a, b)$ is an attracting $f_{a, b}$-fixed point and $\xi\left(a_{0}, b_{0}\right)$ has multiplier $t$. Then the map $z \mapsto f_{a, b}(\xi(a, b) z) / \xi(a, b)$ has supersinks at $0, \infty$ and a fixed point at 1 . It is easy to check that such a map has the form

$$
\widetilde{f}_{A, B}(z)=z^{2} \frac{z+A+B-1}{A z+B}, \quad A \neq 1, B \neq 0, A+B \neq 0,
$$

for $(A, B)$ depending holomorphically on $(a, b)$. Since $\widetilde{f}_{A, B}$ has only simple critical points, in the similar way we can define locally a holomorphic mapping $(A, B) \mapsto(a, b)$, which is the inverse of $(a, b) \mapsto(A, B)$. It follows that in fact the map $(a, b) \mapsto(A, B)$ is biholomorphic in a small neighbourhood $\mathcal{V} \subset \mathbb{C} \times \mathbb{C}$ of $\left(a_{0}, b_{0}\right)$. But $\tilde{f}_{A, B}^{\prime}(1)=(A+2 B+1) /(A+B)$, so in the parameterization $(A, B)$ the set $\operatorname{Fix}(t)$ is a surface described by

$$
B=\frac{A t-A-1}{2-t} .
$$

Hence, $\operatorname{Fix}(t) \cap \mathcal{V}$ is a one-dimensional complex manifold.

Remark. Actually, one can prove that for every $\varrho \in \mathbb{C}$ the entire set $\operatorname{Fix}(\varrho)$ is a manifold.

Define

$$
M_{t}=Q_{t}\left(M_{N}\right) .
$$

Note that $Q_{0}=\mathrm{id}$, so $M_{0}=M_{N}$. Moreover, $Q_{t}: \mathcal{U}^{\prime} \rightarrow \operatorname{Fix}(t)$ is continuous and $\left.f_{Q_{t}(\sigma)}^{k}\right|_{U_{\sigma, t}}=\left.f_{\sigma, t}^{k}\right|_{U_{\sigma, t}}$ is hybrid equivalent to $\left.N_{\sigma}^{k}\right|_{U_{\sigma}^{\prime}}$. By the uniqueness of hybrid equivalence and the compactness of $M_{N}$, the map $\left.Q_{t}\right|_{M_{N}}: M_{N} \rightarrow M_{t}$ is a homeomorphism and $Q_{t}\left(M_{N}\right) \cap Q_{t}\left(\mathcal{U}^{\prime} \backslash M_{N}\right)=\emptyset$.

Lemma 4.12. $M_{t} \subset \operatorname{int}_{\mathrm{Fix}(t)} Q_{t}\left(\mathcal{U}^{\prime}\right)$ for every $t \in[0,1)$. 
Proof. Choose a point $\sigma_{0} \in \operatorname{int}_{\mathcal{U}^{\prime}} M_{N}$ and Jordan curves $\gamma_{0}, \gamma_{1}$ such that $\gamma_{0} \subset$ int $_{\mathcal{U}^{\prime}} M_{N} \backslash\left\{\sigma_{0}\right\}, \gamma_{1} \subset \mathcal{U}^{\prime} \backslash M_{N}, \gamma_{0}$ and $\gamma_{1}$ are homotopic in $\mathcal{U}^{\prime} \backslash\left\{\sigma_{0}\right\}$ and the index of $\gamma_{i}$ with respect to $\sigma_{0}$ is equal to 1 for $i=0,1$. By Lemma 4.11 and the fact that $\left.Q_{t}\right|_{M_{N}}$ is a homeomorphism, using topological properties of surfaces we can find a topological disc $\mathcal{W} \subset \operatorname{Fix}(t)$ containing $M_{t}$. The index of $Q_{t} \circ \gamma_{0}$ with respect to $Q_{t}\left(\sigma_{0}\right)$ is equal to 1 , because $Q_{t}$ is a holomorphic bijection on $\operatorname{int}_{\mathcal{U}^{\prime}} M_{N}$ (see Theorem 3.3). Moreover, since $Q_{t}$ is continuous, $\left.Q_{t}\right|_{M_{N}}$ is a homeomorphism and $Q_{t}\left(M_{N}\right) \cap Q_{t}\left(\mathcal{U}^{\prime} \backslash M_{N}\right)=\emptyset$, if we take $\gamma_{1}$ sufficiently close to $M_{N}$, then $Q_{t} \circ \gamma_{0}$ and $Q_{t} \circ \gamma_{1}$ are homotopic in $\mathcal{W} \backslash Q_{t}\left(\sigma_{0}\right)$. Hence, the index of $Q_{t} \circ \gamma_{1}$ with respect to $Q_{t}\left(\sigma_{0}\right)$ is equal to 1 .

Let $C_{t}$ be the component of $\mathcal{W} \backslash Q_{t}\left(\gamma_{1}\right)$ containing $Q_{t}\left(\sigma_{0}\right)$. Then $C_{t}$ is a topological disc in $\mathcal{W}$ and $M_{t} \subset C_{t}$. We show that $C_{t} \subset Q_{t}\left(\mathcal{U}^{\prime}\right)$. Suppose the converse and take a point $(a, b) \in C_{t} \backslash Q_{t}\left(\mathcal{U}^{\prime}\right)$. Then the index of $Q_{t} \circ \gamma_{0}$ with respect to $(a, b)$ is equal to 0 and the curves $Q_{t} \circ \gamma_{0}, Q_{t} \circ \gamma_{1}$ are homotopic in $\mathcal{W} \backslash(a, b)$, so the index of $Q_{t} \circ \gamma_{1}$ with respect to $(a, b)$ is equal to 0 . But on the other hand, both points $Q_{t}\left(\sigma_{0}\right)$ and $(a, b)$ are in $C_{t}$, which implies that the indices of $Q_{t} \circ \gamma_{1}$ with respect to $(a, b)$ and $Q_{t}\left(\sigma_{0}\right)$ must be equal. This is a contradiction. Therefore, $C_{t} \subset Q_{t}\left(\mathcal{U}^{\prime}\right)$, so $M_{t} \subset \operatorname{int}_{\text {Fix }(t)} Q_{t}\left(\mathcal{U}^{\prime}\right)$.

Using Lemmas 4.11 and 4.12 and the fact that $Q_{t}$ is continuous and depends continuously on $t \in[0,1)$ we can find topological $\operatorname{discs} \mathcal{U}_{t} \subset \operatorname{Fix}(t)$, $t \in[0,1)$, such that $M_{N} \subset \mathcal{U}_{0} \subset \mathcal{U}^{\prime}, M_{t} \subset \mathcal{U}_{t} \subset \operatorname{int}_{\text {Fix }(t)} Q_{t}\left(\mathcal{U}^{\prime}\right)$ for $t \in(0,1)$ and $\operatorname{cl} \mathcal{U}_{t}$ depends continuously on $t \in[0,1)$ in the Hausdorff metric. Then there exists a homeomorphism from $\mathbb{D} \times[0,1)$ onto $\bigcup_{t \in[0,1)} \mathcal{U}_{t}$ mapping holomorphically $\mathbb{D} \times\{t\}$ onto $\mathcal{U}_{t}$ for each $t$. Note that $\operatorname{cl} U_{\sigma, t}$ depends continuously on $\sigma \in \mathcal{U}^{\prime}, t \in[0,1)$ in the Hausdorff metric. These facts together with Theorem 3.3 and Proposition 3.5 give

Corollary 4.13. Define $U_{a, b}=U_{\sigma, t}$ for $(a, b)=(a(\sigma, t), b(\sigma, t)), \sigma \in$ $\mathcal{U}^{\prime}, t \in[0,1)$. Then $\left\{\left.f_{a, b}^{k}\right|_{U_{a, b}}\right\}_{(a, b) \in \mathcal{U}_{t}}$ is a Mandelbrot-like family with the Mandelbrot-like set $M_{t}$ and $\left\{\left\{\left.f_{a, b}^{k}\right|_{U_{a, b}}\right\}_{(a, b) \in \mathcal{U}_{t}}\right\}_{t \in\left[0, t_{0}\right]}$ is a continuous path of Mandelbrot-like families for every $t_{0} \in[0,1)$.

4.3. The construction of $\mathbf{f}_{1}$. Consider a sequence $\left(a\left(\sigma_{n}, t_{n}\right), b\left(\sigma_{n}, t_{n}\right)\right)_{n>0}$ for $\sigma_{n} \in \mathcal{U}^{\prime}$ and $t_{n} \in[0,1)$ such that $t_{n} \rightarrow 1^{-}$. Passing to a subsequence we can assume it converges to some $(a, b) \in \widehat{\mathbb{C}} \times \widehat{\mathbb{C}}$. It is easy to see that $(a, b)$ is either in $\operatorname{Fix}(1)$ or in Sing. Now we show that if $\sigma_{n} \in M_{N}$, then the case $(a, b) \in$ Sing is not possible. In other words, we prove

Proposition 4.14. For every $(a, b) \in \widehat{\mathbb{C}} \times \widehat{\mathbb{C}}$, if $\left(a\left(\sigma_{n}, t_{n}\right), b\left(\sigma_{n}, t_{n}\right)\right) \rightarrow$ $(a, b)$ as $n \rightarrow \infty$ for $\sigma_{n} \in M_{N}, t_{n} \rightarrow 1^{-}$, then $(a, b) \notin$ Sing.

Proof. Recall that by the definition of $U_{\sigma, t}$, if $\sigma_{n} \in M_{N}$, then for the map $f_{\sigma_{n}, t_{n}}$ we have $u \in B\left(\xi_{\sigma_{n}, t_{n}}\right)$ and 1 is not in the basins of $0, \infty, \xi_{\sigma_{n}, t_{n}}$. 
We consider several cases. Suppose first $a=0, b \neq \infty$. By (1), there exists $r>0$ such that for sufficiently large $n$ we have $\operatorname{cl} f_{\sigma_{n}, t_{n}}\left(\mathbb{D}_{r}\right) \subset \mathbb{D}_{r}$, so $\mathbb{D}_{r} \subset B(0)$ for $f_{\sigma_{n}, t_{n}}$. But $f_{\sigma_{n}, t_{n}}(1)=a\left(\sigma_{n}, t_{n}\right) \in \mathbb{D}_{r}$ for large $n$. This is a contradiction.

Suppose now $a=0, b=\infty$. Let $b_{n}=b\left(\sigma_{n}, t_{n}\right)$. If $|z|<1 /\left|2 b_{n}\right|$, then for large $n$ we have $\left|\left(2-b_{n}\right) z-1\right|>$ const $>0$, so by $(1), \operatorname{cl} f_{\sigma_{n}, t_{n}}\left(\mathbb{D}_{1 /\left|2 b_{n}\right|}\right) \subset$ $\mathbb{D}_{1 /\left|2 b_{n}\right|}$ and $\mathbb{D}_{1 /\left|2 b_{n}\right|} \subset B(0)$ for $f_{\sigma_{n}, t_{n}}$. On the other hand, by $(2), f_{\sigma_{n}, t_{n}}(u)$ $\in \mathbb{D}_{1 /\left|2 b_{n}\right|}$ for large $n$. Hence, $u$ is in the basin of 0 for $f_{\sigma_{n}, t_{n}}$, which is impossible.

We have proved the case $a=0$. Since $f_{1 / a, 1 / b}$ is conjugate to $f_{a, b}$ by $z \mapsto 1 / z$, this covers also the case $a=\infty$.

Suppose $a \neq 0,1, \infty, b=1$. By Lemma I.2.2, $f_{\sigma_{n}, t_{n}}(z)$ tends to $a z^{2}$ uniformly in the spherical metric outside a small neighbourhood of 1 . The map $a z^{2}$ has a fixed point $1 / a \neq 1$ of multiplier 2 . Hence, the map $f_{\sigma_{n}, t_{n}}$ for large $n$ has a fixed point of multiplier close to 2 . By (3), the other fixed point cannot be attracting, which is a contradiction.

Suppose now $a=b=1$. By Lemma I.2.2, $f_{\sigma_{n}, t_{n}}$ tends to the polynomial $z \mapsto z^{2}$ almost uniformly in the spherical metric on $\widehat{\mathbb{C}} \backslash\{1\}$. Let $\varphi_{n}^{\zeta}=\varphi_{\sigma_{n}, t_{n}}^{\zeta}$ for $\zeta \in\{0, \infty\}$. It is clear that the map $f_{\sigma_{n}, t_{n}}$ on $B(\zeta)$ satisfies the conditions of Lemma 2.3 with $\varphi_{n}^{\zeta}$ instead of $\psi_{n}$ (cf. Corollary 4.9). By this lemma, $\varphi_{n}^{0}(z) \rightarrow z$ and $\varphi_{n}^{\infty}(z) \rightarrow 1 / z$ almost uniformly on $\mathbb{D}$. (Note that in this case we have $h_{n}(z)=h(z)=z^{2}$ for every $n$.) Let $\gamma:[0, \infty) \rightarrow \mathbb{D}$ be the curve parameterizing the segment $[1 / 2,1)$ such that $\gamma(0)=1 / 2, \gamma(1)=\sqrt{1 / 2}, \gamma$ is affine on $[0,1]$ and $(\gamma(s))^{2}=\gamma(s-1)$ for $s>1$. Then the assumptions of the case (a) of Lemma 2.4 are fulfilled for $\gamma_{n}=\gamma$ and $\varphi_{n}^{\zeta}$ instead of $\psi_{n}$. Using this lemma, in the same way as in the proof of (23) we show that $\varphi_{n}^{0}((-1,-\sqrt{1 / 2}])$ and $\varphi_{n}^{\infty}((-1,-\sqrt{1 / 2}])$ land at the same point near -1 (we use the fact that $z \mapsto z^{2}$ sends -1 to 1 and $f_{\sigma_{n}, t_{n}}(z) \rightarrow z^{2}$ uniformly near -1$)$. In the language of internal rays this means $l_{0}(1 / 2)=l_{\infty}(1 / 2)$ for $f_{\sigma_{n}, t_{n}}$. Hence, by the definition of $f_{\sigma, t}$, we have $l_{0}(1 / 2)=l_{\infty}(1 / 2)$ for $N_{\sigma_{n}}$. But this is impossible by the combinatorics of $N_{\sigma}$ for $\sigma \in M_{N}$ (see e.g. Lemma 4.6 and Subsection I.4.4). In this way we have shown that the case $a=b=1$ does not hold.

We are left with the case $a \neq 0, \infty, b=0, \infty$. If $b=\infty$, then $f_{\sigma_{n}, t_{n}}$ tends to $g(z)=a z(2-z)$ uniformly outside a small neighbourhood of 0 . The polynomial $g$ has a fixed point $2-1 / a$ of multiplier $2-2 a$. Suppose $a \neq 1 / 2$. Then $2-1 / a \neq 0$, so by the uniform convergence near $2-1 / a$, we have $\xi_{\sigma_{n}, t_{n}} \rightarrow 2-1 / a$ and $2-2 a=g^{\prime}(2-1 / a)=\lim _{n} f_{\sigma_{n}, t_{n}}^{\prime}\left(\xi_{\sigma_{n}, t_{n}}\right)=1$. This leads to a contradiction. Hence, if $b=\infty$, then $a=1 / 2$. Similarly, if $b=0$, then $a=2$. We conclude that the only possibilities are: $(a, b)=(1 / 2, \infty),(2,0)$.

Suppose $(a, b)=(1 / 2, \infty)$. To make use of Lemma 2.4 , we change the coordinates in $\widehat{\mathbb{C}}$ by a suitable Möbius map. More precisely, let $f_{n}=H \circ$ 
$f_{\sigma_{n}, t_{n}} \circ H^{-1}$, where $H$ exchanges 0 and $\infty$ and maps 1 to $u$ (this is the map $h_{2}$ from Lemma I.3.2). Note that the $f_{n}$-critical point 1 is in the immediate basin of an attracting $f_{n}$-fixed point $\xi_{n}$ of multiplier $t_{n}$ and the fourth $f_{n^{-}}$ critical point $u_{n}$ is in the filled-in Julia set of $f_{n}^{k}$ as a quadratic-like map on some topological disc. Moreover, by Lemmas I.2.2 and I.3.2, $f_{n}$ tends to the quadratic polynomial $g(z)=z-z^{2} / 2$ almost uniformly in the spherical metric on $\widehat{\mathbb{C}} \backslash\{0\}$. Note that $g$ is a quadratic polynomial with a parabolic fixed point at 0 of multiplier 1 , a critical point at 1 and is conformally conjugate to $z \mapsto z^{2}+1 / 4$.

Let $\varphi_{n}^{\zeta}=H \circ \varphi_{\sigma_{n}, t_{n}}^{\zeta}$ for $\zeta \in\{\infty, \xi\}$. Note that $\varphi_{n}^{\infty}$ conjugates $f_{n}$ to $z \mapsto z^{2}$ and $\varphi_{n}^{\xi}$ conjugates $f_{n}$ to $h_{t_{n}}$ from (26). It is easy to see that $f_{n}$ on $B(\infty)$ (resp. $B\left(\xi_{n}\right)$ ) satisfies the assumptions of Lemma 2.3 with $\varphi_{n}^{\infty}$ (resp. $\left.\varphi_{n}^{\xi}\right)$ instead of $\psi_{n}$. Using this lemma, we obtain $\varphi_{n}^{\infty} \rightarrow \varphi^{\infty}$ and $\varphi_{n}^{\xi} \rightarrow \varphi^{0}$ almost uniformly on $\mathbb{D}$ for suitable Riemann maps $\varphi^{0}$ onto $B(0)$ and $\varphi^{\infty}$ onto $B(\infty)$ for the polynomial $g$. Moreover, $h_{t_{n}} \rightarrow h_{1}$ for $h_{1}$ from (26).

Let $\gamma^{\infty}$ be the curve $\gamma$ defined in the proof of the case $a=b=1$. Then the conditions of the case (a) of Lemma 2.4 are satisfied for $\gamma_{n}=$ $\gamma^{\infty}, h_{n}(z)=z^{2}$ and $\varphi_{n}^{\infty}$ instead of $\psi_{n}$. Note that the segment $[1 / 2,1)$ is $h_{t_{n}}$-invariant, contains the attracting $h_{t_{n}}$-fixed point $s_{t_{n}}$ and lands at the repelling $h_{t_{n}}$-fixed point 1 . It is easy to see that we can define a curve $\gamma^{\xi_{n}}$ parameterizing $[1 / 2,1)$ in such a way that the conditions of the case (b) of this lemma are satisfied for $\gamma_{n}=\gamma^{\xi_{n}}, h_{n}=h_{t_{n}}$ and $\varphi_{n}^{\xi}$ instead of $\psi_{n}$. In the same way as in the case $a=b=1$ we show that $\varphi_{n}^{\infty}((-1,-\sqrt{1 / 2}])$ and $\varphi_{n}^{\xi}((-1,-\sqrt{1 / 2}])$ land at the same point near 2 . Recall that defining $f_{n}$ we changed the coordinates exchanging $0, \infty$ and $1, u$. Hence, for the map $f_{\sigma_{n}, t_{n}}$ we obtain $l_{0}(1 / 2) \in \mathrm{cl} B\left(\xi_{t_{n}}\right)$. This is again impossible by the definition of $f_{\sigma, t}$ and the combinatorics of $N_{\sigma}$ for $\sigma \in M_{N}$.

We are left with the case $(a, b)=(2,0)$. As previously, we change the coordinates in $\widehat{\mathbb{C}}$ setting $f_{n}=H \circ f_{\sigma_{n}, t_{n}} \circ H^{-1}$ for the Möbius map $H$ fixing 0 and $\infty$ and sending 1 to $u$ (then $H=h_{1} \circ h_{2}$ for $h_{1}, h_{2}$ from Lemma I.3.2). Again, the $f_{n}$-critical point 1 is in the immediate basin $B\left(\xi_{n}\right)$ of an invariant $f_{n}$-fixed point $\xi_{n}$ and the fourth $f_{n}$-critical point $u_{n}$ is in the suitable filled-in Julia set of a quadratic-like map. Moreover, $f_{n}$ tends to $g$ almost uniformly in the spherical metric on $\widehat{\mathbb{C}} \backslash\{0\}$ for $g$ as in the case $(a, b)=(1 / 2, \infty)$.

Define $\varphi_{n}^{\infty}, \varphi_{n}^{\xi}, \gamma^{\infty}, \gamma^{\xi_{n}}$ for $f_{n}$ as in the case $(a, b)=(1 / 2, \infty)$. In the same way as in the proof of (25) we find small $\varepsilon, \varepsilon^{\prime}>0$ and $s_{0}>0$ such that for large $n$ we have $\varphi_{n}^{\infty}\left(\gamma^{\infty}\left(\left[s_{0}, \infty\right)\right)\right), \varphi_{n}^{\xi}\left(\gamma^{\xi_{n}}\left(\left[s_{0}, \infty\right)\right)\right) \subset \mathbb{D}_{\varepsilon}$ and $f_{n}^{k-1}\left(\mathbb{D}_{\varepsilon^{\prime}}(x)\right), f_{n}^{k}\left(\mathbb{D}_{\varepsilon^{\prime}}(y)\right) \supset \mathbb{D}_{\varepsilon}$, where $x=l_{\infty}(1-2 \beta)$ and $y=l_{\infty}(1-2 \delta)$ for the polynomial $g$ and the angles $\beta, \delta$ from the definition of $U_{\sigma}^{\prime}$. Let $U_{n}$ be the topological disc $U_{\sigma_{n}, t_{n}}$ in the new coordinates. By the above facts and the definition of $U_{\sigma, t}$, we have 


$$
\partial f_{n}\left(U_{n}\right) \subset \mathbb{D}_{\varepsilon^{\prime}}(x) \cup \mathbb{D}_{\varepsilon^{\prime}}(y) \cup \varphi_{n}^{\infty}\left(\mathbb{D}_{r}\right) \cup \varphi_{n}^{\xi}\left(\mathbb{D}_{r}\right)
$$

for some fixed $r<1$. Since $x, y \neq 0$ and $0 \notin B(0) \cup B(\infty)$ for $g$, taking sufficiently small $\varepsilon^{\prime}$ and using the uniform convergence of $\varphi_{n}^{\infty}$ and $\varphi_{n}^{\xi}$ on $\mathbb{D}_{r}$ we get $\left|f_{n}\left(u_{n}\right)\right|>c$ for some fixed constant $c>0$ and large $n$. But this is a contradiction, because $f_{n} \rightarrow g$, so by (2), we have $f_{n}\left(u_{n}\right) \rightarrow 0$. This ends the proof.

REMARK. Proposition 4.14 completes the proof of Theorem I.4.20 showing that the curve $\gamma$ from this theorem does not land at a singular parameter $\lambda$.

Definition 4.15. Let $M_{1}$ be the set of all points $(a, b) \in \widehat{\mathbb{C}} \times \widehat{\mathbb{C}}$ such that $(a, b)$ is a limit point of a sequence $\left(a\left(\sigma_{n}, t_{n}\right), b\left(\sigma_{n}, t_{n}\right)\right)_{n>0}$, where $\sigma_{n} \in M_{N}$, $t_{n} \in[0,1)$ and $t_{n} \rightarrow 1^{-}$.

By Proposition 4.14, $M_{1}$ is a subset of Fix(1).

Definition 4.16. Let $\widetilde{\mathcal{U}}_{1}$ be the set of all points $(a, b) \in \widehat{\mathbb{C}} \times \widehat{\mathbb{C}}$ such that $\operatorname{dist}\left((a, b), M_{1}\right)<\varepsilon_{1}$ for a fixed small $\varepsilon_{1}>0$ and $(a, b)$ is a limit point of a sequence $\left(a\left(\sigma_{n}, t_{n}\right), b\left(\sigma_{n}, t_{n}\right)\right)_{n>0}$, where $\sigma_{n} \in \mathcal{U}^{\prime}, t_{n} \in[0,1)$ and $t_{n} \rightarrow 1^{-}$.

By (1), it is easy to check that $\operatorname{Fix}(1)$ is described by the equation

$$
(2 b-1)^{2} a^{2}-2\left(2 b^{2}-3 b+2\right) a+(b-2)^{2}=0,
$$

which for $(a, b) \notin$ Sing gives locally two surfaces $a=a_{1}(b)$ and $a=a_{2}(b)$. Hence, Fix(1) is a one-dimensional complex manifold. Moreover, $M_{1}$ is compact by definition, so for sufficiently small $\varepsilon_{1}$ we have

$$
M_{1} \subset \widetilde{\mathcal{U}}_{1} \subset \operatorname{Fix}(1) .
$$

Thus, if $\left(a\left(\sigma_{n}, t_{n}\right), b\left(\sigma_{n}, t_{n}\right)\right) \rightarrow(a, b) \in \widetilde{\mathcal{U}}_{1}$, then $f_{\sigma_{n}, t_{n}} \rightarrow f_{a, b}$ uniformly in the spherical metric on $\widehat{\mathbb{C}}$.

Before defining the family $\mathbf{f}_{1}$ we prove the following technical lemma similar to Lemma 4.8.

LEMma 4.17. There exists $\varepsilon_{0}>0$ such that for every $\sigma_{n} \in \mathcal{U}^{\prime}, t_{n} \rightarrow 1^{-}$, if $\left(a\left(\sigma_{n}, t_{n}\right), b\left(\sigma_{n}, t_{n}\right)\right) \rightarrow(a, b) \in \widetilde{\mathcal{U}}_{1}$ and $1 \in B\left(\xi_{\sigma_{n}, t_{n}}\right)$, then

$$
\left|v_{\sigma_{n}, t_{n}}-1\right|>\varepsilon_{0}
$$

where $v_{\sigma_{n}, t_{n}}$ is the unique non-zero $h_{\sigma_{n}, t_{n}}^{\xi}$-critical point in $\mathbb{D}$.

Proof. Suppose the converse. For simplicity, set $v_{n}=v_{\sigma_{n}, t_{n}}$ and $G_{n}=$ $G_{\sigma_{n}, t_{n}}$ for $G_{\sigma_{n}, t_{n}}$ from (28). Passing to a subsequence we can assume $h_{\sigma_{n}, t_{n}}^{\xi}$ $\rightarrow h$, where $h$ is a Blaschke product with a critical point at 0 and a parabolic fixed point at 1 of multiplier 1 . Since $v_{n} \rightarrow 1$ by assumption, we have $\operatorname{deg} h=2$. This easily implies $h=h_{1}$ for $h_{1}$ from (26). Let

$$
\gamma_{n}^{\prime}=\left(\varphi_{\sigma_{n}, t_{n}}^{\xi}\right)^{-1}\left(H_{\sigma_{n}, t_{n}}\left(\varphi_{\sigma_{n}}^{u}\left(\gamma_{\sigma_{n}}^{\prime u}\right)\right)\right), \quad \gamma_{n}^{\prime \prime}=\left(\varphi_{\sigma_{n}, t_{n}}^{\xi}\right)^{-1}\left(H_{\sigma_{n}, t_{n}}\left(\varphi_{\sigma_{n}}^{u}\left(\gamma_{\sigma_{n}}^{\prime \prime u}\right)\right)\right)
$$


for the curves $\gamma_{\sigma_{n}}^{\prime u}, \gamma_{\sigma_{n}}^{\prime \prime}$ from (24) and let

$$
\gamma_{n}=h_{\sigma_{n}, t_{n}}^{\xi}\left(\gamma_{n}^{\prime \prime}\right)
$$

Then $\gamma_{n}^{\prime \prime}$ begins at $G_{n}(-\widehat{r})$ and $\gamma_{n}^{\prime}$ begins at the image under $G_{n}$ of the unique point of $\partial D_{t_{n}} \cap(0,1)$. Moreover,

$$
\gamma_{n}=G_{n}\left(\left[h_{t_{n}}(-\widehat{r}), 1\right) \cap D_{t_{n}}\right) \cup \gamma_{n}^{\prime} .
$$

In particular, $\gamma_{n}$ begins at $G_{n}\left(h_{t_{n}}(-\widehat{r})\right)$, lands at the repelling $h_{\sigma_{n}, t_{n}}^{\xi}$-fixed point 1 , is $h_{\sigma_{n}, t_{n}}^{\xi}$-invariant and contains the attracting $h_{\sigma_{n}, t_{n}}^{\xi}$-fixed point $G_{\sigma_{n}, t_{n}}\left(w_{t_{n}}\right)$ of multiplier $t_{n}$. It is easy to see that we can parameterize $\gamma_{n}$ according to the conditions of the case (b) of Lemma 2.4 with $f_{n}=f_{\sigma_{n}, t_{n}}$, $f=f_{a, b}$ and $\varphi_{\sigma_{n}, t_{n}}^{\xi}, \varphi_{a, b}^{p}$ instead of $\psi_{n}, \psi$.

Recall that $G_{n}$ conjugates holomorphically $h_{t_{n}}$ on $W_{t_{n}}$ to $h_{\sigma_{n}, t_{n}}^{\xi}$. Let

$$
D_{1}=\mathbb{D}_{(1+\widehat{r}) / 2}((1-\widehat{r}) / 2), \quad W_{1}=h_{1}^{-1}\left(D_{1}\right) \cap \mathbb{D} .
$$

Then cl $D_{t_{n}}$ tends to $\mathrm{cl} D_{1}$ and $\mathrm{cl} W_{t_{n}}$ tends to cl $W_{1}$ in the Hausdorff metric. Repeating the proof of Lemma 2.3 we show that $G_{n} \rightarrow G$ almost uniformly on $W_{1}$ for some holomorphic map $G$, in the sense that for any compact set in $W_{1}$ almost all $G_{n}$ are defined on it and tend uniformly to $G$. Moreover, $G$ conjugates $h_{1}$ to $h_{1}$, which easily gives $G=\mathrm{id}$. Let

$$
\gamma^{\prime \prime}=(-1,-\widehat{r}], \quad \gamma=h_{1}\left(\gamma^{\prime \prime}\right)=\left[h_{1}(-\widehat{r}), 1\right) .
$$

Note that $\gamma$ is backward $h_{1}$-invariant and lands at the parabolic $h_{1}$-fixed point 1 . Since $G_{n} \rightarrow$ id and any compact subset of $\left[h_{1}(-\widehat{r}), 1\right)$ is contained in $D_{t_{n}} \cap\left[h_{t_{n}}(-\widehat{r}), 1\right)$ for large $n$, it follows from $(31)$ that $\gamma_{n}(s) \rightarrow \gamma(s)$ almost uniformly for a suitable parameterization of $\gamma$.

To use Lemma 2.4, we need to show

$$
\operatorname{diam} \gamma_{n}([n, \infty)) \rightarrow 0
$$

To prove (32), suppose it is not true. Then in the same way as in the proof of Lemma 2.4 we can find small $\varepsilon_{1}, \varepsilon_{2}>0$ and $s_{j}, n_{j} \rightarrow \infty$ such that $s_{j}>n_{j}$ and

$$
\varepsilon_{1}<\left|\gamma_{n_{j}}\left(s_{j}\right)-1\right|<\varepsilon_{2} \quad \text { and } \operatorname{dist}\left(\gamma_{n_{j}}\left(s_{j}\right),[0,1]\right)<\left|\gamma_{n_{j}}\left(s_{j}\right)-1\right| / 10
$$

for large $j$ (cf. (12)). This together with the almost uniform convergence $G_{n} \rightarrow$ id on $W_{1}$ implies $\gamma_{n_{j}}\left(s_{j}\right) \in G_{n_{j}}\left(W_{t_{n_{j}}}\right)$ and $G_{n_{j}}^{-1}\left(\gamma_{n_{j}}\left(s_{j}\right)\right) \in K \subset W_{1}$ for some compact set $K$ independent of $j$. Hence,

$$
G_{n_{j}}^{-1}\left(\gamma_{n_{j}}\left(s_{j}\right)\right) \in\left[h_{t_{n_{j}}}(-\widehat{r}), r_{1}\right]
$$

for some fixed $r_{1}<1$. This is impossible, because $s_{j}>n_{j}$ and the parameterization of $\gamma_{n_{j}}$ is such that $G_{n_{j}}^{-1}\left(\gamma_{n_{j}}\left(\left(n_{j}, \infty\right)\right) \subset\left(w_{t_{n_{j}}}, 1\right)\right.$ and $w_{t_{n_{j}}} \rightarrow 1$. Hence, (32) holds. 
We have shown that $\gamma_{n}, \gamma$ satisfy all assumptions of the case (b) of Lemma 2.4 with $\varphi_{\sigma_{n}, t_{n}}^{\xi}, \varphi_{a, b}^{p}$ instead of $\psi_{n}, \psi$ (cf. Corollary 4.9). By this lemma, $\operatorname{cl} \gamma_{n}$ tends to $\operatorname{cl} \gamma=\left[h_{1}(-\widehat{r}), 1\right]$ in the Hausdorff metric.

Let $\kappa_{n}$ (resp. $\kappa$ ) be the upper half of the circle, which is symmetric with respect to the real axis and contains $-\widehat{r}, h_{t_{n}}(-\widehat{r})\left(\right.$ resp. $\left.-\widehat{r}, h_{1}(-\widehat{r})\right)$. Note that $\kappa_{n} \subset D_{t_{n}}, \kappa \subset D_{1}$. Define

$$
\Delta_{n}=\gamma_{n} \cup G_{n}\left(\kappa_{n}\right) \cup \gamma_{n}^{\prime \prime}, \quad \Delta=\gamma \cup \kappa \cup \gamma^{\prime \prime}
$$

and let $X_{n}$ be the component of $\mathbb{D} \backslash \Delta_{n}$ which does not contain 0 . Note that $\gamma_{n}^{\prime}, \gamma_{n}^{\prime \prime} \subset \Delta_{n}$ and $G_{n}\left(\partial D_{t_{n}} \cap \mathbb{D}^{+}\right) \subset X_{n}$, so by the definition of $U_{\sigma_{n}, t_{n}}$,

$$
X_{n} \supset\left(\varphi_{\sigma_{n}, t_{n}}^{\xi}\right)^{-1}\left(f_{\sigma_{n}, t_{n}}^{k}\left(U_{\sigma_{n}, t_{n}} \cap B\left(\xi_{\sigma_{n}, t_{n}}\right)\right)\right) .
$$

The definition of $\Delta_{n}$ implies that $\left(h_{\sigma_{n}, t_{n}}^{\xi}\right)^{j}(0),\left(h_{\sigma_{n}, t_{n}}^{\xi}\right)^{j}\left(v_{n}\right) \notin \Delta_{n}$ for $j=0, \ldots, k$, so all branches of $\left(h_{\sigma_{n}, t_{n}}^{\xi}\right)^{-k}$ are defined in a neighbourhood of $\Delta_{n}$. Similarly, all branches of $h_{1}^{-k}$ are defined in a neighbourhood of $\Delta$. It is easy to see that for large $n$ we can extend $G_{n}$ by dynamics to a holomorphic map on $h_{t_{n}}^{-k}\left(D_{t_{n}}\right) \cap \mathbb{D}$, conjugating $h_{t_{n}}$ to $h_{\sigma_{n}, t_{n}}^{\xi}$ and converging almost uniformly to id. Moreover, for $t \in[0,1]$ the map $h_{t}^{k}$ on $\partial\left(h_{t}^{-k}\left(D_{t}\right) \cap \mathbb{D}\right)$ is conjugate to $z \mapsto z^{2^{k}}$ on $\partial \mathbb{D}$ and the conjugation depends continuously on $t \in[0,1]$. These facts together with the definition of $U_{\sigma_{n}, t_{n}}$ easily imply that there exist a branch $\nu_{n}$ of $\left(h_{\sigma_{n}, t_{n}}^{\xi}\right)^{-k}$ defined in a neighbourhood of $\Delta_{n}$ and a branch $\nu$ of $h_{1}^{-k}$ defined in a neighbourhood of $\Delta$, such that if $Y_{n}$ is the component of $\mathbb{D} \backslash \nu_{n}\left(\Delta_{n}\right)$ which does not contain 0 and $Y$ is the component of $\mathbb{D} \backslash \nu(\Delta)$ which does not contain 0 , then

$$
Y_{n} \supset\left(\varphi_{\sigma_{n}, t_{n}}^{\xi}\right)^{-1}\left(U_{\sigma_{n}, t_{n}} \cap B\left(\xi_{\sigma_{n}, t_{n}}\right)\right) \ni v_{n}
$$

$1 \notin \operatorname{cl} Y$ and $\nu_{n}\left(G_{n}\left(\kappa_{n}\right)\right)$ tends to $\nu(\kappa)$ in the Hausdorff metric. Since $h_{\sigma_{n}, t_{n}}^{\xi} \rightarrow h_{1}$ almost uniformly on $\mathrm{cl} \mathbb{D} \backslash\{1\}$ and $\operatorname{cl} \gamma_{n}$ tends to $\mathrm{cl} \gamma$ in the Hausdorff metric, this easily implies that $\partial Y_{n}$ tends to $\partial Y$ in the Hausdorff metric. As $1 \notin \operatorname{cl} Y$, this gives $\operatorname{dist}\left(\operatorname{cl} Y_{n}, 1\right)>c>0$, so $\left|v_{n}-1\right|>c$, which contradicts $v_{n} \rightarrow 1$.

Let $(a, b) \in \widetilde{\mathcal{U}}_{1}$ and take $\sigma_{n} \in \mathcal{U}^{\prime}, t_{n} \rightarrow 1^{-}$such that $\left(a\left(\sigma_{n}, t_{n}\right), b\left(\sigma_{n}, t_{n}\right)\right)$ $\rightarrow(a, b)$. By Proposition 4.14 and Lemma $2.3, f_{a, b}$ has a parabolic fixed point $p_{a, b}$ of multiplier 1 with the unique invariant simply connected parabolic basin $B\left(p_{a, b}\right)$ such that $u \in B\left(p_{a, b}\right), p_{\sigma_{n}, t_{n}} \rightarrow p_{a, b}$ and $\left(B\left(\xi_{\sigma_{n}, t_{n}}\right), u\right)$ tends to $\left(B\left(p_{a, b}\right), u\right)$ in the Carathéodory topology. Similarly, for $\zeta \in\{0, \infty\}$ the immediate basin $B(\zeta)$ for $f_{a, b}$ is simply connected and $(B(\zeta), \zeta)$ for the map $f_{\sigma_{n}, t_{n}}$ tends to $(B(\zeta), \zeta)$ for the map $f_{a, b}$ in the Carathéodory topology.

Note that $f_{a, b}$ on $B\left(p_{a, b}\right)$ is conjugate by a Riemann map to a quadratic or cubic Blaschke product $h_{a, b}^{p}$ with a (unique) fixed point of multiplier 1 
in $\partial \mathbb{D}$. Let

$$
\varphi_{a, b}^{p}: \mathbb{D} \rightarrow B\left(p_{a, b}\right)
$$

be the unique Riemann mapping such that $\varphi_{a, b}^{p}(0)=u$ and the $h_{a, b}^{p}$-fixed point of multiplier 1 is equal to 1 . It is easy to check that $\varphi_{a, b}^{p}$ depends continuously on $(a, b) \in \widetilde{\mathcal{U}}_{1}$ in the uniform convergence topology.

Recall that $f_{\sigma_{n}, t_{n}}$ on $B(\infty)$ is conjugate by $\varphi_{\sigma_{n}, t_{n}}^{\infty}$ to $h_{\sigma_{n}}^{\infty}$ for $h_{\sigma_{n}}^{\infty}$ from (17). By Corollary 4.9 , it is easy to see that for $(a, b) \in \widetilde{\mathcal{U}}_{1}$ we can choose a Riemann map

$$
\varphi_{a, b}^{\infty}: \mathbb{D} \rightarrow B(\infty)
$$

such that $\varphi_{a, b}^{\infty}(0)=\infty, \varphi_{\sigma_{n}, t_{n}}^{\infty} \rightarrow \varphi_{a, b}^{\infty}$ almost uniformly on $\mathbb{D}$ and $\varphi_{a, b}^{\infty}$ depends continuously on $(a, b) \in \widetilde{\mathcal{U}}_{1}$.

By Lemma 4.17 we easily obtain

COROLlary 4.18. If $(a, b) \in \widetilde{\mathcal{U}}_{1}$ and $\sigma_{n} \in \mathcal{U}^{\prime}, t_{n} \rightarrow 1^{-}$are such that $\left(a\left(\sigma_{n}, t_{n}\right), b\left(\sigma_{n}, t_{n}\right)\right) \rightarrow(a, b)$, then for $\zeta \in\{\xi, \infty\}$ Lemmas 2.3 and 2.4 hold for $f_{n}=f_{\sigma_{n}, t_{n}}, f=f_{a, b}$ and $\varphi_{\sigma_{n}, t_{n}}^{\zeta}, \varphi_{a, b}^{\zeta}$ instead of $\psi_{n}, \psi$. In particular, $h_{\sigma_{n}, t_{n}}^{\xi} \rightarrow h_{a, b}^{p}$ and there exists $\sigma_{a, b} \in \mathrm{cl}^{\prime}$ such that $h_{\sigma_{n}}^{\infty} \rightarrow h_{\sigma_{a, b}}^{\infty}$ and $\varphi_{a, b}^{\infty}$ conjugates $\left.f_{a, b}\right|_{B(\infty)}$ to $h_{\sigma_{a, b}}^{\infty}$. Moreover, the convergence of $h_{\sigma_{n}, t_{n}}^{\xi}$ and $h_{\sigma_{n}}^{\infty}$ is almost uniform in the spherical metric on $\widehat{\mathbb{C}} \backslash\left\{z_{0}\right\}$, where $z_{0} \in \partial \mathbb{D} \backslash\{1\}$.

REMARK. The parameter $\sigma_{a, b}$ is not uniquely defined, but $h_{\sigma_{a, b}}^{\infty}$ is unique.

Note that this corollary implies that for $(a, b) \in M_{1}$ we have $\operatorname{deg} h_{a, b}^{p}=$ $\operatorname{deg} h_{\sigma_{a, b}}=2$, which clearly gives $h_{a, b}^{p}=h_{1}$ for $h_{1}$ from (26) and $h_{\sigma_{a, b}}^{\infty}(z)=$ $z^{2}$ in this case.

Now we show that

$$
1 \notin f_{a, b}^{-(k+1)}\left(p_{a, b}\right) \quad \text { for every }(a, b) \in \tilde{\mathcal{U}}_{1} .
$$

By the compactness of $M_{1}$, if we take sufficiently small $\varepsilon_{1}$ in the definition of $\widetilde{\mathcal{U}}_{1}$, then it is sufficient to check (33) for $(a, b) \in M_{1}$. Suppose that (33) does not hold. Then $f_{a, b}^{2 k+1}(1)=p_{a, b}$. Take $\sigma_{n}, t_{n}$ as above. Note that if we take $\mathcal{U}^{\prime}$ sufficiently close to $M_{N}$, then $N_{\sigma}^{2 k+1}(1) \in N_{\sigma}\left(U_{\sigma}^{\prime}\right)$ for $\sigma \in \mathcal{U}^{\prime}$, so $f_{\sigma_{n}, t_{n}}^{2 k+1}(1) \in f_{\sigma_{n}, t_{n}}\left(U_{\sigma_{n}, t_{n}}\right)$. Moreover, since $(a, b) \in M_{1}$, we have $h_{\sigma_{n}, t_{n}}^{\xi} \rightarrow h_{1}$ and $h_{\sigma_{n}}^{\infty}(z) \rightarrow z^{2}$. In the same way as in the proof of Lemma 4.17, using Lemma 2.4 we show that for large $n$,

$$
\partial f_{\sigma_{n}, t_{n}}\left(U_{\sigma_{n}, t_{n}}\right) \subset \mathbb{D}_{\varepsilon}(x) \cup \mathbb{D}_{\varepsilon}(y) \cup \varphi_{\sigma_{n}, t_{n}}^{\xi}\left(\mathbb{D}_{r}\right) \cup \varphi_{\sigma_{n}, t_{n}}^{\infty}\left(\mathbb{D}_{r}\right)
$$

for $x, y \in f_{a, b}^{-k}\left(p_{a, b}\right) \backslash\left\{p_{a, b}\right\}$, some fixed $r<1$ and a small $\varepsilon>0$. This implies $\left|f_{\sigma_{n}, t_{n}}^{2 k+1}(1)-p_{a, b}\right|>$ const $>0$, which contradicts $f_{a, b}^{2 k+1}(1)=p_{a, b}$. In this way we have proved (33). 
Now for $(a, b) \in \widetilde{\mathcal{U}}_{1}$ we define curves $\Gamma_{a, b}^{p}, \Gamma_{a, b}^{\infty}$ in $\mathbb{D}$, similar to the curves $\Gamma_{\sigma}^{u}, \Gamma_{\sigma}^{\infty}$ from (24). To define the curve $\Gamma_{a, b}^{p}$, consider the map $G_{\sigma_{n}, t_{n}}$ from (28). Recall that $G_{\sigma_{n}, t_{n}}$ is a biholomorphic map from $W_{t_{n}}$ into $\mathbb{D}$ conjugating $h_{t_{n}}$ to $h_{\sigma_{n}, t_{n}}^{\xi}$. Let

$$
D_{1}=\mathbb{D}_{(1+\widehat{r}) / 2}((1-\widehat{r}) / 2), \quad W_{1}=h_{1}^{-1}\left(D_{1}\right) \cap \mathbb{D} .
$$

Then $D_{1}$ is the horodisc tangent to $\partial \mathbb{D}$ at 1 such that $-\widehat{r} \in \partial D_{1}$. It is easy to check that

$$
W_{1} \supset\left(\operatorname{cl} D_{1} \backslash\{1\}\right) \cup(-1,1) .
$$

Moreover, $\operatorname{cl} W_{t_{n}}$ tends to $\mathrm{cl} W_{1}$ in the Hausdorff metric. In the same way as in the proof of Lemma 2.3 we show that $G_{\sigma_{n}, t_{n}}$ tends almost uniformly on $W_{1}$ to some holomorphic map $G_{a, b}$ conjugating $h_{1}$ to $h_{a, b}^{p}$, in the sense that for any compact set in $W_{1}$ almost all $G_{\sigma_{n}, t_{n}}$ are defined on it and tend uniformly to $G_{a, b}$. Let

$$
\Gamma_{a, b}^{p}=G_{a, b}\left(\left(\partial D_{1} \cap \mathbb{D}^{+}\right) \cup(-1,-\widehat{r}]\right) .
$$

By definition, $\Gamma_{a, b}^{p}$ is a simple arc in $\mathbb{D}$ connecting 1 to -1 (see Fig. 10).

Note that if $(a, b) \in M_{1}$, then $G_{a, b}$ conjugates $h_{1}$ to $h_{1}$, which easily gives $G_{a, b}=$ id. Hence, $\Gamma_{a, b}^{p}=\left(\partial D_{1} \cap \mathbb{D}^{+}\right) \cup(-1,-\widehat{r}]$ in this case.

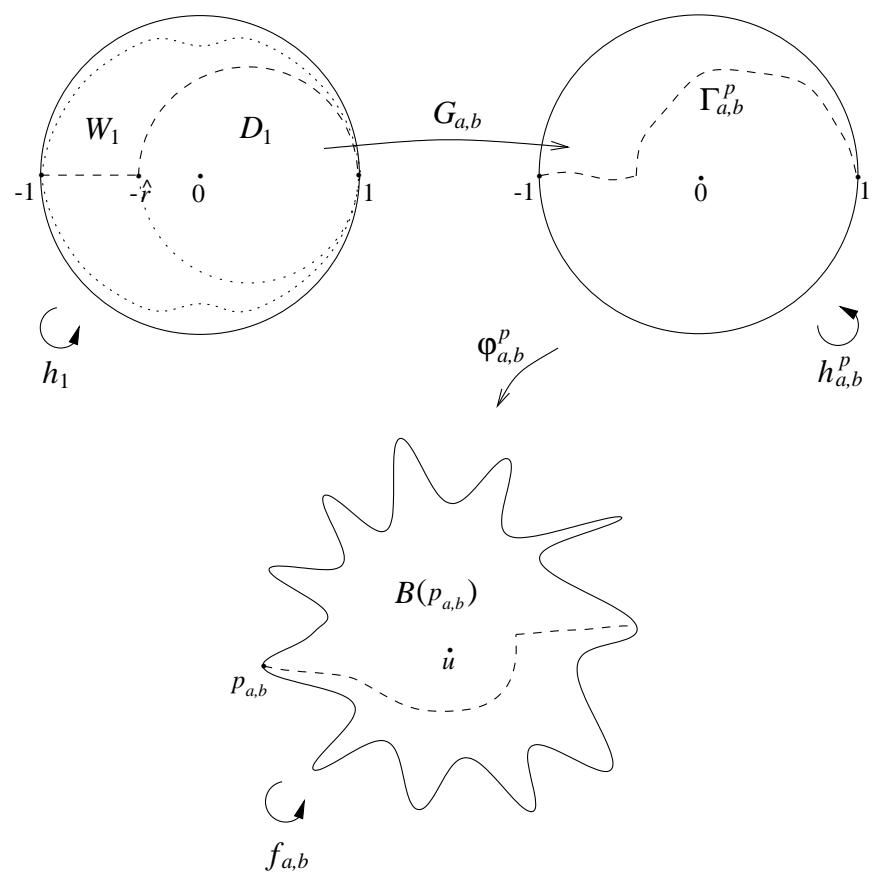

Fig. 10. The sets $D_{1}, W_{1}$ and the curve $\Gamma_{a, b}^{p}$ 
Recall that by Corollary $4.18, f_{a, b}$ on $B(\infty)$ is conjugate by $\varphi_{a, b}^{\infty}$ to $h_{\sigma_{a, b}}^{\infty}$ for some $\sigma_{a, b} \in \operatorname{cl} \mathcal{U}^{\prime}$. Let

$$
\Gamma_{a, b}^{\infty}=\Gamma_{\sigma_{a, b}}^{\infty}
$$

for $\Gamma_{\sigma_{a, b}}^{\infty}$ from (24). It is easy to see that $\Gamma_{a, b}^{p}$ and $\Gamma_{a, b}^{\infty}$ do not depend on the choice of the sequences $\sigma_{n}, t_{n}$.

Proposition 4.19. For every $(a, b) \in \widetilde{\mathcal{U}}_{1}$ and $\sigma_{n} \in \mathcal{U}^{\prime}, t_{n} \rightarrow 1^{-}$such that $\left(a\left(\sigma_{n}, t_{n}\right), b\left(\sigma_{n}, t_{n}\right)\right) \rightarrow(a, b)$,

$$
\operatorname{cl}\left(\varphi_{a, b}^{p}\left(\Gamma_{a, b}^{p}\right) \cup \varphi_{a, b}^{\infty}\left(\Gamma_{a, b}^{\infty}\right)\right)
$$

is a Jordan curve and $\partial f_{\sigma_{n}, t_{n}}^{k}\left(U_{\sigma_{n}, t_{n}}\right)$ tends to it in the Hausdorff metric.

The proof is split into two lemmas. The first proves that the curves $\varphi_{a, b}^{\infty}\left(\gamma_{\sigma_{a, b}}^{\prime \prime \infty}\right)$ and $\varphi_{a, b}^{p}\left(G_{a, b}((-1,-\widehat{r}])\right)$ have a common landing point and the second shows the same for $\varphi_{a, b}^{\infty}\left(\gamma_{\sigma_{a, b}}^{\prime \infty}\right)$ and $\varphi_{a, b}^{p}\left(G_{a, b}\left(\partial D_{1} \cap \mathbb{D}^{+}\right)\right.$) (where $\gamma_{\sigma_{a, b},}^{\prime \infty}, \gamma_{\sigma_{a, b}}^{\prime \prime \infty}$ are the curves from $\left.(24)\right)$.

Lemma 4.20. For $(a, b), \sigma_{n}, t_{n}$ as in Proposition 4.19, $\varphi_{a, b}^{\infty}\left(\gamma_{\sigma_{a, b}}^{\prime \prime}\right)$ and $\varphi_{a, b}^{p}\left(G_{a, b}((-1,-\widehat{r}])\right)$ land at the same point and $\operatorname{cl}\left(H_{\sigma_{n}, t_{n}}\left(\varphi_{\sigma_{n}}^{\infty}\left(\gamma_{\sigma_{n}}^{\prime \prime \infty}\right)\right) \cup\right.$ $\left.H_{\sigma_{n}, t_{n}}\left(\varphi_{\sigma_{n}}^{u}\left(\gamma_{\sigma_{n}}^{\prime \prime \prime}\right)\right)\right)$ tends to $\operatorname{cl}\left(\varphi_{a, b}^{\infty}\left(\gamma_{\sigma_{a, b}}^{\prime \prime}\right) \cup \varphi_{a, b}^{p}\left(G_{a, b}((-1,-\widehat{r}])\right)\right)$ in the Hausdorff metric. Moreover, $\mathrm{cl} H_{\sigma_{n}, t_{n}}\left(\varphi_{\sigma_{n}}^{\infty}\left(\gamma_{\sigma_{n}}^{\prime \infty}\right)\right)$ tends to $\mathrm{cl} \varphi_{a, b}^{\infty}\left(\gamma_{\sigma_{a, b}}^{\prime \infty}\right)$ in the Hausdorff metric.

Proof. Define $\gamma_{n}^{\prime}, \gamma_{n}^{\prime \prime}$ and $\gamma_{n}$ as in the proof of Lemma 4.17 and let

$$
\gamma^{\prime \prime}=G_{a, b}((-1,-\widehat{r}]), \quad \gamma=h_{a, b}^{p}\left(\gamma^{\prime \prime}\right)=G_{a, b}\left(\left[h_{1}(-\widehat{r}), 1\right)\right) .
$$

In the same way as in the proof of Lemma 4.17 we show that we can parameterize $\gamma_{n}, \gamma$ according to the conditions of the case (b) of Lemma 2.4 so that $\gamma_{n}(s) \rightarrow \gamma(s)$ almost uniformly.

To use Lemma 2.4, we need to show (32) in this case. Suppose it does not hold. In the same way as in the proof of Lemma 2.4 we find $s_{n}>n$ such that (after passing to a subsequence) we have $\gamma_{n}\left(s_{n}\right) \in K$ for a fixed compact set $K \subset \mathbb{D}$. Since $\mathbb{D}$ is the invariant attracting basin for the parabolic $h_{a, b}^{p}$-fixed point 1 of multiplier 1 and $h_{\sigma_{n}, t_{n}}^{\xi} \rightarrow h_{a, b}^{p}$ almost uniformly on $\mathbb{D}$, there exist arbitrarily large $j, m>0$ such that

$$
\left|\left(h_{\sigma_{n}, t_{n}}^{\xi}\right)^{j}\left(\gamma_{n}\left(s_{n}\right)\right)-\left(h_{\sigma_{n}, t_{n}}^{\xi}\right)^{m}(0)\right|<\left|\left(h_{\sigma_{n}, t_{n}}^{\xi}\right)^{m}(0)-\left(h_{\sigma_{n}, t_{n}}^{\xi}\right)^{m+1}(0)\right|
$$

for sufficiently large $n$. By the definition of $G_{\sigma_{n}, t_{n}}$, for every $l \geq 0$ we have

$$
\left(h_{\sigma_{n}, t_{n}}^{\xi}\right)^{l}(0)=G_{\sigma_{n}, t_{n}}\left(h_{t_{n}}^{l}(0)\right) .
$$

Moreover, for almost all $n$ the map $G_{\sigma_{n}, t_{n}}$ is defined and has universally bounded distortion on

$$
D^{(n)}=\mathbb{D}_{\left|h_{t_{n}}^{m}(0)-w_{t_{n}}\right| / 2}\left(h_{t_{n}}^{m}(0)\right)
$$


and $\left|h_{t_{n}}^{m}(0)-h_{t_{n}}^{m+1}(0)\right| /\left|h_{t_{n}}^{m}(0)-w_{t_{n}}\right|$ is arbitrarily small provided $m$ is chosen sufficiently large. By this and $(35),\left(h_{\sigma_{n}, t_{n}}^{\xi}\right)^{j}\left(\gamma_{n}\left(s_{n}\right)\right) \in G_{\sigma_{n}, t_{n}}\left(D^{(n)}\right)$ for large $n$. But since $s_{n}>n$, we have $\left(h_{\sigma_{n}, t_{n}}^{\xi}\right)^{j}\left(\gamma_{n}\left(s_{n}\right)\right)=\gamma_{n}\left(s_{n}^{\prime}\right)$ for some $s_{n}^{\prime}$ $>n$. This is a contradiction, because the definition of $\gamma_{n}$ implies $G_{\sigma_{n}, t_{n}}\left(D^{(n)}\right)$ $\cap \gamma_{n}((n, \infty))=\emptyset$. This shows (32) in our case.

We have checked that $\gamma_{n}, \gamma$ satisfy all assumptions of the case (b) of Lemma 2.4 with $h_{n}=h_{\sigma_{n}, t_{n}}^{\xi}, h=h_{a, b}^{p}$ and $\varphi_{\sigma_{n}, t_{n}}^{\xi}, \varphi_{a, b}^{p}$ instead of $\psi_{n}, \psi$. Note also that the curves $\gamma_{\sigma_{n}}^{\infty}, \gamma_{\sigma_{a, b}}^{\infty}$ with suitable parameterizations fulfill the conditions of the case (a) of that lemma with $h_{n}=h_{\sigma_{n}}^{\infty}, h=h_{\sigma_{a, b}}^{\infty}$ and $\varphi_{\sigma_{n}, t_{n}}^{\infty}, \varphi_{a, b}^{\infty}$ instead of $\psi_{n}, \psi$. By Corollary 4.18 and Lemma 2.4, both curves $\varphi_{a, b}^{p}(\gamma)$ and $\varphi_{a, b}^{\infty}\left(\gamma_{\sigma_{a, b}}^{\infty}\right)$ land at $p_{a, b}$ and for every $\varepsilon>0$ we can find $s_{0}$ such that

$$
\varphi_{\sigma_{n}, t_{n}}^{\xi}\left(\gamma_{n}\left(\left[s_{0}, \infty\right)\right)\right), \varphi_{\sigma_{n}, t_{n}}^{\infty}\left(\gamma_{\sigma_{n}}^{\infty}\left(\left[s_{0}, \infty\right)\right)\right) \subset \mathbb{D}_{\varepsilon}\left(p_{a, b}\right)
$$

for large $n$. Note that the curves $\varphi_{\sigma_{n}, t_{n}}^{\xi}\left(\gamma_{n}\right)$ and $\varphi_{\sigma_{n}}^{\infty}\left(\gamma_{\sigma_{n}}^{\infty}\right)$ are contained in $\partial f_{\sigma_{n}, t_{n}}\left(U_{\sigma_{n}, t_{n}}\right)$ and land at $p_{\sigma_{n}, t_{n}}$.

Since $\varphi_{a, b}^{p}\left(G_{a, b}((-1,-\widehat{r}])\right)$ is a preimage under $f_{a, b}^{-1}$ of $\varphi_{a, b}^{p}(\gamma)$, it has a well-defined landing point $x \in f_{a, b}^{-1}\left(p_{a, b}\right) \backslash\left\{p_{a, b}\right\}$. Similarly, $\varphi_{a, b}^{\infty}\left(\gamma_{\sigma_{a, b}}^{\prime \prime}\right)$ lands at a point $y \in f_{a, b}^{-1}\left(p_{a, b}\right) \backslash\left\{p_{a, b}\right\}$. By (33), $f_{a, b}$ maps biholomorphically a small neighbourhood of $x$ and $y$ onto a neighbourhood of $p_{a, b}$. Hence, there exists $\varepsilon^{\prime}>0$, which is arbitrarily small if $\varepsilon$ is small enough, such that $f_{\sigma_{n}, t_{n}}\left(\mathbb{D}_{\varepsilon^{\prime}}(x)\right), f_{\sigma_{n}, t_{n}}\left(\mathbb{D}_{\varepsilon^{\prime}}(y)\right) \supset \mathbb{D}_{\varepsilon}\left(p_{a, b}\right)$ for large $n$. Moreover, by the almost uniform convergence of $\varphi_{\sigma_{n}, t_{n}}^{\xi}, \varphi_{\sigma_{n}, t_{n}}^{\infty}$, the disc $\mathbb{D}_{\varepsilon^{\prime}}(x)$ contains points from $H_{\sigma_{n}, t_{n}}\left(\varphi_{\sigma_{n}}^{u}\left(\gamma_{\sigma_{n}}^{\prime \prime \prime}\right)\right)$ and $\mathbb{D}_{\varepsilon^{\prime}}(y)$ contains points from $\varphi_{\sigma_{n}, t_{n}}^{\infty}\left(\gamma_{\sigma_{n}}^{\prime \prime \infty}\right)$ for large $n$. Therefore, $H_{\sigma_{n}, t_{n}}\left(\varphi_{\sigma_{n}}^{u}\left(\gamma_{\sigma_{n}}^{\prime \prime}\right)\right)$ lands at some point in $\mathbb{D}_{\varepsilon^{\prime}}(x)$ and $\varphi_{\sigma_{n}, t_{n}}^{\infty}\left(\gamma_{\sigma_{n}}^{\prime \prime \prime}\right)$ lands at some point in $\mathbb{D}_{\varepsilon^{\prime}}(y)$. But by the definition of $U_{\sigma, t}$, these curves are contained in $\partial f_{\sigma_{n}, t_{n}}^{k}\left(U_{\sigma_{n}, t_{n}}\right)$ and land at the same point. This implies $x=y$, because otherwise we have a contradiction for $\varepsilon^{\prime}<|x-y| / 2$. In this way we have shown that the curves $\varphi_{a, b}^{p}\left(G_{a, b}((-1,-\widehat{r}])\right)$ and $\varphi_{a, b}^{\infty}\left(\gamma_{\sigma_{a, b}}^{\prime \prime}\right)$ land at the same point. Moreover, by Lemma 2.4, $\operatorname{cl} \varphi_{\sigma_{n}, t_{n}}^{\xi}\left(\gamma_{n}\right)$ tends to $\operatorname{cl} \varphi_{a, b}^{p}(\gamma)$ in the Hausdorff metric, so $\operatorname{cl} H_{\sigma_{n}, t_{n}}\left(\varphi_{\sigma_{n}}^{u}\left(\gamma_{\sigma_{n}}^{\prime \prime u}\right)\right)=\operatorname{cl} \varphi_{\sigma_{n}, t_{n}}^{\xi}\left(\gamma_{\sigma_{n}}^{\prime \prime u}\right)$ tends to $\operatorname{cl} \varphi_{a, b}^{p}\left(G_{a, b}((-1,-\widehat{r}])\right)=\operatorname{cl} \varphi_{a, b}^{p}\left(\gamma^{\prime \prime}\right)$. Similarly, $\operatorname{cl} \varphi_{\sigma_{n}, t_{n}}^{\infty}\left(\gamma_{\sigma_{n}}^{\infty}\right)$ tends to $\operatorname{cl} \varphi_{a, b}^{\infty}\left(\gamma_{\sigma_{a, b}}^{\infty}\right)$, which implies that $\operatorname{cl} H_{\sigma_{n}, t_{n}}\left(\varphi_{\sigma_{n}}^{\infty}\left(\gamma_{\sigma_{n}}^{\prime \infty}\right)\right)=\operatorname{cl} \varphi_{\sigma_{n}, t_{n}}^{\infty}\left(\gamma_{\sigma_{n}}^{\prime \infty}\right)$ tends to $\left.\operatorname{cl} \varphi_{a, b}^{\infty}\left(\gamma_{\sigma_{a, b}}^{\prime \infty}\right)\right)$ and $\operatorname{cl} H_{\sigma_{n}, t_{n}}\left(\varphi_{\sigma_{n}}^{\infty}\left(\gamma_{\sigma_{n}}^{\prime \prime \infty}\right)\right)=\operatorname{cl} \varphi_{\sigma_{n}, t_{n}}^{\infty}\left(\gamma_{\sigma_{n}}^{\prime \prime \infty}\right)$ tends to $\operatorname{cl} \varphi_{a, b}^{\infty}\left(\gamma_{\sigma_{a, b}}^{\prime \prime \infty}\right)$. This ends the proof of the lemma.

Note that by Lemma $2.4, \varphi_{a, b}^{\infty}\left(\gamma_{\sigma_{a, b}}^{\prime \infty}\right)$ lands at $p_{a, b}$. Now we show that $\varphi_{a, b}^{p}\left(G_{a, b}\left(\partial D_{1} \cap \mathbb{D}^{+}\right)\right)$lands at $p_{a, b}$.

Lemma 4.21. For $(a, b), \sigma_{n}, t_{n}$ as in Proposition $4.19, G_{a, b}\left(\partial D_{1} \cap \mathbb{D}^{+}\right)$ lands at 1 and $\varphi_{a, b}^{p}\left(G_{a, b}\left(\partial D_{1} \cap \mathbb{D}^{+}\right)\right)$lands at $p_{a, b}$. Moreover, if $z_{n} \in \partial D_{t_{n}}$ 
and $z_{n} \rightarrow 1$, then $G_{\sigma_{n}, t_{n}}\left(z_{n}\right) \rightarrow 1$ and $\varphi_{\sigma_{n}, t_{n}}^{\xi}\left(G_{\sigma_{n}, t_{n}}\left(z_{n}\right)\right) \rightarrow p_{a, b}$. Furthermore, cl $H_{\sigma_{n}, t_{n}}\left(\varphi_{\sigma_{n}}^{u}\left(\Gamma_{\sigma_{n}}^{u} \backslash \gamma_{\sigma_{n}}^{\prime \prime u}\right)\right)$ tends to $\operatorname{cl} \varphi_{a, b}^{p}\left(G_{a, b}\left(\partial D_{1} \cap \mathbb{D}^{+}\right)\right)$in the Hausdorff metric.

Proof. Let

$$
J_{\sigma_{n}}^{ \pm}=\bigcup_{j=0}^{\infty}\left(\nu_{\sigma_{n}}^{\prime u}\right)^{j}\left(\left(\Phi_{\sigma_{n}}^{u}\right)^{-1}\left(\left[\sqrt{\widehat{r}} e^{ \pm i \theta_{0}}, \widehat{r} e^{ \pm 2 i \theta_{0}}\right]\right)\right)
$$

for the inverse branch $\nu_{\sigma_{n}}^{\prime u}$ of $\left(h_{\sigma_{n}}^{u}\right)^{-1}$ used in the definition of the curve $\gamma_{\sigma_{n}}^{\prime u}$ and a small fixed $\theta_{0}>0$ (by $[x, y]$ we mean here the straight line segment between $x$ and $y$ ). Then $J_{\sigma_{n}}^{ \pm}$are two backward $h_{\sigma_{n}}^{u}$-invariant curves in $\mathbb{D}$ landing at 1 and

$$
\Phi_{\sigma_{n}}^{-1}\left(\left\{\widehat{r} e^{i \theta}: \theta \in\left[2 \theta_{0}, 2 \pi-2 \theta_{0}\right]\right\}\right) \cup J_{\sigma_{n}}^{+} \cup J_{\sigma_{n}}^{-} \cup\{1\}
$$

is a Jordan curve. Define $P_{\sigma_{n}}$ to be the open domain in $\mathbb{D}$ such that $0 \in P_{\sigma_{n}}$ and $\partial P_{\sigma_{n}}$ is equal to this curve. It is easy to check that $h_{\sigma_{n}}^{u}\left(\operatorname{cl} P_{\sigma_{n}}\right) \subset \operatorname{cl} P_{\sigma_{n}}$. Let

$$
\begin{aligned}
& P_{n}=\left(\varphi_{\sigma_{n}, t_{n}}^{\xi}\right)^{-1}\left(H_{\sigma_{n}, t_{n}}\left(\varphi_{\sigma_{n}}^{u}\left(P_{\sigma_{n}}\right)\right)\right), \\
& J_{n}^{ \pm}=\left(\varphi_{\sigma_{n}, t_{n}}^{\xi}\right)^{-1}\left(H_{\sigma_{n}, t_{n}}\left(\varphi_{\sigma_{n}}^{u}\left(J_{\sigma_{n}}^{ \pm}\right)\right) .\right.
\end{aligned}
$$

Then $P_{n}$ are topological discs in $\mathbb{D}, \partial P_{n} \cap \partial \mathbb{D}=\{1\}$ and $h_{\sigma_{n}, t_{n}}^{\xi}\left(\operatorname{cl} P_{n}\right) \subset \operatorname{cl} P_{n}$ (see Fig. 11).
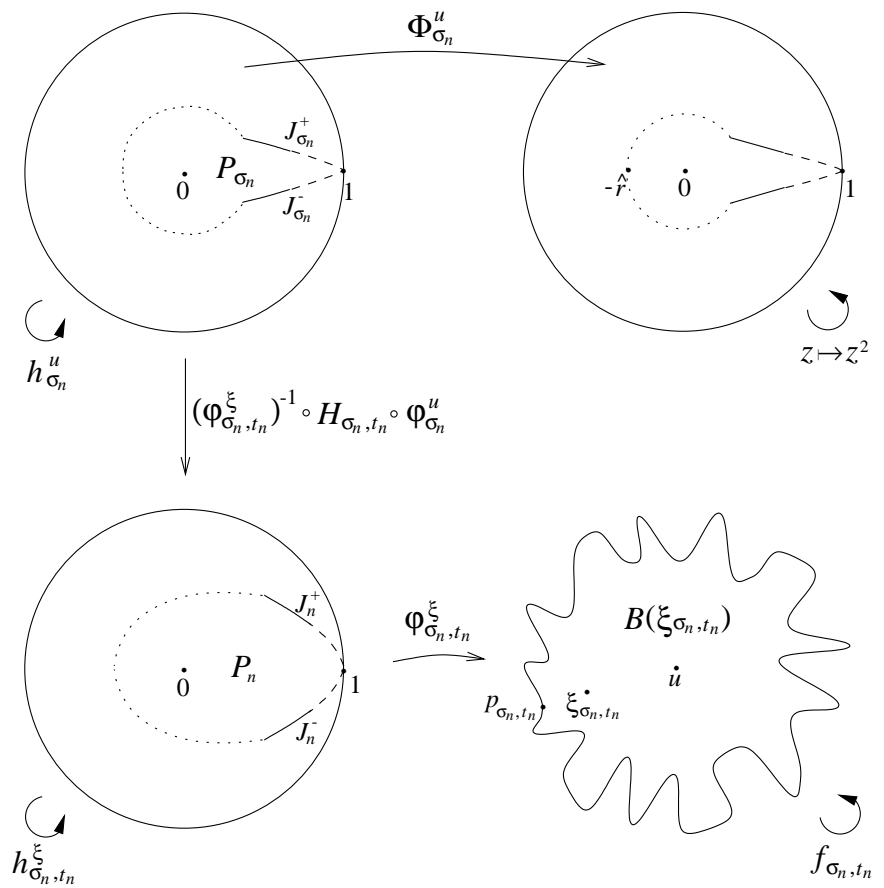

Fig. 11. The curves $J_{n}^{ \pm}$and the set $P_{n}$ 
Moreover, by the definitions of $F_{\sigma, t}$ and $G_{\sigma, t}$, there exists a sequence $y_{n}>0$ such that $y_{n} \rightarrow y_{0}>0$ and

$$
\begin{aligned}
\partial P_{n} & =G_{\sigma_{n}, t_{n}}\left(\partial D_{t_{n}} \backslash \mathbb{D}_{y_{n}}(1)\right) \cup J_{n}^{+} \cup J_{n}^{-} \cup\{1\}, \\
P_{n} & \supset G_{\sigma_{n}, t_{n}}\left(\partial D_{t_{n}} \cap \mathbb{D}_{y_{n}}(1)\right) .
\end{aligned}
$$

Parameterize the curves $J_{n}^{ \pm}$according to the conditions of the case (a) of Lemma 2.4. It is easy to check that $J_{n}^{ \pm}$tend to some backward $h_{1}$-invariant curves $J^{ \pm}$in $\mathbb{D}$, which begin at two points of $G_{a, b}\left(\partial D_{1} \cap \mathbb{D}_{y_{0}}(1)\right)$. By Lemma $2.4, J^{ \pm}$lands at 1 , so

$$
G_{a, b}\left(\partial D_{1} \backslash \mathbb{D}_{y_{0}}(1)\right) \cup J^{+} \cup J^{-} \cup\{1\}
$$

is a Jordan curve. Let $P$ be the topological disc in $\mathbb{D}$ such that $0 \in P$ and $\partial P$ is equal to this curve. Then $\partial P \cap \partial \mathbb{D}=\{1\}, G_{a, b}\left(\partial D_{1} \cap\left(\mathbb{D}_{y_{0}}(1) \backslash\{1\}\right)\right) \subset P$ and $h_{a, b}^{p}(\operatorname{cl} P) \subset \operatorname{cl} P$. These facts easily imply that $G_{a, b}\left(\partial D_{1} \cap \mathbb{D}^{+}\right)$lands at 1 .

Let $z_{n} \in \partial D_{t_{n}}, z_{n} \rightarrow 1$ and suppose $G_{\sigma_{n}, t_{n}}\left(z_{n}\right) \nrightarrow \rightarrow 1$. Passing to a subsequence assume $\left|G_{\sigma_{n}, t_{n}}\left(z_{n}\right)-1\right|>\varepsilon$ for a fixed $\varepsilon>0$. By (37), we have $G_{\sigma_{n}, t_{n}}\left(z_{n}\right) \in P_{n}$ for large $n$. Using Lemma 2.4 for the curves $J_{n}^{ \pm}$we get $G_{\sigma_{n}, t_{n}}\left(z_{n}\right) \in K$ for a fixed compact set $K \in \mathbb{D}$. Hence, (35) holds for $G_{\sigma_{n}, t_{n}}\left(z_{n}\right)$ instead of $\gamma_{n}\left(s_{n}\right)$. Repeating the arguments from the proof of Lemma 4.20 we show that for large $n$,

$$
\left(h_{\sigma_{n}, t_{n}}^{\xi}\right)^{j}\left(G_{\sigma_{n}, t_{n}}\left(z_{n}\right)\right) \in G_{\sigma_{n}, t_{n}}\left(D^{(n)}\right)
$$

for $D^{(n)}$ from (36), which gives $h_{t_{n}}^{j}\left(z_{n}\right) \in D^{(n)}$. But $h_{t_{n}} \rightarrow h_{1}$ uniformly on cl $\mathbb{D}$, so $h_{t_{n}}^{j}\left(z_{n}\right) \rightarrow 1$. This is a contradiction, since by the definition of $D^{(n)}$, we have $\operatorname{dist}\left(\operatorname{cl} D^{(n)}, 1\right)>$ const $>0$. Hence, $G_{\sigma_{n}, t_{n}}\left(z_{n}\right) \rightarrow 1$.

By Lemma 2.4, the curves $\varphi_{a, b}^{p}\left(J^{ \pm}\right)$land at $p_{a, b}$, so $\varphi_{a, b}^{p}(\partial P \backslash\{1\}) \cup\left\{p_{a, b}\right\}$ is a Jordan curve. Let $S \subset \widehat{\mathbb{C}}$ be the component of the complement of this Jordan curve containing $u=\varphi_{a, b}^{p}(0)$. Then $S \supset \varphi_{a, b}^{p}(P)$ and $\partial S \subset$ $B\left(p_{a, b}\right) \cup\left\{p_{a, b}\right\}$. Moreover, we have $\infty \notin S$ by Lemma 4.20. Now we show that the unique point from $f_{a, b}^{-1}(\infty) \backslash\{\infty\}$ is not in $S$. This is obvious if $\left.\operatorname{deg} f_{a, b}\right|_{B(\infty)}=3$. If $\left.\operatorname{deg} f_{a, b}\right|_{B(\infty)}=2$, then there exists a unique component $\widetilde{B}(\infty)$ of $f_{a, b}^{-1}(B(\infty)) \backslash B(\infty)$. In the same way as for Lemma 4.20 we show that some preimage under $f_{a, b}^{-(k-1)}$ of the curve $\varphi_{a, b}^{p}\left(G_{a, b}((-1,-\widehat{r}])\right)$ is contained in $B\left(p_{a, b}\right)$ and lands at the same point as some preimage of the curve $\varphi_{a, b}^{\infty}\left(\gamma_{\sigma_{a, b}}^{\prime \prime}\right)$ contained in $\widetilde{B}(\infty)$. This gives $\widetilde{B}(\infty) \cap S=\emptyset$. Hence, we have $f_{a, b}^{-1}(\infty) \cap \operatorname{cl} S=\emptyset$. Moreover, $f_{a, b}(\partial S) \subset \operatorname{cl} S$ by the definition of $S$. Hence, by the maximum principle, $f_{a, b}(\operatorname{cl} S) \subset \operatorname{cl} S$. This implies that $S$ does not contain points from the Julia set of $f_{a, b}$, which gives

$$
S=\varphi_{a, b}^{p}(P) \subset B\left(p_{a, b}\right) .
$$

This easily implies that $\varphi_{a, b}^{p}\left(G_{a, b}\left(\partial D_{1} \cap \mathbb{D}^{+}\right)\right)$lands at $p_{a, b}$. 
Suppose $\varphi_{\sigma_{n}, t_{n}}^{\xi}\left(G_{\sigma_{n}, t_{n}}\left(z_{n}\right)\right) \nrightarrow p_{a, b}$. Passing to a subsequence assume $\varphi_{\sigma_{n}, t_{n}}^{\xi}\left(G_{\sigma_{n}, t_{n}}\left(z_{n}\right)\right) \rightarrow x \neq p_{a, b}$. By $(37), \varphi_{\sigma_{n}, t_{n}}^{\xi}\left(G_{\sigma_{n}, t_{n}}\left(z_{n}\right)\right) \in \varphi_{\sigma_{n}, t_{n}}^{\xi}\left(P_{n}\right)$, so by Lemma 2.4 for the curves $J_{n}^{ \pm}$we get $x \in \operatorname{cl} \varphi_{a, b}^{p}(P) \backslash\left\{p_{a, b}\right\} \subset B\left(p_{a, b}\right)$. This leads to a contradiction with $G_{\sigma_{n}, t_{n}}\left(z_{n}\right) \rightarrow 1$.

Note that $\gamma_{\sigma_{n}}^{\prime u} \subset P_{\sigma_{n}} \backslash\left(\Phi_{\sigma_{n}}^{u}\right)^{-1}\left(\mathbb{D}_{\widehat{r}}\right)$, so $H_{\sigma_{n}, t_{n}}\left(\varphi_{\sigma_{n}}^{u}\left(\gamma_{\sigma_{n}}^{\prime u}\right)\right) \subset \varphi_{\sigma_{n}, t_{n}}^{\xi}\left(P_{n} \backslash\right.$ $\left.G_{\sigma_{n}, t_{n}}\left(D_{t_{n}}\right)\right)$ and the same arguments as previously show that

$$
\text { if } \widetilde{z}_{n} \in H_{\sigma_{n}, t_{n}}\left(\varphi_{\sigma_{n}}^{u}\left(\gamma_{\sigma_{n}}^{\prime u}\right)\right) \text {, then } \widetilde{z}_{n} \rightarrow p_{a, b} .
$$

The above facts together with the almost uniform convergence imply that

$$
\begin{aligned}
\operatorname{cl} H_{\sigma_{n}, t_{n}}\left(\varphi_{\sigma_{n}}^{u}\left(\Gamma_{\sigma_{n}}^{u} \backslash \gamma_{\sigma_{n}}^{\prime \prime u}\right)\right) & \\
& =\operatorname{cl}\left(\varphi_{\sigma_{n}, t_{n}}^{\xi}\left(G_{\sigma_{n}, t_{n}}\left(\partial D_{t_{n}} \cap \mathbb{D}^{+}\right)\right) \cup H_{\sigma_{n}, t_{n}}\left(\varphi_{\sigma_{n}}^{u}\left(\gamma_{\sigma_{n}}^{\prime u}\right)\right)\right)
\end{aligned}
$$

tends to $\operatorname{cl} \varphi_{a, b}^{p}\left(G_{a, b}\left(\partial D_{1} \cap \mathbb{D}^{+}\right)\right)$in the Hausdorff metric.

Proof of Proposition 4.19. Lemmas 4.20 and 4.21 show that $\operatorname{cl}\left(\varphi_{a, b}^{p}\left(\Gamma_{a, b}^{p}\right)\right.$ $\left.\cup \varphi_{a, b}^{\infty}\left(\Gamma_{a, b}^{\infty}\right)\right)$ is a Jordan curve. Note that by almost uniform convergence, $\operatorname{cl} H_{\sigma_{n}, t_{n}}\left(\varphi_{\sigma_{n}}^{\infty}\left(\Gamma_{\sigma_{n}}^{\infty} \backslash\left(\gamma_{\sigma_{n}}^{\prime \infty} \cup \gamma_{\sigma_{n}}^{\prime \prime \infty}\right)\right)\right)=\operatorname{cl} \varphi_{\sigma_{n}, t_{n}}^{\infty}\left(\Gamma_{\sigma_{n}}^{\infty} \backslash\left(\gamma_{\sigma_{n}}^{\prime \infty} \cup \gamma_{\sigma_{n}}^{\prime \prime \infty}\right)\right)$ tends to $\operatorname{cl} \varphi_{a, b}^{\infty}\left(\Gamma_{a, b}^{\infty} \backslash\left(\gamma_{\sigma_{a, b}}^{\prime \infty} \cup \gamma_{\sigma_{a, b}}^{\prime \prime \infty}\right)\right)$ in the Hausdorff metric. This together with Lemmas 4.20, 4.21 and (27) implies that $\partial f_{\sigma_{n}, t_{n}}^{k}\left(U_{\sigma_{n}, t_{n}}\right)$ tends to $\operatorname{cl}\left(\varphi_{a, b}^{p}\left(\Gamma_{a, b}^{p}\right) \cup\right.$ $\left.\varphi_{a, b}^{\infty}\left(\Gamma_{a, b}^{\infty}\right)\right)$ in the Hausdorff metric.

An immediate consequence of Proposition 4.19 is

Corollary 4.22. The Jordan curve $\operatorname{cl}\left(\varphi_{a, b}^{p}\left(\Gamma_{a, b}^{p}\right) \cup \varphi_{a, b}^{\infty}\left(\Gamma_{a, b}^{\infty}\right)\right)$ depends continuously on $(a, b) \in \widetilde{\mathcal{U}}_{1}$ in the Hausdorff metric.

Now for $(a, b) \in \widetilde{\mathcal{U}}_{1}$ we define a topological disc $U_{a, b}$ such that $f_{a, b}^{k}$ is quadratic-like on $U_{a, b}$ and $\operatorname{cl} U_{\sigma_{n}, t_{n}}$ tends to $\mathrm{cl} U_{a, b}$ in the Hausdorff metric.

The case $(a, b) \in M_{1}$. Let $(a, b) \in M_{1}$ and take $\sigma_{n} \in M_{N}, t_{n} \rightarrow 1^{-}$such that $\left(a\left(\sigma_{n}, t_{n}\right), b\left(\sigma_{n}, t_{n}\right)\right) \rightarrow(a, b)$. For $\zeta \in\left\{p_{a, b}, \infty\right\}$ denote by $\widetilde{B}(\zeta)$ the unique component of $f_{a, b}^{-1}(B(\zeta)) \backslash B(\zeta)$. Consider the Blaschke product $h_{t}$ from $(26)$ for $t \in[0,1]$. Note that the forward trajectory of the $h_{t}$-critical point 0 is contained in $[0,1)$, so all inverse branches of $h_{t}^{-k}$ are defined on $\mathbb{D}^{+}$. Moreover, $\left.h_{t}\right|_{\partial \mathbb{D}}$ is a degree two covering depending continuously on $t \in[0,1]$. Therefore, there exists a branch $\nu_{t}$ of $h_{t}^{-k}$ defined on $\mathbb{D}^{+}$such that $\nu_{0}\left(\mathbb{D}^{+}\right)=\left\{r e^{2 \pi i \theta}: 0<r<1, \theta \in(\beta, \delta)\right\}$ for $\beta, \delta$ from the definition of $U_{\sigma}^{\prime}$ and $\nu_{t}$ depends continuously on $t \in[0,1]$. By (29) and the definition of the sets $U_{\sigma, t}$, we have

$$
\begin{aligned}
& \partial U_{\sigma_{n}, t_{n}} \cap B\left(\xi_{\sigma_{n}, t_{n}}\right)=\varphi_{\sigma_{n}, t_{n}}^{\xi}\left(\partial \nu_{t_{n}}\left(\mathbb{D}^{+} \backslash \operatorname{cl} D_{t_{n}}\right) \cap \mathbb{D}\right), \\
& \partial U_{\sigma_{n}, t_{n}} \cap \widetilde{B}\left(\xi_{\sigma_{n}, t_{n}}\right)=f_{\sigma_{n}, t_{n}}^{-1}\left(f_{\sigma_{n}, t_{n}}\left(\partial U_{\sigma_{n}, t_{n}} \cap B\left(\xi_{\sigma_{n}, t_{n}}\right)\right)\right) \cap \widetilde{B}\left(\xi_{\sigma_{n}, t_{n}}\right) .
\end{aligned}
$$


Now we observe that

$$
\begin{aligned}
& \operatorname{cl}\left(\varphi_{a, b}^{p}\left(\partial \nu_{1}\left(\mathbb{D}^{+} \backslash \operatorname{cl} D_{1}\right) \cap \mathbb{D}\right)\right. \\
& \cup\left(f_{a, b}^{-1}\left(f_{a, b}\left(\varphi_{a, b}^{p}\left(\partial \nu_{1}\left(\mathbb{D}^{+} \backslash \operatorname{cl} D_{1}\right) \cap \mathbb{D}\right)\right)\right) \cap \widetilde{B}\left(p_{a, b}\right)\right) \\
& \cup \varphi_{a, b}^{\infty}\left(\left(\varphi_{\sigma_{a, b}}^{\infty}\right)^{-1}\left(\partial U_{\sigma_{a, b}} \cap B_{\sigma_{a, b}}(\infty)\right)\right) \\
&\left.\cup\left(f_{a, b}^{-1}\left(f_{a, b}\left(\varphi_{a, b}^{\infty}\left(\left(\varphi_{\sigma_{a, b}}^{\infty}\right)^{-1}\left(\partial U_{\sigma_{a, b}} \cap B_{\sigma_{a, b}}(\infty)\right)\right)\right)\right) \cap \widetilde{B}(\infty)\right)\right)
\end{aligned}
$$

is a Jordan curve and $\partial U_{\sigma_{n}, t_{n}}$ converges to it in the Hausdorff metric. The proof is the same as the proof of Proposition 4.19 and we leave it to the reader.

Definition 4.23. For $(a, b) \in M_{1}$ let $U_{a, b}$ be the topological disc containing 1 , whose boundary is equal to the Jordan curve from (38).

By (34), we have $\operatorname{cl} \nu_{1}\left(\mathbb{D}^{+} \backslash \operatorname{cl} D_{1}\right) \subset \mathbb{D}^{+} \backslash \operatorname{cl} D_{1}$. This easily implies that $f_{a, b}^{k}$ is quadratic-like on $U_{a, b}$. Moreover, $\partial f_{a, b}^{k}\left(U_{a, b}\right)$ is equal to the Jordan curve from Proposition 4.19 (see Fig. 12).

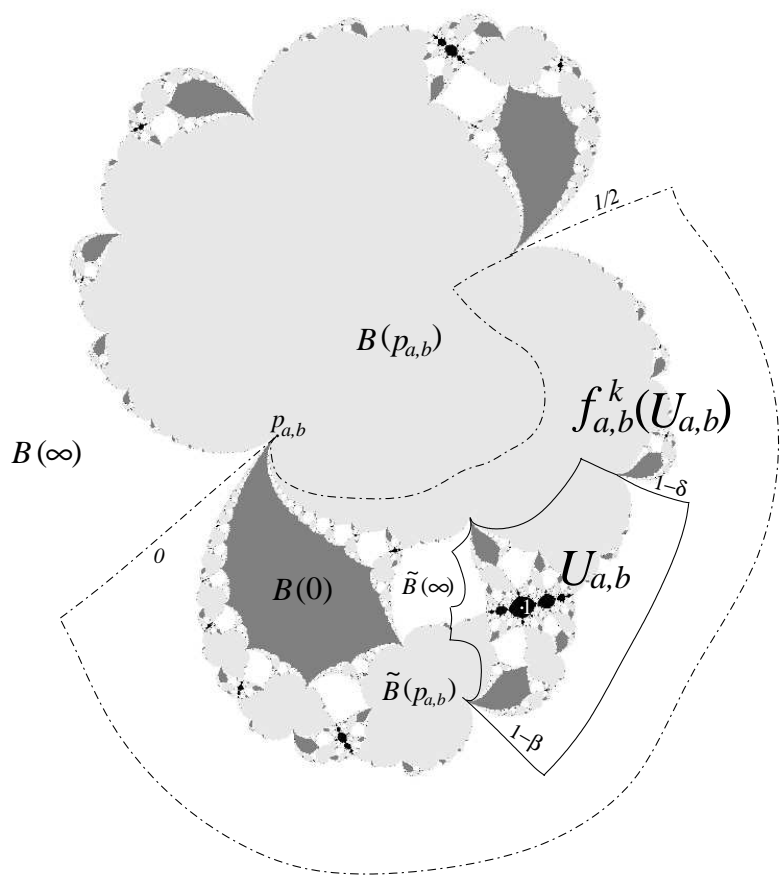

Fig. 12. The set $U_{a, b}$ for a parabolic map $f_{a, b}$

The case $(a, b) \notin M_{1}$. Now we extend the definition of $U_{a, b}$ for $(a, b) \in \widetilde{\mathcal{U}}_{1}$. 
Definition 4.24. For $(a, b) \in \widetilde{\mathcal{U}}_{1}$ let $A_{a, b}$ be the component of the complement of the Jordan curve $\operatorname{cl}\left(\varphi_{a, b}^{p}\left(\Gamma_{a, b}^{p}\right) \cup \varphi_{a, b}^{\infty}\left(\Gamma_{a, b}^{\infty}\right)\right)$ from Proposition 4.19 containing $f_{a, b}^{k}(1)$ and let $U_{a, b}$ be the component of $f_{a, b}^{-k}\left(A_{a, b}\right)$ containing 1 .

By (33), if we take sufficiently small $\varepsilon_{1}$ in the definition of $\widetilde{\mathcal{U}}_{1}$, then for $(a, b) \in \widetilde{\mathcal{U}}_{1}$ the point $f_{a, b}^{k}(1)$ is not in $\operatorname{cl}\left(\varphi_{a, b}^{p}\left(\Gamma_{a, b}^{p}\right) \cup \varphi_{a, b}^{\infty}\left(\Gamma_{a, b}^{\infty}\right)\right)$. Hence, $U_{a, b}$ is a topological disc, $f_{a, b}^{k}$ is quadratic-like on $U_{a, b}$ and $\mathrm{cl} U_{a, b}$ depends continuously on $(a, b) \in \widetilde{\mathcal{U}}_{1}$ by Corollary 4.22. Moreover, by Proposition 4.19, if $\sigma_{n} \in \mathcal{U}^{\prime}, t_{n} \rightarrow 1^{-}$such that $\left(a\left(\sigma_{n}, t_{n}\right), b\left(\sigma_{n}, t_{n}\right)\right) \rightarrow(a, b)$, then

$$
\operatorname{cl} U_{\sigma_{n}, t_{n}} \text { tends to } \operatorname{cl} U_{a, b} \text { in the Hausdorff metric. }
$$

Note that for $(a, b) \in M_{1}$ the definition of $U_{a, b}$ coincides with the previous one.

It is easy to check by (1) that for $\varrho \in \mathbb{C}$ we have

$$
\operatorname{Fix}(\varrho)=\{(a, b) \in(\widehat{\mathbb{C}} \times \widehat{\mathbb{C}}) \backslash \text { Sing }: P(\varrho, a, b)=0\},
$$

where $P$ is a polynomial such that $P(1, a, b)$ is equal to the left-hand side of (30). Hence, every $\left(a_{0}, b_{0}\right) \in \operatorname{Fix}(1)$ has an open neighbourhood

$$
\mathcal{W}_{a_{0}, b_{0}}=\mathbb{D}_{\varepsilon\left(a_{0}, b_{0}\right)}\left(a_{0}\right) \times \mathbb{D}_{\varepsilon\left(a_{0}, b_{0}\right)}\left(b_{0}\right) \subset \mathbb{C} \times \mathbb{C}
$$

such that

$$
\operatorname{Fix}(1) \cap \mathcal{W}_{a_{0}, b_{0}}=\left\{(a, b): a=\widetilde{\omega}_{1}(b), b \in \mathbb{D}_{\varepsilon\left(a_{0}, b_{0}\right)}\left(b_{0}\right)\right\}
$$

for some holomorphic map $\widetilde{\omega}_{1}$. By (40) and the implicit function theorem, for $\varrho \in \mathbb{C}$ close to 1 we have

$$
\operatorname{Fix}(\varrho) \cap \mathcal{W}_{a_{0}, b_{0}}=\left\{(a, b): a=\widetilde{\omega}_{\varrho}(b), b \in \mathbb{D}_{\varepsilon\left(a_{0}, b_{0}\right)}\left(b_{0}\right)\right\}
$$

for some holomorphic map $\widetilde{\omega}_{\varrho}$ depending holomorphically on $\varrho$. This implies that there exists a holomorphic homeomorphism

$$
\mathcal{H}_{a_{0}, b_{0}}: \mathcal{W}_{a_{0}, b_{0}} \rightarrow \mathbb{C} \times \mathbb{C}
$$

such that

$$
\begin{aligned}
\mathcal{H}_{a_{0}, b_{0}}\left(\widetilde{\mathcal{U}}_{1} \cap \mathcal{W}_{a_{0}, b_{0}}\right) & \subset\{(\eta, \mu): \eta=1\}, \\
\mathcal{H}_{a_{0}, b_{0}}\left(Q_{t}\left(\mathcal{U}^{\prime}\right) \cap \mathcal{W}_{a_{0}, b_{0}}\right) & \subset\left\{(\eta, \mu): \eta=\omega_{t}(\mu)\right\},
\end{aligned}
$$

where $\omega_{t}$ is a holomorphic map depending continuously on $t \leq 1$ close to 1 and $\omega_{1} \equiv 1$.

Lemma 4.25. There exists $c>0$ such that for every $t \in(0,1)$,

$$
\begin{aligned}
Q_{t}\left(\mathcal{U}^{\prime}\right) & \supset\left\{(a, b) \in \operatorname{Fix}(t): \operatorname{dist}\left((a, b), M_{t}\right)<c\right\}, \\
\widetilde{\mathcal{U}}_{1} & \supset\left\{(a, b) \in \operatorname{Fix}(1): \operatorname{dist}\left((a, b), M_{1}\right)<c\right\} .
\end{aligned}
$$

Proof. Note that the first statement of the lemma together with Lemma 4.12 and (42) implies the second one, so it is sufficient to prove the first 
statement. Let

$$
\mathcal{A}_{n}=\left\{\sigma \in \mathcal{U}^{\prime}: N_{\sigma}^{k j}(1) \in U_{\sigma}^{\prime} \text { for } 0 \leq j \leq n\right\} .
$$

It is easy to check that $\mathcal{A}_{n}$ is open in $\mathcal{U}^{\prime}$ and

$$
\partial \mathcal{A}_{n} \subset\left\{\sigma \in \mathcal{U}^{\prime}: N_{\sigma}^{k j}(1) \in U_{\sigma}^{\prime} \text { for } 0 \leq j \leq n-1, N_{\sigma}^{k n}(1) \in \partial U_{\sigma}^{\prime}\right\} .
$$

Since $\bigcap_{n \geq 0} \mathcal{A}_{n}=M_{N}$, we can find $n_{0}$ such that if $\mathcal{A}$ is the component of $\mathcal{A}_{n_{0}}$ containing $M_{N}$, then $\operatorname{cl} \mathcal{A} \subset \mathcal{U}^{\prime}$. It is easy to check that there exists a component $\gamma$ of $\partial \mathcal{A}$ such that the index of $\gamma$ with respect to a point from $M_{N}$ is equal to 1 . By (43) and the definition of $U_{\sigma, t}$,

$$
Q_{t}(\gamma) \subset\left\{(a(\sigma, t), b(\sigma, t)): f_{\sigma, t}^{k n_{0}}(1) \in \partial U_{\sigma, t}\right\}
$$

for $Q_{t}$ from Lemma 4.12. This together with (39) implies that any limit point of the sequence $Q_{t_{n}}\left(\sigma_{n}\right)$ for $\sigma_{n} \in \gamma, t_{n} \rightarrow 1^{-}$does not belong to $M_{1}$. Hence, by the compactness of $\gamma$ and the properties of $Q_{t}$, there exists $c>0$ such that $\operatorname{dist}\left(Q_{t}(\gamma), M_{t}\right)>c$ for every $t<1$. Note that by $(42)$ and the topological properties of surfaces we can assume that for some $\widetilde{c}>0$ the set $\left\{(a, b) \in \operatorname{Fix}(t): \operatorname{dist}\left((a, b), M_{t}\right)<\widetilde{c}\right\}$ for every $t<1$ is homeomorphic to an open subset of $\mathbb{C}$. Then repeating the proof of Lemma 4.12 with $\gamma_{1}$ replaced by $\gamma$ we show that $Q_{t}\left(\mathcal{U}^{\prime}\right) \supset\left\{(a, b) \in \operatorname{Fix}(t): \operatorname{dist}\left((a, b), M_{t}\right)<c\right\}$.

By Lemma 4.25 , if we take sufficiently small $\varepsilon_{1}$ in the definition of $\widetilde{\mathcal{U}}_{1}$, then $\widetilde{\mathcal{U}}_{1}$ is open in $\operatorname{Fix}(1)$, so we have

COROLlary 4.26. $\widetilde{\mathcal{U}}_{1}$ is a one-dimensional complex manifold containing $M_{1}$ and $\left\{\left.f_{a, b}^{k}\right|_{U_{a, b}}\right\}_{(a, b) \in \tilde{\mathcal{U}}_{1}}$ is an analytic family of quadratic-like maps.

Lemma 4.27. For every $\sigma \in M_{N}$ the curve $\{(a(\sigma, t), b(\sigma, t))\}_{t \in[0,1)}$ lands at a point in $M_{1}$.

Proof. Suppose that the curve has distinct limit points $\left(a_{1}, b_{1}\right),\left(a_{2}, b_{2}\right)$ $\in M_{1}$. By Lemma 4.25 , we can take a small ball $\mathcal{V}$ in $\mathbb{C} \times \mathbb{C}$ centred at $\left(a_{1}, b_{1}\right)$ such that $\left(a_{2}, b_{2}\right) \notin \operatorname{cl} \mathcal{V}$ and $\operatorname{cl} \mathcal{V} \cap \operatorname{Fix}(1) \subset \widetilde{\mathcal{U}}_{1}$. Note that if $\mathcal{V}$ is sufficiently small, then $\left\{\left.f_{a, b}^{k}\right|_{V_{a, b}}\right\}_{(a, b) \in \mathcal{V}}$ is an analytic family of quadraticlike maps, where $V_{a, b}$ is defined as the component of $f_{a, b}^{-k}\left(f_{a_{1}, b_{1}}^{k}\left(U_{a_{1}, b_{1}}\right)\right)$ containing 1. It is easy to check (see $[\mathrm{McM}]$ ) that if $(a, b) \in \mathcal{V} \cap \widetilde{\mathcal{U}}_{1}$, then the filled-in Julia set of $\left.f_{a, b}^{k}\right|_{U_{a, b}}$ and $\left.f_{a, b}^{k}\right|_{V_{a, b}}$ coincide. Similarly, by (39), if $(a, b)=(a(\sigma, t), b(\sigma, t)) \in \mathcal{V} \cap Q_{t}\left(\mathcal{U}^{\prime}\right)$, then the filled-in Julia sets of $\left.f_{a, b}^{k}\right|_{U_{\sigma, t}}$ and $\left.f_{a, b}^{k}\right|_{V_{a, b}}$ are the same.

By assumption, the curve $\{(a(\sigma, t), b(\sigma, t))\}_{t \in[0,1)}$ must intersect $\partial \mathcal{V}$ for infinitely many $t_{n}<1$ such that $t_{n} \rightarrow 1^{-}$. Passing to a subsequence, we can assume that $\left(a\left(\sigma, t_{n}\right), b\left(\sigma, t_{n}\right)\right)$ converges to some point in $\partial \mathcal{V} \cap \widetilde{\mathcal{U}}_{1}$. Since $\mathcal{V}$ can be arbitrarily small, this easily implies that $\left(a_{1}, b_{1}\right)$ is a point of density of the set of limit points of the curve $\{(a(\sigma, t), b(\sigma, t))\}_{t \in[0,1)}$. By 
the definition of $U_{\sigma, t}$, the map $\left.f_{\sigma, t}^{k}\right|_{U_{\sigma, t}}$ is hybrid equivalent to $z^{2}+\chi(\sigma)$. By Theorem 3.3 for the family $\left\{\left.f_{a, b}^{k}\right|_{V_{a, b}}\right\}_{(a, b) \in \mathcal{V}}$, the point $\left(a_{1}, b_{1}\right)$ is a point of density of the set of points $(a, b) \in \mathcal{V} \cap \widetilde{\mathcal{U}}_{1}$ for which $\left.f_{a, b}^{k}\right|_{V_{a, b}}$ is hybrid equivalent to $z^{2}+\chi(\sigma)$. Then Theorem 3.4 implies that all maps $\left.f_{a, b}^{k}\right|_{V_{a, b}}$ for $(a, b) \in \mathcal{V}$ are hybrid equivalent. Applying the same theorem to the family $\left\{\left.f_{a, b}^{k}\right|_{U_{a, b}}\right\}_{(a, b) \in \widetilde{\mathcal{U}}_{1}}$ we conclude that all maps $\left.f_{a, b}^{k}\right|_{U_{a, b}}$ for $(a, b)$ in the component of $\widetilde{\mathcal{U}}_{1}$ containing $\left(a_{1}, b_{1}\right)$ are hybrid equivalent. This is clearly impossible (as follows e.g. from Lemma 4.25).

By Lemma 4.27 , we can define a mapping

$$
Q_{1}: M_{N} \rightarrow M_{1}, \quad Q_{1}(\sigma)=\lim _{t \rightarrow 1^{-}} Q_{t}(\sigma) .
$$

Lemma 4.28. $Q_{1}$ is a homeomorphism.

Proof. By definition, $Q_{1}$ maps $M_{N}$ onto $M_{1}$. To show that $Q_{1}$ is injective, suppose that $Q_{1}\left(\sigma_{1}\right)=Q_{1}\left(\sigma_{2}\right)=\left(a_{1}, b_{1}\right)$. Define $\mathcal{V}$ and $V_{a, b}$ as in the proof of Lemma 4.27. Then Theorem 3.3 for the family $\left\{\left.f_{a, b}^{k}\right|_{V_{a, b}}\right\}_{(a, b) \in \mathcal{V}}$ shows $\sigma_{1}=\sigma_{2}$. Now we prove that $Q_{1}$ is continuous. Let $\sigma_{n} \rightarrow \sigma$ for $\sigma_{n}, \sigma \in M_{N}$ and suppose that

$$
\operatorname{dist}\left(Q_{1}\left(\sigma_{n}\right), Q_{1}(\sigma)\right)>c_{0}>0
$$

for all $n$. Take $0<c_{1}<c_{0}$. Since $Q_{t}$ is continuous, for any $j>0$ we can choose $n_{j}$ such that $\operatorname{dist}\left(Q_{1-1 / j}\left(\sigma_{n_{j}}\right), Q_{1-1 / j}(\sigma)\right)<c_{1}$. By (44), there exists $t_{j} \in(1-1 / j, 1)$ such that $\operatorname{dist}\left(Q_{t_{j}}\left(\sigma_{n_{j}}\right), Q_{t_{j}}(\sigma)\right)=c_{1}$. Passing to a subsequence, we can assume that $Q_{t_{j}}\left(\sigma_{n_{j}}\right) \rightarrow\left(a_{1}, b_{1}\right) \in M_{1}$ such that $\operatorname{dist}\left(\left(a_{1}, b_{1}\right), Q_{1}(\sigma)\right)=c_{1}$. Arguing as in the proof of Lemma 4.27 we show that $\left.f_{a_{1}, b_{1}}^{k}\right|_{U_{a_{1}, b_{1}}}$ is hybrid equivalent to $z^{2}+\chi(\sigma)$. Since $c_{1}$ was arbitrary, it follows that $Q_{1}(\sigma)$ is a point of density of the set of points $(a, b) \in M_{1}$ for which $\left.f_{a, b}^{k}\right|_{U_{a, b}}$ is hybrid equivalent to $z^{2}+\chi(\sigma)$. As in the proof of Lemma 4.27, this leads to a contradiction.

We have shown that $Q_{1}$ is a continuous injective map from $M_{N}$ onto $M_{1}$. By the compactness of $M_{N}, Q_{1}$ is a homeomorphism.

Since Fix(1) is a one-dimensional complex manifold, using Lemma 4.28 we can find a topological disc in Fix(1) containing $M_{1}$. This implies that the map $\widetilde{\omega}_{1}$ from (41) can be defined globally, i.e. there exist an open neighbourhood $\mathcal{W} \subset \mathbb{C} \times \mathbb{C}$ of $M_{1}$, a topological disc $W \subset \mathbb{C}$ and a holomorphic map $\widetilde{\omega}_{1}$ on $W$ such that

$$
\operatorname{Fix}(1) \cap \mathcal{W} \subset\left\{(a, b): a=\widetilde{\omega}_{1}(b), b \in W\right\} .
$$

If $\varepsilon_{1}$ in the definition of $\widetilde{\mathcal{U}}_{1}$ is sufficiently small, then $\widetilde{\mathcal{U}}_{1} \subset \mathcal{W}$ and by the implicit function theorem, there exists a biholomorphic map

$$
\mathcal{H}: \mathcal{W} \rightarrow \mathbb{C} \times \mathbb{C}
$$


such that

$$
\mathcal{H}\left(\widetilde{\mathcal{U}}_{1}\right) \subset\{(\eta, \mu): \eta=1\}, \quad \mathcal{H}\left(Q_{t}\left(\mathcal{U}^{\prime}\right) \cap \mathcal{W}\right) \subset\left\{(\eta, \mu): \eta=\omega_{t}(\mu)\right\}
$$

where $\omega_{t}$ is a holomorphic map depending continuously on $t \leq 1$ close to 1 and $\omega_{1} \equiv 1$. This together with Lemma 4.25 implies that the path of the topological discs $\mathcal{U}_{t}, t \in[0,1)$, from Corollary 4.13 can be extended continuously to $\mathcal{U}_{t}, t \in[0,1]$, i.e. there exist disc-equivalent Riemann surfaces $\mathcal{U}_{t} \subset \operatorname{Fix}(t), t \in[0,1]$, such that $\mathcal{U}_{0} \subset \mathcal{U}^{\prime}, M_{t} \subset \mathcal{U}_{t} \subset Q_{t}\left(\mathcal{U}^{\prime}\right)$ for $t \in[0,1)$, $M_{1} \subset \mathcal{U}_{1} \subset \widetilde{\mathcal{U}}_{1}$ and cl $\mathcal{U}_{t}$ depends continuously on $t \in[0,1]$ in the Hausdorff metric. Let

$$
\mathbf{f}_{t}=\left\{\left.f_{a, b}^{k}\right|_{U_{a, b}}\right\}_{(a, b) \in \mathcal{U}_{t}} \quad \text { for } t \in[0,1] .
$$

By Corollary 4.13, (39), Corollary 4.26, Theorem 3.3 and Proposition 3.5, the families $\mathbf{f}_{t}$ for $t \in[0,1]$ are Mandelbrot-like with the Mandelbrot-like set $M_{t}$ and $\left\{\mathbf{f}_{t}\right\}_{t \in[0,1]}$ is a continuous path of Mandelbrot-like families. By Proposition 3.7, the sets $M_{t}$ for $t \in[0,1]$ are quasiconformally homeomorphic.

4.4. The construction of $\mathbf{f}_{t}^{0}, \mathbf{f}_{t}^{\infty}$. To simplify notation, in the following two subsections we will use a new holomorphic system of coordinates $(\eta, \mu)$ in a part of the parameter space defined by $\mathcal{H}$ from (45). We write $f_{\eta, \mu}$ for $f_{a, b}$, where $\mathcal{H}(a, b)=(\eta, \mu)$. Recall that these coordinates are defined in some open neighbourhood of $\mathcal{U}_{1}$ in $\mathbb{C} \times \mathbb{C}$ and in these coordinates $\mathcal{U}_{1}$ is contained in the plane $\{\eta=1\}$. For simplicity, we write $\mu \in M_{1}, \mu \in \mathcal{U}_{1}$ instead of $(1, \mu) \in M_{1},(1, \mu) \in \mathcal{U}_{1}$ respectively.

For $\mu \in \mathcal{U}_{1}$ denote by $p_{\mu}$ the parabolic $f_{1, \mu}$-fixed point of multiplier 1 . For $\zeta \in\{0, \infty\}$ let $\Phi_{\eta, \mu}^{\zeta}$ be the Böttcher coordinates on $B(\zeta)$ for $f_{\eta, \mu}$. If $\mu \in M_{1}$, then $\Phi_{1, \mu}^{\zeta}$ is defined on $B(\zeta)$. Hence, if we fix $r_{0}<1$ close to 1 and a small $\widetilde{\varepsilon}>0$, and take $\mathcal{U}_{1}$ sufficiently close to $M_{1}$, then for $\mu \in \mathcal{U}_{1}$ and $\eta \in \mathbb{D}_{\widetilde{\varepsilon}}(1)$ the map $\left(\Phi_{\eta, \mu}^{\zeta}\right)^{-1}$ is defined on $\mathrm{cl} \mathbb{D}_{r_{0}}$ and $\left(\Phi_{\eta, \mu}^{\zeta}\right)^{-1}\left(\left[r_{0}^{2}, r_{0}\right]\right)$ is contained in a small neighbourhood of $p_{\mu}$. Let

$$
\gamma_{\eta, \mu}^{\zeta}(s)=\left(\Phi_{\eta, \mu}^{\zeta}\right)^{-1}(s) \quad \text { for } s \in\left[0, r_{0}\right] .
$$

For $\mu \in \mathcal{U}_{1}$ we can extend the curve $\gamma_{1, \mu}^{\zeta}$ taking its successive preimages by the branch of $f_{1, \mu}^{-1}$ fixing $p_{\mu}$ and parameterize it by $s \in[0,1)$ in such a way that $\gamma_{1, \mu}^{\zeta}\left(s^{2}\right)=f_{1, \mu}\left(\gamma_{1, \mu}^{\zeta}(s)\right)$. Then $\gamma_{1, \mu}^{\zeta}$ is a simple arc in $B(\zeta)$ beginning at $\zeta$ and landing at $p_{\mu}$. For $\eta \in \mathbb{D}_{\widetilde{\varepsilon}}(1)$ we can extend $\gamma_{\eta, \mu}^{\zeta}$ in the same way parameterizing it by $s \in[0, r]$ such that $r$ is arbitrarily close to 1 and $\gamma_{\eta, \mu}^{\zeta}(r)$ is arbitrarily close to $p_{\mu}$, if $\eta$ is sufficiently close to 1 . Since the Böttcher coordinates depend holomorphically on the mapping, the function $(\eta, \mu) \mapsto \gamma_{\eta, \mu}^{\zeta}(r)$ is holomorphic.

For $\mu \in \mathcal{U}_{1}$ consider the family $\left\{f_{\eta, \mu}\right\}_{\eta \in \mathbb{D}_{\tilde{\varepsilon}}(1)}$. Let $\eta(\lambda)=1+\lambda^{2}$ for $\lambda \in \mathbb{D}_{\sqrt{\widetilde{\varepsilon}}}$. Since $f_{1, \mu}$ has a double fixed point $p_{\mu}$, we can choose an $f_{\eta(\lambda), \mu^{-}}$ 
fixed point $\xi_{\mu}(\lambda)$ such that $\xi_{\mu}(0)=p_{\mu}$ and $(\lambda, \mu) \mapsto \xi_{\mu}(\lambda)$ is holomorphic. Let

$$
g_{\lambda, \mu}(z)=f_{\eta(\lambda), \mu}\left(z+\xi_{\mu}(\lambda)\right)-\xi_{\mu}(\lambda) .
$$

Then the family $\left\{g_{\lambda, \mu}\right\}_{\lambda \in \mathbb{D} \sqrt{\varepsilon}}$ satisfies the assumptions of Theorem I.4.21. Taking $\delta_{+}=\gamma_{\eta, \mu}^{\zeta}(r)$ in this theorem and repeating the proof of Theorem I.4.20 we show that for every $t \in(1,1+\varepsilon]$ with some small $\varepsilon>0$ there exists $\lambda_{\mu}^{\zeta}(t) \in \mathbb{D}_{\sqrt{\widetilde{\varepsilon}}}$ such that the curve $\gamma_{\eta\left(\lambda_{\mu}^{\zeta}(t)\right), \mu}^{\zeta}$ can be extended to a simple arc in $B(\zeta)$ connecting $\zeta$ to $f_{\eta\left(\lambda_{\mu}^{\zeta}(t)\right), \mu}(u)$, parameterized by $s \in[0,2-t]$ such that $\gamma_{\eta\left(\lambda_{\mu}^{\zeta}(t)\right), \mu}^{\zeta}\left(s^{2}\right)=f_{\eta\left(\lambda_{\mu}^{\zeta}(t)\right), \mu}\left(\gamma_{\eta\left(\lambda_{\mu}^{\zeta}(t)\right), \mu}^{\zeta}(s)\right)$,

$$
f_{\eta\left(\lambda_{\mu}^{\zeta}(t)\right), \mu}(u)=\gamma_{\eta\left(\lambda_{\mu}^{\zeta}(t)\right), \mu}^{\zeta}(2-t)
$$

and $\lambda_{\mu}^{\zeta}(t) \rightarrow 0$ as $t \rightarrow 1^{+}$. Moreover, by the proof of Theorem I.4.21 (see [DH1], Proposition XI.5) and by the holomorphicity of $(\eta, \mu) \mapsto \gamma_{\eta, \mu}^{\zeta}(r)$, we can choose the parameter $\lambda_{\mu}^{\zeta}(t)$ so that it depends continuously on $t \in$ $(1,1+\varepsilon]$ and holomorphically on $\mu \in \mathcal{U}_{1}$.

Let $\mathcal{G}_{\mu}^{\zeta}(1)=1$ and $\mathcal{G}_{\mu}^{\zeta}(t)=\eta\left(\lambda_{\mu}^{\zeta}(t)\right)$ for $t \in(1,1+\varepsilon]$. Then

$$
\mathcal{G}_{\mu}^{\zeta}:[1,1+\varepsilon] \rightarrow \mathbb{C}
$$

is a curve such that

$$
f_{\mathcal{G}_{\mu}^{\zeta}(t), \mu}(u)=\gamma_{\mathcal{G}_{\mu}^{\zeta}(t), \mu}^{\zeta}(2-t)
$$

for $t \in(1,1+\varepsilon]$. In particular, $u \in B(\zeta)$ for the map $f_{\mathcal{G}_{\mu}^{\zeta}(t), \mu}$. Note that if $\Phi_{\mathcal{G}_{\mu}^{\zeta}(t), \mu}^{\zeta}$ is defined in a neighbourhood of the curve $\gamma_{\mathcal{G}_{\mu}^{\zeta}(t), \mu}^{\zeta}($ e.g. if $1 \notin B(\zeta))$, then $\gamma_{\mathcal{G}_{\mu}^{\zeta}(t), \mu}^{\zeta}(s)=\left(\Phi_{\mathcal{G}_{\mu}^{\zeta}(t), \mu}^{\zeta}\right)^{-1}(s)$ for every $s \in[0,2-t]$, so

$$
\Phi_{\mathcal{G}_{\mu}^{\zeta}(t), \mu}^{\zeta}\left(f_{\mathcal{G}_{\mu}^{\zeta}(t), \mu}(u)\right)=2-t .
$$

For $t \in[1,1+\varepsilon]$ let

$$
\mathcal{U}_{t}^{\zeta}=\left\{\left(\mathcal{G}_{\mu}^{\zeta}(t), \mu\right): \mu \in \mathcal{U}_{1}\right\} .
$$

Since $\mu \mapsto \lambda_{\mu}^{\zeta}(t)$ is holomorphic, $\mu \mapsto \mathcal{G}_{\mu}^{\zeta}(t)$ is holomorphic, so $\mathcal{U}_{t}^{\zeta}$ are disc-equivalent Riemann surfaces. For $(\eta, \mu) \in \mathcal{U}_{t}^{\zeta}$ define $U_{\eta, \mu}$ to be the component of $f_{\eta, \mu}^{-k}\left(f_{1, \mu}^{k}\left(U_{1, \mu}\right)\right)$ containing 1 . If $\varepsilon$ is sufficiently small, then $U_{\eta, \mu}$ is a topological disc, $f_{\eta, \mu}^{k}$ is quadratic-like on $U_{\eta, \mu}$ and

$$
\mathbf{f}_{t}^{\zeta}=\left\{\left.f_{\eta, \mu}^{k}\right|_{U_{\eta, \mu}}\right\}_{(\eta, \mu) \in \mathcal{U}_{t}^{\zeta}}
$$

is an analytic family of quadratic-like maps. By Theorem 3.3 and Proposition 3.5, this is a Mandelbrot-like family and $\left\{\mathbf{f}_{t}^{\zeta}\right\}_{t \in[1,1+\varepsilon]}$ is a continuous path of Mandelbrot-like families such that $\mathbf{f}_{1}^{\zeta}=\mathbf{f}_{1}$. Let $M_{t}^{\zeta}$ be the 
Mandelbrot-like set in $\mathcal{U}_{t}^{\zeta}$. By Proposition 3.7, all the sets $M_{t}^{\zeta}$ are quasiconformally homeomorphic.

4.5. $B(\infty)$ is exotic, $B(0)$ is not exotic. Now we show that for $t>1$, if $(\eta, \mu) \in \mathcal{U}_{t}^{\infty}$, then $B(\infty)$ for the map $f_{\eta, \mu}$ is not simply connected and if $(\eta, \mu) \in M_{t}^{0}$, then $B(0)$ for the map $f_{\eta, \mu}$ is simply connected.

Let $\mu \in \mathcal{U}_{1}$ and consider the curve

$$
\operatorname{cl}\left(\varphi_{a, b}^{p}\left(G_{a, b}\left(\left[h_{1}(0), 1\right)\right)\right) \cup \gamma_{1, \mu}^{\infty},\right.
$$

where $(a, b)$ is such that $\mathcal{H}(a, b)=(1, \mu)$ for $\mathcal{H}$ from $(45)$ and $\gamma_{1, \mu}^{\infty}$ is the curve from Subsection 4.4. Then the curve (47) is contained in $B(\infty) \cup\left\{p_{\mu}\right\} \cup B\left(p_{\mu}\right)$ and connects $\infty$ to $f_{1, \mu}(u)$. Moreover, one of the two components of the preimage under $f_{1, \mu}^{-1}$ of this curve is equal to the Jordan curve

$$
\operatorname{cl}\left(\varphi_{a, b}^{p}\left(G_{a, b}((-1,1))\right) \cup \varphi_{a, b}^{\infty}\left(\gamma_{\sigma_{a, b}}^{\prime \infty} \cup\left(\Phi_{\sigma_{a, b}}^{\infty}\right)^{-1}((-\widehat{r}, \widehat{r})) \cup \gamma_{\sigma_{a, b}}^{\prime \prime \infty}\right)\right)
$$

for $\gamma_{\sigma_{a, b}}^{\prime \infty}, \gamma_{\sigma_{a, b}}^{\prime \prime \infty}$ from (24), which is contained in $B(\infty) \cup f_{1, \mu}^{-1}\left(p_{\mu}\right) \cup B\left(p_{\mu}\right)$, contains $\infty, p_{\mu}, u$ and separates 0 from $f_{1, \mu}^{-1}(0) \backslash\{0\}$.

Let $(\eta, \mu) \in \mathcal{U}_{t}^{\infty}$ for $t>1$. Recall that the curve $\gamma_{\eta, \mu}^{\infty}$ from Subsection 4.4 is contained in $B(\infty)$ for $f_{\eta, \mu}$ and connects $\infty$ to $f_{\eta, \mu}(u)$. Moreover, the proof of Theorem I.4.20 applied for the family $\left\{f_{\eta, \mu}\right\}_{\eta \in \mathbb{E}_{\tilde{\varepsilon}}(1)}$ shows that $\gamma_{\eta, \mu}^{\infty}$ is arbitrarily close to the curve (47), if $t$ is sufficiently close to 1 . Taking the component of the preimage under $f_{\eta, \mu}^{-1}$ of $\gamma_{\eta, \mu}^{\infty}$ which is close to (48) we find a Jordan curve in $B(\infty)$ for $f_{\eta, \mu}$ separating 0 from $f_{\eta, \mu}^{-1}(0) \backslash\{0\}$. Hence, $B(\infty)$ is not simply connected for $(\eta, \mu) \in \mathcal{U}_{t}^{\infty}$.

Consider now a map $f_{1, \mu}$ for $\mu \in M_{1}$ and let $K_{\mu}$ be the filled-in Julia set of the quadratic-like map $\left.f_{1, \mu}^{k}\right|_{U_{1, \mu}}$. By the definition of the set $U_{\sigma, t}$, it is easy to check that

$$
K_{\mu} \ni l_{\infty}(\alpha), \tilde{l}_{\infty}(\alpha),
$$

where $\alpha$ is Head's angle used in the definition of $U_{\sigma}^{\prime}$ and $l_{\infty}(\alpha), \widetilde{l}_{\infty}(\alpha)$ denote the landing points of the suitable rays for the map $f_{1, \mu}$. Note that $l_{\infty}(\alpha)$ is a fixed repelling point for the quadratic-like map $f_{1, \mu}^{k} \mid U_{1, \mu}$. Hence, for the map $f_{\eta, \mu}$ with $(\eta, \mu) \in M_{t}^{0}$ and $t>1$ close to 1 we have

$$
K_{\eta, \mu} \ni l_{\infty}(\alpha), \tilde{l}_{\infty}(\alpha),
$$

where $K_{\eta, \mu}$ is the filled-in Julia set of $\left.f_{\eta, \mu}^{k}\right|_{U_{\eta, \mu}}$ and $l_{\infty}(\alpha), \widetilde{l}_{\infty}(\alpha)$ are the suitable landing points for the map $f_{\eta, \mu}$. Since $K_{\eta, \mu}$ is connected, this implies that the set $B(\infty) \cup K_{\eta, \mu} \cup \widetilde{B}(\infty)$ for $f_{\eta, \mu}$ is connected, so

$$
C=\mathrm{cl} \bigcup_{n=-\infty}^{\infty} f_{\eta, \mu}^{n}\left(B(\infty) \cup K_{\eta, \mu}\right)
$$

is connected. By the classification theorem, since only one $f_{\eta, \mu}$-critical point 1 is not contained in $B(0) \cup B(\infty)$, the closure of any Fatou component 
different from a component of the entire basin of attraction to 0 or $\infty$ must contain points from the forward trajectory of $1 \in K_{\eta, \mu}$. This together with the connectedness of $C$ easily implies that the complement of the entire basin of attraction to 0 is connected. Moreover, by Theorem I.4.4, $B(0)$ is completely invariant, i.e. $B(0)$ is the entire basin of attraction to 0 . We conclude that $\widehat{\mathbb{C}} \backslash B(0)$ is connected, which means that $B(0)$ is simply connected.

REMARK. Note that it is not true that $B(0)$ is simply connected for every $\mu \in \mathcal{U}_{t}^{0}$. In fact, if $1, u \in B(0)$, then there are three critical points in $B(0)$, so $B(0)$ cannot be simply connected.

Recall that an exotic basin for a cubic rational map is a non-simply connected completely invariant basin of attraction containing less than three critical points counted with multiplicity (for more information see [P1], [Ba]). Let $(\eta, \mu) \in M_{t}^{\infty}$. Then $B(\infty)$ for the map $f_{\eta, \mu}$ is not simply connected and contains two critical points counted with multiplicity. Moreover, by Theorem I.4.4, $B(\infty)$ is completely invariant. Hence, $B(\infty)$ is exotic.

Consider now $(\eta, \mu) \in M_{t}^{0}$. We have shown that $B(0)$ for the map $f_{\eta, \mu}$ is simply connected. Thus, there exists a topological disc $V \subset B(0)$ such that $0, u \in V$ and $f_{\eta, \mu}^{-1}(V)$ is a topological disc containing $\mathrm{cl} V$. If we perturb slightly $(\eta, \mu) \in \mathcal{U}_{t}^{0}$, then $V$ has the same properties for the perturbed map $f_{\eta, \mu}$. If $1 \in B(0)$ for $f_{\eta, \mu}$, then $B(0)$ is not exotic by definition. If $1 \notin B(0)$, then $B(0)=\bigcup_{n \geq 0} f_{\eta, \mu}^{-n}(V)$, so $B(0)$ is simply connected as the union of an increasing sequence of topological discs. Hence, if we take $\mathcal{U}_{1}$ sufficiently close to $M_{1}$ and $\varepsilon$ sufficiently close to 0 , then $B(0)$ is not exotic for $(\eta, \mu) \in \mathcal{U}_{t}^{0}$.

4.6. Extending the path $\left\{\mathbf{f}_{t}^{0}\right\}$. We only sketch the proof, since it is similar to the construction of the families $\mathbf{f}_{t}$ for $0<t<1$. Return to the standard coordinates $(a, b)$ in the parameter space and consider the family $\mathbf{f}_{1+\varepsilon}^{0}$. To simplify notation, parameterize the topological disc $\mathcal{U}_{1+\varepsilon}^{0}$ by $\tau$ in some topological disc in $\mathbb{C}$ and write $f_{\tau}$ instead of $f_{a, b}$ for $(a, b) \in \mathcal{U}_{1+\varepsilon}^{0}$.

If $\tau \in M_{1+\varepsilon}^{0}$, then $1 \notin B(0)$ and $f_{\tau}$ on $B(0)$ is conjugate to a cubic Blaschke product with a supersink. Hence, there exists a topological disc $\widetilde{V}_{\tau} \subset B(0)$ with smooth Jordan boundary depending continuously on $\tau \in$ $M_{1+\varepsilon}^{0}$ in the Hausdorff metric such that $0, u \in \widetilde{V}_{\tau}$ and there exists a simply connected component $V_{\tau}$ of $f_{\tau}^{-1}\left(\widetilde{V}_{\tau}\right)$ such that $\operatorname{cl} \widetilde{V}_{\tau} \subset V_{\tau}$. Then $f_{\tau}$ is a cubic cover on the closed annulus $\operatorname{cl} V_{\tau} \backslash \widetilde{V}_{\tau}$.

Note that diminishing the sets $\mathcal{U}_{t}^{0}, 1<t \leq 1+\varepsilon$, we can assume that $\mathcal{U}_{1+\varepsilon}^{0}$ is arbitrarily close to $M_{1+\varepsilon}^{0}$. Hence, for $\tau \in \mathcal{U}_{1+\varepsilon}^{0}$ we can find a topological disc $\widetilde{V}_{\tau} \subset B(0)$ with smooth Jordan boundary depending continuously on $\tau \in \mathcal{U}_{1+\varepsilon}^{0}$ with the same properties as above.

For $\tau \in \mathcal{U}_{1+\varepsilon}^{0}$ let

$$
\psi_{\tau}: \mathbb{D} \rightarrow V_{\tau}
$$


be the unique Riemann mapping such that $\psi_{\tau}(0)=0$ and $\psi_{\tau}^{\prime}(0)>0$. Fix $0<r_{0}<1$. We define a $C^{1}$-smooth cubic branched cover

$$
g_{\tau}: \mathbb{D} \rightarrow \mathbb{D}
$$

depending continuously on $\tau$ such that $g_{\tau}(z)=z^{3}$ on $\mathbb{D} \backslash \mathbb{D} \sqrt{r_{0}}$ and $g_{\tau}(z)=$ $r_{0} \psi_{\tau}^{-1}\left(f_{\tau}\left(\psi_{\tau}\left(z / r_{0}\right)\right)\right)$ on $\mathbb{D}_{r_{0}}$. Applying the measurable Riemann theorem for a suitable $g_{\tau}$-invariant conformal structure on $\mathbb{D}$ we obtain a continuous family of quasiconformal homeomorphisms $H_{\tau}$ of $\mathbb{D}$, holomorphic on $\mathbb{D}_{r_{0}}$ such that $H_{\tau}(0)=0$ and $H_{\tau} \circ g_{\tau} \circ H_{\tau}^{-1}$ is a cubic Blaschke product of the form

$$
h_{\alpha}(z)=z^{2} \frac{z-\alpha}{1-\bar{\alpha} z}
$$

for some $\alpha=\alpha(\tau) \in \mathbb{D}$ (having no relation to Head's angle). Let $v_{\alpha}$ be the unique non-zero $h_{\alpha}$-critical point in $\mathbb{D}$ and let $\Phi_{\alpha}$ (resp. $\Phi_{\tau}$ ) be the Böttcher coordinates for $h_{\alpha}$ on $\mathbb{D}$ (resp. $f_{\tau}$ on $B(0)$ ). If we take $\mathcal{U}_{1+\varepsilon}^{0}$ sufficiently close to $M_{1+\varepsilon}^{0}$, then $\Phi_{\tau}$ is defined in a neighbourhood of the curve $\gamma_{\eta, \mu}^{0}$ from Subsection 4.4 for $(\eta, \mu)$ corresponding to $\tau$, so by (46) we have

$$
\Phi_{\tau}\left(f_{\tau}(u)\right)=1-\varepsilon \quad \text { for } \tau \in \mathcal{U}_{1+\varepsilon}^{0} .
$$

For $\alpha \in \mathbb{D}$ set

$$
\Psi(\alpha)=\Phi_{\alpha}\left(h_{\alpha}\left(v_{\alpha}\right)\right) .
$$

Since $f_{\tau}$ on $\widetilde{V}_{\tau}$ is holomorphically conjugate to $h_{\alpha(\tau)}$ by

$$
G_{\tau}(z)=H_{\tau}\left(r_{0} \psi_{\tau}^{-1}(z)\right)
$$

it follows from the uniqueness of the Böttcher coordinates that

$$
\Phi_{\alpha(\tau)} \circ G_{\tau}=\Phi_{\tau}, \quad \Psi(\alpha(\tau))=1-\varepsilon .
$$

In the same way as in the proof of Theorem I.4.14 we show that $\alpha \mapsto \Psi(\alpha)$ is a local homeomorphism for $\alpha \in \mathbb{D} \backslash\{0\}$. Since $\tau \mapsto \alpha(\tau)$ is continuous, this together with (50) implies that in fact there exists $\alpha_{0} \in \mathbb{D}$ such that

$$
\alpha(\tau)=\alpha_{0} \quad \text { for every } \tau \in \mathcal{U}_{1+\varepsilon}^{0} .
$$

It is easy to check that if we take $\mathcal{U}_{1+\varepsilon}^{0}$ sufficiently close to $M_{1+\varepsilon}^{0}$, then we can assume

$$
G_{\tau}\left(\widetilde{V}_{\tau}\right) \supset \mathbb{D}_{r_{1}}
$$

for some fixed $r_{1}<1$ arbitrarily close to 1 .

Now define $\mathbf{f}_{t}^{0}$ for $t \in[1+\varepsilon, 1+2 \varepsilon]$, setting

$$
\mathcal{U}_{t}^{0}=\mathcal{U}_{1+\varepsilon}^{0}, \quad \mathbf{f}_{t}^{0}=\left\{\left.f_{\tau}^{k}\right|_{U_{\tau}^{t}}\right\}_{\tau \in \mathcal{U}_{t}^{0}}
$$

for topological discs $U_{\tau}^{t}$ such that $1 \in U_{\tau}^{t}, f_{\tau}^{k}$ is quadratic-like on $U_{\tau}^{t}, t \mapsto \partial U_{\tau}^{t}$ is continuous in the Hausdorff metric, $U_{\tau}^{1+\varepsilon}=U_{\tau}, U_{\tau}^{t_{1}} \supset U_{\tau}^{t_{2}}$ for every 
$t_{1}<t_{2}$ and

$$
f_{\tau}^{k}\left(U_{\tau}^{1+2 \varepsilon}\right) \cap V_{\tau}=\emptyset \quad \text { for } \tau \in \mathcal{U}_{1+2 \varepsilon}^{0} .
$$

It is clear that we can find such sets $U_{\tau}^{t}$ by taking successive preimages of the sets $U_{\tau}$ under $\left(\left.f_{\tau}^{k}\right|_{U_{\tau}}\right)^{-1}$. Moreover, it is obvious that $\left\{\mathbf{f}_{t}^{0}\right\}_{t \in[1+\varepsilon, 1+2 \varepsilon]}$ is a Mandelbrot-like path.

Fix some $r \in\left(\left|\alpha_{0}\right|, 1\right)$. By (51), we can assume that $v_{\alpha} \in \mathbb{D}_{r_{1}}$ for every $\alpha \in \mathbb{D}_{r}$, so $h_{\alpha}$ is a cubic cover on the closed annulus $\operatorname{cl}\left(h_{\alpha}^{-1}\left(\mathbb{D}_{r_{1}}\right) \cap \mathbb{D}\right) \backslash \mathbb{D}_{r_{1}}$. This enables us to define for $\alpha \in \mathbb{D}_{r}$ and $\tau \in \mathcal{U}_{1+2 \varepsilon}^{0}$ a $C^{1}$-smooth cubic branched cover $g_{\tau, \alpha}$ of $\widehat{\mathbb{C}}$ depending continuously on $\tau, \alpha$ such that $g_{\tau, \alpha}=f_{\tau}$ on $\widehat{\mathbb{C}} \backslash G_{\tau}^{-1}\left(h_{\alpha}^{-1}\left(\mathbb{D}_{r_{1}}\right) \cap \mathbb{D}\right), g_{\tau, \alpha}=G_{\tau}^{-1} \circ h_{\alpha} \circ G_{\tau}$ on $G_{\tau}^{-1}\left(\mathbb{D}_{r_{1}}\right)$ and $g_{\tau, \alpha_{0}}=f_{\tau}$. As previously, apply the measurable Riemann theorem for suitable $g_{\tau, \alpha^{-}}$ invariant conformal structures to obtain quasiconformal homeomorphisms $H_{\tau, \alpha}$ of $\widehat{\mathbb{C}}$ such that $H_{\tau, \alpha}$ is holomorphic on $G_{\tau}^{-1}\left(\mathbb{D}_{r_{1}}\right)$ and $H_{\tau, \alpha} \circ g_{\tau, \alpha} \circ H_{\tau, \alpha}^{-1}=$ $f_{a(\tau, \alpha), b(\tau, \alpha)}$ for some continuous functions $a(\tau, \alpha), b(\tau, \alpha)$. Write $f_{\tau, \alpha}$ for $f_{a(\tau, \alpha), b(\tau, \alpha)}$ and let

$$
U_{\tau, \alpha}=H_{\tau, \alpha}\left(U_{\tau}^{1+2 \varepsilon}\right) .
$$

Since $f_{\tau}^{k}\left(U_{\tau}^{1+2 \varepsilon}\right) \cap V_{\tau}=\emptyset$, we have $\left.f_{\tau}^{k}\right|_{U_{\tau}^{1+2 \varepsilon}}=\left.g_{\tau, \alpha}^{k}\right|_{U_{\tau}^{1+2 \varepsilon}}$. Hence, $U_{\tau, \alpha}$ is a topological disc containing 1 and $f_{\tau, \alpha}^{k}$ is quadratic-like on $U_{\tau, \alpha}$. Moreover, $f_{\tau, \alpha_{0}}=f_{\tau}, U_{\tau, \alpha_{0}}=U_{\tau}^{1+2 \varepsilon}$ and $\left.f_{\tau, \alpha}^{k}\right|_{U_{\tau, \alpha}}$ is hybrid equivalent to $\left.f_{\tau}^{k}\right|_{U_{\tau}^{1+2 \varepsilon} \text {. }}$

For $\tau \in \mathcal{U}_{1+2 \varepsilon}^{0}$ and $\alpha \in \mathbb{D}_{r}$ let $Q(\tau, \alpha)=(a(\tau, \alpha), b(\tau, \alpha))$. Define also $Q_{\alpha}: \mathcal{U}_{1+2 \varepsilon}^{0} \rightarrow \mathbb{C} \times \mathbb{C}$ and $Q_{\tau}: \mathbb{D}_{r} \rightarrow \mathbb{C} \times \mathbb{C}$ setting $Q_{\alpha}(\tau)=Q_{\tau}(\alpha)=Q(\tau, \alpha)$. Let $\Phi_{\tau, \alpha}$ be the Böttcher coordinates for $f_{\tau, \alpha}$ on $B(0)$ and let

$$
\widehat{\Psi}(\tau, \alpha)=\Phi_{\tau, \alpha}\left(f_{\tau, \alpha}(u)\right) .
$$

It is easy to check that

$$
\widehat{\Psi}(\tau, \alpha)=\Psi(\alpha) .
$$

Since $\alpha \mapsto \Psi(\alpha)$ is a local homeomorphism for $\alpha \neq 0$, this implies that for every $\alpha_{1} \in \mathbb{D}_{r} \backslash\{0\}$ we have $Q_{\alpha_{1}}\left(\mathcal{U}_{1+2 \varepsilon}^{0}\right) \cap Q_{\alpha_{2}}\left(\mathcal{U}_{1+2 \varepsilon}^{0}\right)=\emptyset$ if $\alpha_{2}$ is sufficiently close to $\alpha_{1}$. Moreover, for every $\alpha \in \mathbb{D}_{r}$ the map $Q_{\alpha}$ is a homeomorphism on $M_{1+2 \varepsilon}^{0}$ and $Q_{\alpha}\left(M_{1+2 \varepsilon}^{0}\right)$ is disjoint from $Q_{\alpha}\left(\mathcal{U}_{1+2 \varepsilon}^{0} \backslash M_{1+2 \varepsilon}^{0}\right)$. Let

$$
\begin{aligned}
& \mathcal{X}=\left\{(\tau, \alpha): \tau \in \mathcal{U}_{1+2 \varepsilon}^{0}, \alpha \in \mathbb{D}_{r} \backslash\{0\}\right\}, \\
& \mathcal{Y}=\left\{(\tau, \alpha): \tau \in M_{1+2 \varepsilon}^{0}, \alpha \in \mathbb{D}_{r} \backslash\{0\}\right\} .
\end{aligned}
$$

We observe that $Q(\mathcal{X})$ contains an open set in $\mathbb{C} \times \mathbb{C}$ containing $Q(\mathcal{Y})$. (The proof is the same as for Lemma 4.12, where we replace the indices of curves by indices of $Q$ restricted to suitable three-dimensional manifolds (boundaries of open subsets of $\mathbb{C} \times \mathbb{C}$ ) homeomorphic to the threedimensional sphere $S^{3}$.) This implies that $\left\{\left.f_{\tau, \alpha}^{k}\right|_{U_{\tau, \alpha}}\right\}_{(a(\tau, \alpha), b(\tau, \alpha)) \in \operatorname{int} Q(\mathcal{X})}$ is an analytic family of quadratic-like maps. Moreover, by Theorem 3.3, for every $\tau \in M_{1+2 \varepsilon}^{0}$ the set $Q_{\tau}\left(\mathbb{D}_{r} \backslash\{0\}\right)$ is analytic. These facts imply that 
$Q_{\tau}\left(\mathbb{D}_{r} \backslash\{0\}\right)$ is a Riemann surface for $\tau \in M_{1+2 \varepsilon}^{0}$. In the same way as in the proof of Theorem I.4.14 we show that the map $\widehat{\Psi}$ has no critical points in $Q_{\tau}\left(\mathbb{D}_{r} \backslash\{0\}\right)$ for $\tau \in M_{1+2 \varepsilon}^{0}$. This together with the analyticity of the sets $\{\widehat{\Psi}=$ const $\}$ implies that the sets $Q_{\alpha}\left(\mathcal{U}_{1+2 \varepsilon}^{0}\right)$ for $\alpha \in \mathbb{D}_{r} \backslash\{0\}$ contain discequivalent Riemann surfaces containing $Q_{\alpha}\left(M_{1+2 \varepsilon}^{0}\right)$. Note that for $\alpha=0$ the map $f_{\tau, 0}$ has a double critical point at 0 , so by $(2)$, the set $Q_{0}\left(\mathcal{U}_{1+2 \varepsilon}^{0}\right)$ is contained in the plane $\{(a, b): b=1 / 2\}$. Repeating the proof of Lemma 4.12 we show that $Q_{0}\left(\mathcal{U}_{1+2 \varepsilon}^{0}\right)$ contains a topological disc containing $Q_{0}\left(M_{1+2 \varepsilon}^{0}\right)$. Therefore, for $\alpha \in \mathbb{D}_{r}$ we can find topological discs $\widetilde{\mathcal{U}}_{\alpha}^{0} \subset Q_{\alpha}\left(\mathcal{U}_{1+2 \varepsilon}^{0}\right)$ such that $Q_{\alpha}\left(M_{1+2 \varepsilon}^{0}\right) \subset \widetilde{\mathcal{U}}_{\alpha}^{0}, \operatorname{cl} \widetilde{\mathcal{U}}_{\alpha}^{0}$ depends continuously on $\alpha$ in the Hausdorff metric and $\widetilde{\mathcal{U}}_{\alpha_{0}}^{0}=\mathcal{U}_{1+2 \varepsilon}^{0}$. Then $\left\{\left.f_{a, b}^{k}\right|_{U_{a, b}}\right\}_{(a, b) \in \tilde{\mathcal{U}}_{\alpha}^{0}}$ is a Mandelbrot-like family for $U_{a, b}=U_{\tau, \alpha}$ with the Mandelbrot-like set $Q_{\alpha}\left(M_{1+2 \varepsilon}^{0}\right)$.

Now for $t \in(1+2 \varepsilon, 2]$ we can define

$$
\mathbf{f}_{t}^{0}=\left\{\left.f_{a, b}^{k}\right|_{U_{a, b}}\right\}_{(a, b) \in \mathcal{U}_{t}^{0}} \quad \text { for } \mathcal{U}_{t}^{0}=\widetilde{\mathcal{U}}_{\alpha_{0}(2-t) /(1-2 \varepsilon)}^{0} .
$$

It is clear that the path $\left\{\mathbf{f}_{t}^{0}\right\}_{t \in[1,2]}$ is the suitable Mandelbrot-like path. This finishes the proof of Theorem 4.1.

\section{References}

[Ba] K. Barański, From Newton's method to exotic basins. Part I: The parameter space, Fund. Math. 158 (1998), 249-288.

[CGS] J. H. Curry, L. Garnett and D. Sullivan, On the iteration of a rational function: computer experiments with Newton's method, Comm. Math. Phys. 91 (1983), $267-277$.

[DH1] A. Douady et J. H. Hubbard, Etude dynamique des polynômes complexes, I et II, avec la collaboration de P. Lavours, Tan Lei et P. Sentenac, Publ. d'Orsay 84-02, 85-04, 1984-1985.

[DH2] - - - On the dynamics of polynomial-like mappings, Ann. Sci. École Norm. Sup. (4) 18 (1985), 287-343.

[Ma] P. Makienko, Pinching and plumbing deformations of quadratic rational maps, preprint, Internat. Centre Theoret. Phys., Miramare-Trieste, 1993.

[McM] C. T. McMullen, Complex Dynamics and Renormalization, Ann. of Math. Stud. 135, Princeton Univ. Press, 1994.

[Mi] J. Milnor, Dynamics in one complex variable: introductory lectures, preprint, SUNY at Stony Brook, IMS \# 1990/5.

[P1] F. Przytycki, Iterations of rational functions: which hyperbolic components contain polynomials?, Fund. Math. 149 (1996), 95-118.

[P2] - Remarks on simple-connectedness of basins of sinks for iterations of rational maps, in: Banach Center Publ. 23, PWN, 1989, 229-235.

[Ro] P. Roesch, Topologie locale des méthodes de Newton cubiques, Ph.D. thesis, École Norm. Sup. de Lyon, 1997.

[Sh] M. Shishikura, The connectivity of the Julia set of rational maps and fixed points, preprint, Inst. Hautes Études Sci., Bures-sur-Yvette, 1990. 
[St] N. Steinmetz, Rational Iteration, de Gruyter, Berlin, 1993.

[Ta] L. Tan, Branched coverings and cubic Newton maps, Fund. Math. 154 (1997), 207-260.

[TY] L. Tan and Y. Yin, Local connectivity of the Julia set for geometrically finite rational maps, Science in China Ser. A 39 (1996), 39-47.

Institute of Mathematics

Warsaw University

Banacha 2

02-097 Warszawa, Poland

E-mail: baranski@mimuw.edu.pl

Received 1 December 1997;

in revised form 30 April 1999 and 20 May 2000 